\title{
ANALISE DE COVARIÂNCIA EM DELINEAMENTOS DE BLOCOS COMPLETOS AUMENTADOS (BLOCOS DE FEDERER)
}

\author{
EDMILSON DE ARAUJO SILVA
}

Orientador: Prof. Dr. ROBERTO SIMIONATO MORAES

Dissertação apresentada à Escola Superior de Agricultura "Luiz de Queiroz", da Universidade de São Paulo, para obtenção do título de Mestre em Agronomia. Área de Concentração: Estatística e Exporimentação Agronômica.

\author{
$P I R A C I C A B A$ \\ Estado de São Paulo - Brasil \\ Junho - 1987
}


$\bar{A}$ Deus.

Aos meus pais José Mariano e Neusa Maria, aos meus irmãos, cunhados e sogros,

pelo carinho.

$\bar{A}$ minha querida esposa Maria Helena, aos meus filhos Guilherme e Giovanni, dedico. 


\section{AGRADECIMENTOS}

- Ao Dr.Roberto Simionato Moraes, Professor Assistente Doutor do Departamento de Matemática e Estatística da ESALQ/USP pe 1 a orientação.

- Aos Professores do Curso de Pós-Graduação em Estatística e Experimentação Agronōmica da ESALQ/USP, na pessoa de seu Coordenador Prof. Dr. Décio Barbin, pela acolhida e ensinamentos prestados.

- Aos colegas do Departamento de Estatística e Computação do Instituto de Ciências Exatas da Universidade do Amazonas pe 10 apoio recebido.

- A Universidade do Amazonas, a CAPES e ao CNPq pelo apoio fí nanceiro, sem o qual não seria possível a realização deste curso.

- Ao Prof. Dr. Cássio Roberto de Melo Godoi, pela revisão do Summary e auxîlio na elaboração do exemplo.

- Ao Prof. Dr. Lusbi Antonio Herreta Sandoval pelo incentivo e ajuda.

- Ao colega Carlos Tadeu dos Santos Dias pela colaboração na anālise do exemplo através do sistema computacional (Algema) de sua autoria. 
Aos funcionārios do Centro de Informātica na Agricultura (CIAGRI), pela atenção e ajuda prestada.

- A Sra. Isabel Cristina S. Andreucetti pelo excelente trabaTho datilogrāfico.

- Aos funcionārios do Departamento de Matemática e Estatística da ESALQ/USP pela colaboração. 
RESUMO $\ldots \ldots \ldots \ldots \ldots \ldots \ldots \ldots \ldots \ldots \ldots \ldots \ldots \ldots \ldots \ldots \ldots \ldots \ldots$

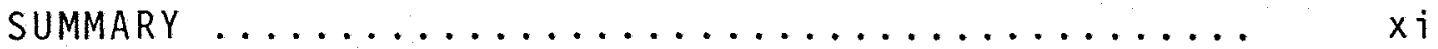

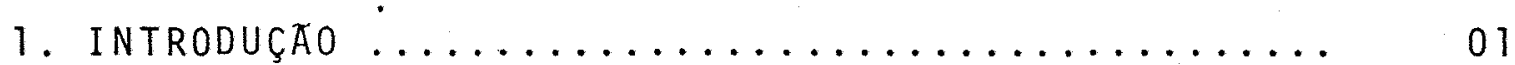

2. REVISAO BIBLIOGRAFICA ................. 03

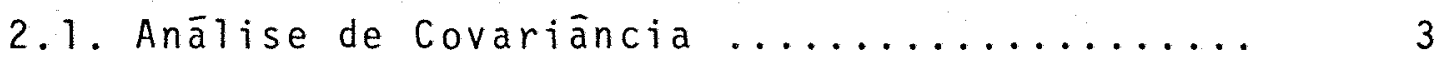

2.2. Ensaios em Blocos Completos Aumentados (Blocos de Federer) ................. 13

2.3. Anālise de Covariāncia em Delineamentos Quais quer com $p$ Variāveis Auxiliares ......... 17

3. DESENVOLVIMENTO TEORICO ............... 27

3.1. Ensaios em Blocos Completos Aumentados (Blocos de Federer) ................. 27

3.1.1. Definição dos parāmetros ........ 27

3.1.2. Um modelo linear ............ 27

3.1.3. Sistema de equações normais ........ 29

3.1.4. Estimação dos efeitos de tratamentos ajustados para blocos ........... 32

3.1 .4 .1 . Método clässico .......... 33

3.1.5. Somas de quadrados ............ 39

3.1 .6 . Esperanças matemāticas das somas de quadrados 38

3.1.6.1. Esperança matemática de $\underset{\sim}{Y} \underset{\sim}{Y} \ldots \ldots . .38$

3.1.6.2. Esperança matemática de $\underline{\sim}^{\prime} k^{-1} \underline{\sim} \ldots . . . .39$

3.1.6.3. Esperança matemática de $\tau_{0}{ }^{\prime} Q \ldots \ldots \quad 40$

3.1.6.4. Esperança matemātica da SQR ..... 41

3.1.7. Quadro da anāi ise de variância ..... 42

3.2. Anālise de Covariāncia em Blocos Completos Aumentados (Blocos de Federer) .......... 43 
Pāgina

3.2.1. Modelo matemático .......... 43

3.2.2. Sistema de equações normais .... 46

3.2.3. Estimação dos efeitos de tratamen tos ajustados para blocos e regressão.. 48

3.2.4. Somas de quadrados .......... 53

3.2.4.1. Soma de quadrados de paràmetros ........... 53

3.2.4.2. Soma de quadrados do resíduo, ajustada para rearessão $\ldots \ldots \ldots \ldots \ldots$

3.2.4.3. Soma de quadrados de tra tamentos, ajustáda para blocos e regressão .....

3.2.5. Esperanças matemāticas das somas de quadrados $\ldots \ldots \ldots \ldots \ldots$

3.2.5.1. Esperança matemática de ${ }_{\sim 0}^{0} Q \ldots \ldots \ldots \ldots \ldots \ldots$

3.2.5.2. Esperança matemática da SQReg $\ldots \ldots \ldots \ldots \ldots \ldots$.

3.2.5.3. Esperança matemātica de $\tilde{\gamma} \lambda_{2} \ldots \ldots \ldots \ldots \ldots \ldots$

3.2.5.4. Esperança matemātica da $\mathrm{SOT}_{\mathrm{aj}}^{*} \cdot \ldots \ldots \ldots \ldots \ldots$

3.2.5.5. Esperança matemātica de $\underline{Y^{\prime}} \underline{\underline{Y}} \ldots \ldots \ldots \ldots \ldots \ldots$ 
Pàgina

3.2.5.6. Esperança matemātica: de $\underline{B}^{\prime} K^{-1} \underset{\sim}{B} \ldots \ldots \ldots \ldots \ldots$

3.2.5.7. Esperança matemātica da $\mathrm{SQR}^{*} \ldots \ldots \ldots \ldots \ldots$

3.2.6. Distribuição das formas quadrāti-

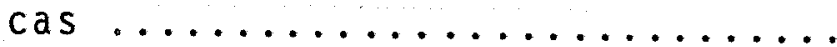

3.2.6.1. Distribuição de ${\underset{\sim}{0}}_{0}^{0} Q / \sigma^{2}$.

3.2.6.2. Distribuição

da SOReg $/ \sigma^{2}$

3.2.6.3. Distribuição

de $\tilde{\gamma} \lambda_{2} / \sigma^{2} \ldots \ldots \ldots \ldots$

3.2.6.4. Distribuição

da SOT S $j .^{*} / \sigma^{2} \ldots \ldots \ldots \ldots$

3.2.6.5. Distribuição

de $\underline{Y} Y \sigma^{2} \ldots \ldots \ldots \ldots \ldots$

3.2.6.6. Distribuição

de $\underset{\sim}{B} K^{-1} \underset{\sim}{B} / \sigma^{2} \ldots \ldots \ldots \ldots$

3.2.6.7. Distribuição

da $S Q R * / \sigma^{2} \ldots \ldots \ldots \ldots \ldots$

3.2.6.8. Distribuição do quociente $\frac{\mathrm{SQReg} \cdot / \mathrm{n}_{2}}{\mathrm{SQR}^{*} / \mathrm{n}_{7}}, \ldots \ldots \ldots$

3.2.6.9. Distribuição do quociente $\frac{S_{a T^{*}}^{*} / n_{4}}{S Q R^{*} / n_{7}} \ldots \ldots \ldots$ 
Pāgina

3.2.7. Estimadores das mēdias de tratamentos ajustados parablocos e regressão $\ldots \ldots \ldots \ldots \ldots \ldots \ldots$

3.2.8. Quadro da anālise de covariāncia 91

4. IlUSTRAÇAO DO METODO PROPOSTO $\ldots \ldots \ldots \ldots \ldots . . .9$ ?

5. CONCLUSOES ........................... 101

6. REFERENCIAS BIBLIOGRAFICAS .......... 103 


\section{ANALLISE DE COVARIÂNCIA DE DELINEAMENTOS EM BLOCOS \\ COMPLETOS AUMENTADOS (BLOCOS DE FEDERER)}

Autor: Edmilson de Araujo Silva

Orientador: Prof. Dr. Roberto Simionato Moraes

RESUMO

No presente trabalho considera-se uma variāvel auxiliar num ensaio em blocos completos aumentados (blocos de Federer). O objetivo foi apresentar um mētodo de anālise para esse tipo de ensaio.

Os parãmetros que caracterizam os delineamentos em blocos completos aumentados foram definidos como:

$c$ : nümero de tratamentos comuns,

b : nümero de blocos,

$z$ : número de tratamentos regulares,

$r_{j}$ : nümero de repetição do tratamento $j(j=1,2, \ldots, c, c+1, \ldots$, $c+z)$

$\mathrm{K}_{i}$ : nūmero de parcelas no bloco $i(i=1,2, \ldots, b)$

$N$ : nümero total de parcelas $(N=b c+z)$

o modelo matemático adotado foi o seguinte: 


$$
y_{i j}=\mu+\beta_{i}+t_{j}+\gamma x_{i j}+e_{i j},
$$

onde,

$y_{i j}$ e o valor observado da parcela do bloco $i$ que recebeu o tratamento $j$;

$\mu \quad \bar{e}$ a média geral;

$\beta_{i} \quad \bar{e}$ o efeito do bloco i;

$t_{j} \quad \bar{e}$ o efeito do tratamento $j$;

$\gamma \quad \bar{e}$ o coeficiente da regressão linear de $Y$ em relação a $X$ $x_{i j}=x_{i j}-\bar{x}$, onde $x_{i j}$ são os valores observados da variāvel auxiliar (covariāvel)

$e_{i j} \quad \bar{e}$ o erro experimental associado à observação $y_{i j}$, onde se supõe que os $e_{i j}$ 's são independentes e normalmente distri buĩdos, com média zero e variāncia $\sigma^{2}$.

0 efeito $t_{j}$ envolve $t_{s}(s=1,2, \ldots, c) \quad e$

$t_{r_{s^{\prime}}}\left(s^{\prime}=c+1, c+2, \ldots, c+z\right)$, que são os efeitos dos tratamen tos comuns e regulares, respectivamente.

Sob as condições anteriores são determinados: 0 sistema de equações normais, os estimadores dos efeitos dos parämetros, as somas de quadrados e suas esperanças matemáticas; e ainda, as distribuições das formas quadrāticas e um quadro da anālise de covariância.

Um exemplo numérico é apresentado para ilustrar o método proposto. 


\section{ANALYSIS OF COVARIANCE IN AUGMENTED RANDOMIZED \\ COMPLETE BLOCK DESIGN (BLOCK OF FEDERER)}

Author: Edmilson de Araujo Silva

Adviser: Prof. Dr. Roberto Simionato Moraes

SUMMARY

A statistical investigation was conducted for the case in which experiments are designed in augmented randomized complete block with a concomitant variable.

The main objetive was to develop a method of analysis for this specific kind of experiment.

The following parameters were considered as pertinent to the design:

$c$ : number of standard treatments;

b : number of blocks;

$z$ : number of new treatments;

$r_{j}$ : number of replicate in the $j^{\text {th }}$ treatment;

$k_{i}$ : number of plots in the $i^{\text {th }}$ block $(i=1,2, \ldots, b)$;

$N$ : total number of plots $(N=b c+z)$.

The following mathematical model was considered: 


$$
y_{i j}=\mu+\beta_{i}+t_{j}+\gamma x_{i j}+e_{i j},
$$

where:

$y_{i j}$ observed value due to $j^{\text {th }}$ treatment and $i^{\text {th }}$ block;

$\mu$ denotes the effect of the general mean;

$\beta_{i}$ denotes the effect of the $i^{\text {th }}$ block;

$t_{j}$ denotes the effect of the $j^{\text {th }}$ treatment;

$\gamma$ is a regression coefficient for the relation between $Y$ and $X$

$x_{i j}=x_{i j}-X$, where $x_{i j}$ is the centered observed value of the concomitant variable;

$e_{i j}$ is a random error component with expectation zero and variance $\sigma^{2}$, the $e_{i j}$ 's being independently distributed.

The effect $t_{j}$ involves $t_{s}(s=1,2, \ldots, c)$ and $t_{r_{s^{\prime}}}\left(s^{\prime}=c+1, c+2, \ldots, c+z\right)$, respectively the standard and new treatments.

Under the given conditions, the solution of the normal equations, the estimators of the parameters effects, the sum of squares, the expectations of sum of squares, the distributions of the quadratic forms and the analysis of covariance table are calculated.

A numerical example is used to illustrate the proposed method. 
1. INTRODUÇAO

o delineamento de blocos aumentados, introduzido por FEDERER (1956a) com o objetivo de permitir o uso da esta tîstica, atravēs da anāilise de variāncia, nas fases iniciais dos programas de melhoramento da cana-de-açūcar, foi de grande importāncia para os melhoristas.

Esse delineamento apresenta dois grupos de tratamentos:

1) Tratamentos comuns (c), repetidos b vezes;

2) Tratamentos regulares ( $z$ ), aparecendo uma ūn ca vez em todo experimento.

A aplicação de delineamentos em blocos completos aumentados na experimentação agrícola, apresenta a vantagem de comparar novas variedades, tratamentos, ou novas metodologias, 
quando a quantidade de material disponível é limitada. Por exem plo, em melhoramento de plantas há necessidade de testar um nümero muito grande de tratamentos (genōtipos), porēm a quantí dade de materia.t propagativo por genōtipo frequentemente è pé quena.

Entretanto, as añ̄ises de variāncia permitem o estudo da variāvel sem levar em conta provāveis influēncias de outras.

Esse fato e mais falta de repetição de alguns tratamentos pela exigüidade de material, razão da criação do delineamento, implicou em fazer um estudo da viabilidade do uso da anālise de covariāncia para este tipo de delineamento, jä que a covariância além de melhorar o controle local, bastante afetado pela falta de repetição, leva em consideração outras variāveis que podem influenciar naquela que estamos interessados.

No presente trabaitho é apresentado um estudo so bre a anālise de covariāncia para o delineamento em blocos com pletos aumentados quando a covariável é linear. A justificativa deve-se a escassez de informações na bibliografia e a impor tância desse delineamento no melhoramento da cana-de-açücar. 


\section{REVISAOO BIBLIOGRAFICA}

\subsection{Anālise de Covariância}

Segundo CoX e McCULLAGH (1982) a anāitise de covariância foi introduzida por Fisher, Conforme estes autores Eden e Fisher (1927) apresentaram a decomposição de uma soma de produtos e os correspondentes coeficientes de correlação. Sanders (1930) por sugestão de Fisher foi o primeiro a usar a anālise de covariāncia para aperfeiçoamento da precisão. Tal procedimento foi descrito por Fisher (1932) no livro "Statisti cal Methods for Research Workers", no qual aplicou-se o mëtodo da anālise de variancia usual a y - $\bar{\gamma} z$, onde y é a resposta, z é a covariāvel e $\bar{\gamma} \bar{e}$ a estimativa do coeficiente de regressão calculado via anālise de covariāncia. Esta foi admitida apenas como uma aproximação do teste $F$ aplicado aos tratamentos. Dois trabalhos mostrando aplicações da anālise de covariāncia foram apresentados na Royal Statistical Society (Wishart, 1934; Wits don, 1934). Fisher contribuiu na discussão de ambos e apre- 
ciou os pontos nos quais foi admitida a aplicação de um teste $F$ exato. Este fato foi muito discutido por Fisher (1935) no livro "Design of Experiments".

COCHRAN (1957) cita as principais finalidades do uso da anāitise de covariāncia, que são:

i) aumentar a precisão nos experimentos casualizados;

ii) remover efeitos de variāveis perturbadoras, em estudos estatisticos;

iii) contribuir para esclarecer a natureza dos efeitos de tratamentos;

iv) ajustar regressões em classificações mūitiplas;

v) analisar dados quando algumas observações são perdidas.

0 autor pondera que as hipóteses requeridas para o uso vālido da anālise de covariāncia, são uma extensão na tural daquelas requeridas para uma anălise de variância, a saber:

i) os efeitos de tratamentos, de blocos e da regressão devem ser aditivos;

ii) os residuos $\left(e_{i j}\right)$ devem ser independentes e normalmente distribuídos, com mëdia zero e mesma variāncia; 
0 autor recomenda que as precauções gerais que são tomadas para o uso da anālise de variāncia devem ser igua 1 mente observadas na anālise de covariância. Observa que, na anālise de covariāncia, se supõe que a forma correta da equa ção de regressão tenha sido ajustada.

Segundo o autor, os casos da anālise de cova riância com mais de uma variāvel auxiliar são denominados como covariância mūltipla. No caso de duas variāveis auxiliares, $x e z$, as estimativas dos coeficientes de regressão podem ser obtidas do sistema de equações:

$$
\begin{aligned}
& E_{x x} \hat{b}_{y \cdot x}+E_{x z} \hat{b}_{y \cdot z}=E_{x y} \\
& E_{x z} \hat{b}_{y \cdot x}+E_{z z} \tilde{b}_{y \cdot z}=E_{z y},
\end{aligned}
$$

onde $E_{x x}, E_{z z}, E_{x z}, E_{x y}$ e $E_{z y}$ são as somas de quadrados do re sỉduo relativas às variāveis $x, z, x z, x y$ e $z y$, respectivamente.

A estimativa da variāncia da diferença entre dois efeitos de tratamentos é dada, por:

$$
s_{e}^{2}\left[\begin{array}{l}
\frac{2}{r}+\left(x_{i .}-x_{j \cdot}\right)^{2} c_{x x^{+}} \\
2\left(x_{i .}-x_{j .}\right)\left(z_{i .}-z_{j .}\right) c_{x z^{+}} \\
+\left(z_{i}-z_{j .}\right)^{2} c_{z z}
\end{array}\right]
$$

e, a estimativa da variancia mēdia, sobre todos os pares de tratamentos, por: 
$\frac{2}{r} s_{e}^{2}\left(1+t_{x x} c_{x x}+2 t_{x z} c_{x z}+t_{z z} c_{z z}\right)$,

onde:

$t_{x x}, t_{z z}$ e $t_{x z}$ são os quadrados médios de tratamentos relativos às variāveis $x, z$ e $x z$, respectivamente;

$c_{x x}, c_{z z}$ e $c_{x z}$ são os elementos da inversa da matriz contituida pelas somas de quadrados $E_{x x}, E_{z z}$ e $E_{x z}$;

$x_{i}$ e $z_{i}$ são as médias do $i$-ésimo tratamento relativas às variāveis $x$ e $z$, respectivamente;

$\mathrm{s}_{\mathrm{e}}^{2} \quad \overline{\mathrm{e}}$ o quadrado médio do resíduo, ajustado para regressão;

$r \quad \bar{e}$ o nümero de repetições.

KIRK (1968) destaca as vantagens e desvantagens da anālise de covariāncia. As maiores vantagens da anāilise de covariāncia apresentadas pelo autor são:

i) Permite um experimentador remover uma ou mais fontes potenciais de viés que são difíceis de eliminar através de um controle expe rimental;

ii) A anālise de covariância dá aproximadamente a mesma redução no erro experimental que o uso de delineamentos em blocos casualizados. Ela pode ser usada depois que os dados tenham sido coletados. A estratificação dos 
individuos em blocos homogêneos precisa ser feito no começo do experimento;

As maiores desvantagens da anālise de covariāncia, segundo o autor, são:

i) Requer cálculos mais laboriosos que o correspondente na anālise de variāncia;

ii) A anālise de covariāncia requer um conjunto de restrições de suposições que podem tornar impraticāvel o seu uso num determina do ensaio;

iii) Förmulas computacionais para estimar observações perdidas e fazer comparações entre médias para algum delineamento da anālise de covariāncia são relativamente complexos.

NETER e WASSERMAN (1974) ressaltam as idéias fun damentais para o uso de modelos de covariāncia em delineamentos inteiramente casualizados e blocos casualizados. Discutem os problemas dos modelos de covariância na determinação das regressões. Finalmente indicam como a anālise de covariāncia pode ser considerada como uma modificação da añāise de variância.

Asseguram os autores que nos delineamentos inteiramente casualizados os erros experimentais podem ser devi- 
dos as heterogeneidades das unidades experimentais. Uma poss bilidade para reduzir os erros experimentais sem utilizar os delineamentos em blocos é empregar a anālise de covariāncia. Nos casos em que o delineamento em hlocos não é viāvel então a anālise de covariancia pode ser usada para reduzir o erro experimental.

Segundo os autores, na terminologia da anālise de covariância a variável quantitativa independente é chamada de variāvel concomitante onde a escolha dessa variāvel é muito importante. Se a variāvel concomitante não tem relação com a variāvel resposta nada se ganha com o uso da anālise de cova riāncia. Quanto a escolha da variāvel concomitante ela não pode ser influenciada pelas variāveis qualitativas (tratamentos). E mais usual, porēm, não é recra geral, usar como variävel conco mitante, uma variāvel obtida antes da instalação do experimento, sorteando-se os tratamentos independentemente dos valores da variável concomitante.

Os autores discutem a comparação de tratamentos para os casos em que as retas de regressão estimadas para tratamentos são paralelas e pará os casos em que não são.

Para os autores a linearidade da relação entre Y e $X$ não é essencial na anālise de covariāncia. Qualquer outra relação pode ser usada, como a quadrática, cujo modelo fica: 
$y_{i j}=\mu+\tau_{j}+\gamma_{1}\left(x_{i j}-\bar{x} \ldots\right)+\gamma_{2}\left(x_{i j}-\bar{x} \ldots\right)^{2}+e_{i j}$

Entretanto, quase sempre o modelo linear é uma boa aproximação.

Os testes de adequacidade do modelo de covariàn cia linear para os autores, exigem alguns fatos, ou seja:

1) Normalidade dos erros;

2) Homogeneidade de variâncias;

3) Igualdade dos coeficientes de regressão para as equações dos tratamentos;

4) Linearidade da regressão.

Os autores tambēm apresentam o seguinte procedi mento de formulação da regressão.

Para estimar os paràmetros do modelo de covariàn cia $Y_{i j}=\mu .+\tau_{j}+\gamma\left(X_{i j}-\bar{X}\right)+\varepsilon_{i j}$ (I) e analisar questões de interesse utilizamos o procedimento por regressão.

Denotando por $z_{i j}=\left(x_{i j}-\bar{x}\right)$ no modelo de cova riância (I) e usando $r-1$ variāveis indicadoras para os $r^{\prime} s$ tra tamentos, tem-se:

$$
x_{1}=\left\{\begin{array}{l}
1 \text { se a observação for do tratamento } 1 \\
0 \text { caso conträrio }
\end{array}\right.
$$




$$
x_{r-1}=\left\{\begin{array}{l}
1 \text { se a observação for do tratamento } r-1 \\
0 \text { caso contrārio }
\end{array}\right.
$$

Então, podemos expressar o modelo de covariancia

(I) como segue:

$Y_{i j}=\beta_{0}+\beta_{1} X_{i j 1}+\ldots+\beta_{r-1} X_{i j r-1}+\beta_{r} Z_{i j}+\varepsilon_{i j}$

As correspondências entre os parâmetros nos modelos de regressão (II) e os do modelo da anālise de covariância (I) são os seguintes:

$$
\begin{aligned}
& B_{0}=\mu .+\tau_{r} \\
& \text { (III) } \quad \beta_{j}=\tau_{j}-\tau_{r} \quad j=1, \ldots, r-1 \\
& { }^{\beta} r=\gamma
\end{aligned}
$$

Estas correspondencias são estabelecidas na for ma usual. Por exemplo, se uma unidade experimental recebeu $r$ tratamentos, o modelo da anālise de covariância (I) produz:

$\gamma_{i r}=\mu_{0}+\tau_{r}+\gamma z_{i r}+\varepsilon_{i r}$

Com o modelo de regressão (II), as variāveis $x$ tem os valores:

$x_{1}=0 \quad x_{2}=0 \ldots x_{r-1}=0$. Logo, obtemos o modelo de re gressão: 
$Y_{i r}=\beta_{0}+\beta_{r^{Z}}{ }_{i r}+\varepsilon_{i r}$

Igualando (IV) e (V), tem-se:

$\beta_{0}=\mu_{0}+\tau_{r}$

${ }^{B} r=\gamma$. As outras correspondências são obtidas similarmente.

E evidente que através de (III) os testes concer nentes aos efeitos de tratamentos $\tau_{j}$ podem ser feitos da mesma maneira que sobre os coeficientes de regressão $\beta_{j}(j=1, \ldots, r-1)$. Similarmente, estimativas dos efeitos de tratamentos podem ser obtidas, em termos de estimativas dos coeficientes de regressão.

os autores através de um exemplo desenvolvem um modelo, estimam e testam os efeitos de tratamentos e fazem um teste de paralelismo. Em seguida, apresentam a anālise de covariāncia como uma modificação da anālise de variāncia e em ex perimentos fatoriais.

Os autores tecem considerações sobre o uso da anālise de variância em casos de tomar-se diferenças de variāveis, tambēm chamadas de indices de respostas para anālise. Estas levam segundo os autores aos mesmos resultados das análises de covariancia das duas variāveis, reduzindo consideravelmente o trabalho.

Mostram que, se a inclinação da reta de regres- 
são é igual a 1 , as anālises de variância e covariāncia são absolutamente equivalentes, pois o modelo de covariancia fica $\dot{y}_{i j}=\mu \cdot+\tau_{j}+x_{i j}+\varepsilon_{i j}$ que pode ser escrito como um modelo normal de anālise de variáncia da seguinte maneira:

$$
y_{i j}-x_{i j}=\mu \cdot+\tau_{j}+\varepsilon_{i j} .
$$

PIMENTEL GOMES (1985), para a anālise de covariância nos delineamentos em blocos completos casualizados e com apenas uma variāvel auxiliar estabelece, para o cálculo da soma de quadrados de tratamentos, ajustada para regressão, o seguinte procedimento:

$$
S Q T^{*}=R^{\prime}\left(y^{2}\right)-\frac{\left[R^{\prime}(x y)\right]^{2}}{R^{\prime}\left(x^{2}\right)}-S Q \operatorname{Res} *
$$

onde:

$$
\begin{aligned}
& R^{\prime}\left(y^{2}\right)=R\left(y^{2}\right)+\operatorname{SQT}(y) ; \\
& R^{\prime}\left(x^{2}\right)=R\left(x^{2}\right)+\operatorname{SQT}(x) ; \\
& R^{\prime}(x y)=R(x y)+\operatorname{SPT}
\end{aligned}
$$

$R\left(y^{2}\right)$ e $\operatorname{SQT}(Y)$ são as somas de quadrados do resíduo e de tratamentos, relativas à variāvel dependente $Y$, respectivamente;

$R\left(x^{2}\right)$ e $\operatorname{SRT}(X)$ são as somas de quadrados do resíduo e de tratamentos, relativas à variável auxiliar $x$, respectivamente; 
$R(x y)$ e SPT são as somas de produtos da variāvel dependente Y com a variável auxiliar $X$, relativas ao resíduo e a tratamentos, respectivamente;

SQ Res* é a somá de quadrados do residuo, ajustada para regres são.

0 autor testa o coeficiente de correlação linear, pelo teste $t$, através da estatística:

$$
t=\frac{r}{\sqrt{1-r^{2}}} \sqrt{n_{e}}
$$

com $n_{e}$ graus de liberdade, onde:

$r=\frac{R(x y)}{\sqrt{R\left(x^{2}\right) R\left(y^{2}\right)}}$ é a estimativa do coeficiente de correlação

ne é o número de graus de liberdade do resīduo, depois de ajustado para regressão. Observa que a anālise de covariância pode ser feita independentemente da significāncia do valor de $r$ obtido.

Segundo o autor, o procedimento de utilizar-se uma estimativa média para a variāncia da diferença entre duas mēdias de tratamentos, ajustadas para regressão, sō deve ser utilizado quando a variável auxiliar não interfere nos trata mentos. 


\subsection{Ensaios em Blocos Completos Aumentados (Blocos de Federer)}

Devido a necessidade de um delineamento experimental mais eficiente na comparação de plāntulas, ("seedlings") a variedade ("clones), nas fases iniciais de um programa de melhoramento de cana-de-açūcar, abacaxi, etc. FEDERER (1956a, 1956b, 1961) desenvolveu uma metodologia que permite comparar estas novas variedades, chamadas de variedades regulares, com variedades jā existentes, ou comerciāveis, chamadas de comuns; chamando este novo delineamento de "Delineamento aumentado", que é um tipo de delineamento experimental, formado a partir dos delineamentos inteiramente ao acaso, blocos casualizados e quadrado latino.

Estes delineamentos apresentam dois grupos de tratamentos, os comuns, repetidos b vezes, e um segundo grupo, os regulares, que aparecem uma única vez no experimento.

o delineamento em blocos completos aumentados proposto pelo autor permite uma estimativa do erro eperimental, com a vantagem de permitir a inclusão de um grande número de tratamentos utilizando um numero relativamente pequeno de parcelas. Este consiste em um delineamento clássico em blocos ao acaso sendo que em cada bloco pode-se agregar um certo nūmero de novos tratamentos que aparecem uma ūnica vez no delineamento. 0 nümero de tratamentos por bloco não preci sa ser igual o que dá maior flexibilidade ao pesquisador.

MARTINEZ GARZA (1972), apresenta o seguinte procedimen 
to de casualização para o delineamento em blocos completos aumentados.

A casualização è feita levando em conta que hã b blocos com $k_{i}$ parcelas $(i=1,2, \ldots, b)$. Inicialmente, sorteiam-se os $c$ tratamentos comuns a c das $k_{i}$ parcelas de cada bloco, em seguida, sorteiam-se os z tratamentos regulares entre as parcelas restantes de cada bloco.

NOGUEIRA (1976) estuda a anālise conjunta de en saios em blocos casualizados completos aumentados, cada ensaio apresenta os mesmos $t=c+z$ tratamentos, distribuidos em $r$ blocos, onde os c tratamentos são considerados comuns pois apa recem nos $r$ blocos, e os $z$ tratamentos são considerados regula res, pois aparecem uma ünica vez em um dos $r$ blocos. Os blocos são formados por $k$ parcelas, sendo $k=c+p_{j}$, onde: $p_{j}(j=1,2, \ldots, r) \bar{e}$ o número de tratamentos reguiares por b 1 oco.

Para desenvolvê-1o, considerou o conjunto dos ensaios como um delineamento comum em blocos incompletos, baseado nos trabalhos de Pimentel Gomes e Guimarães (1958), Pavete (1961) e Pimentel Gomes (1970), admitindo que os ensaios apresentassem variâncias semelhantes.

VIZONI (1984) faz um estudo do delineamento em blocos aumentados com parcelas subdivididas no tempo, tendo 
por finalidade, principalmente a aplicação em experimentos agronômicos, aproveitando as produções de anos sucessivos.

$0^{*}$ autor considerou, c variedades comans dispostas em b blocos, e $z$ variedades regulares as quais aparecem uma ūni ca vez em todo o experimento e v anos. E ainda que todos os blocos tenham o mesmo número de parcelas $(k)$, nada impedindo entretanto que esses nümeros sejam diferentes.

Adotou o seguinte modelo matemātico:

$y_{1 j v}=m+t_{1}+b_{j}+(t b)_{1 j}+a_{v}+t a_{1 v}+b a_{j v}+e_{1 j v}$.

Quando se trabalha com parcelas subdivididas no tempo, não è raro encontrar correlações diferentes entre duas subparcelas de uma mesma parcela. Neste estudo, por ser um tra balho inicial, o autor considerou apenas o caso onde estas correlações são constantes e existe a independencia entre subparcelas de parcelas distintas, resultando:

$\operatorname{cov}\left(y_{1 j v^{\prime}}, y_{l^{\prime} j^{\prime} v^{\prime}}\right)= \begin{cases}\sigma^{2} & \text { se } 1=1^{\prime} ; j=j^{\prime} ; v=v^{\prime} \\ \sigma^{2} & \text { se } 1=1^{\prime} ; j=j^{\prime} ; v \neq v^{\prime} \\ 0 & \text { em outros casos }\end{cases}$

Nestas condições, baseando-se na metodologia apresentada por Pimentel Gomes (1967), para blocos incompletos balanceados, e no trabalho desenvolvido por Iemma (1981), so- 
bre parcelas subdivididas, o autor determinou: o sistema de equações normais, somas de quadrados dos parāmetros, as matrizes de dispersão, e as comparações mūltiolas pelo critērio de Tu key.

2.3. Anāilise de Covariäncia em Delineamentos Quaisquer com p Variāveis Auxiliares.

KATTI (1965) estudando anālise de covariāncia em qualquer tipo de delineamento, com p variáveis auxiliares, utiliza o seguinte modelo matemático:

$$
\underline{y}=x_{\underline{B}}+\underline{z} \underline{\underline{b}}+\underline{\mathrm{e}}
$$

onde:

$\underline{Y}$ ē o vetor das $n$ observações da variāvel dependente;

$x$ é a matriz dos coeficientes do delineamento de dim $n \times q$;

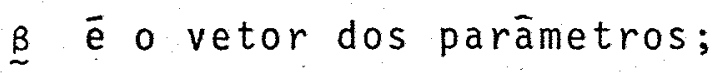

$Z$ é a matriz dos valores observados das p variáveis auxiliares;

$\stackrel{\text { b }}{\text { e }}$ o vetor dos coeficientes de regressão;

e $\bar{e}$ o vetor dos erros experimentais, onde $\underset{\sim}{e} \sim N\left(\theta, \sigma^{2} I\right)$.

Para obter as soluções, o autor reescreveu 0 modelo (2.3.a) da seguinte maneira:

$$
\begin{aligned}
\underline{Y} & =x(\underline{B}+\Delta \underline{\sim})+\left(Z-x_{\Delta}\right) \underline{b}+\underline{e} \\
& =x_{\underline{B}}^{*}+\left(Z-x_{\Delta}\right) \underline{b}+\underline{e}
\end{aligned}
$$

onde $\Delta$ deve satisfazer 0 sistema $X^{\prime}(Z-X \Delta)=\emptyset$. 
Pondera o autor que, se $X^{\prime} X$ é não singular, então $\bar{\Delta}=\left(X^{\prime} X\right)^{-1} X^{\prime} Z$. No caso geral, entretanto, $\bar{\Delta}=\left(X^{\prime} X\right)^{-} X^{\prime} Z$, é uma solução do sistema $X^{\prime}\left(Z-X_{\Delta}\right)=\emptyset$.

As estimativas obtidas através deste procedimen to são:

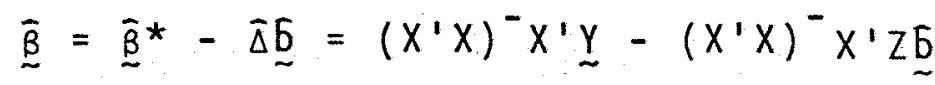

$\underset{\sim}{\underline{b}}=R_{1}^{-1} R_{2}=\left[(Z-X \widehat{\Delta})^{\prime}(Z-X \widehat{\Delta})\right]^{-1}\left[(Z-X \widehat{\Delta})^{\prime} \underline{Y}\right]$

onde:

$\overline{\text { }}^{*}$ e o vetor das estimativas dos parâmetros, obtido do modelo $Y=X \underline{B}^{*}+e ;$

$\left(X^{\prime} X\right)^{-\bar{e}}$ uma inversa generalizada da matriz $X^{\prime} X$;

$R_{1}^{-1} \bar{e}$ a inversa da matriz constituída pelas somas de quadrados e de produtos do resíduo, relativas às variāveis auxiliares;

$R_{2}$ é o vetor constituīdo pelas somas de produtos das variāveis auxiliares com a variāvel dependente, relativo ao resíduo.

Segundo esse autor, a matriz de variancias e covariancias de $\underset{\sim}{b} \bar{e}$ dada por:

$$
\begin{aligned}
& \operatorname{Var}(\underset{\sim}{b})=R_{1}^{-1} \sigma^{2} \text { e de } \underset{\sim}{\bar{B}} \overline{\mathrm{e}}: \\
& \operatorname{Var}(\underset{\sim}{\bar{B}})=\operatorname{Var}\left(\underline{\sim}^{*}\right)+\bar{\Delta} \operatorname{Var}(\underset{\sim}{b}) \hat{\Delta}^{\prime},
\end{aligned}
$$

onde:

$\operatorname{Var}\left(\hat{B}^{*}\right)=\left(X^{\prime} X\right)^{-} \sigma^{2}$ 
PIMENTEL GOMES (1967), estudando experimentos em blocos incompletos, atravēs de matrizes, eliminou inicialmente os efeitos de blocos, os quais considerou, para simplificar, como incluindo o efeito da média geral teórica. Assim procedendo, obteve o sistema $C \bar{T}=\underline{Q}$, onde a matriz $C \bar{e}$ singular. As so luções desse sistema foram obtidas introduzindo restrições, resultando no sistema, $A \widehat{T}=\emptyset$. Observou que a matriz $M=(C-A) \bar{e}$,por hipōtese, não singular, e que serā sempre simētrica se for utilizado $A_{1} \bar{T}=\emptyset$, onde $A_{1}=A^{\prime} A$.

SEARLE (1971) afirma que a anālise de covariāncia pode ser feita utilizando-se o modelo $\underset{\sim}{Y}=\underset{\sim}{X}+\underset{\sim}{e}$, onde $X$ é uma matriz composta de valores 0 e 1 e dos valores observados das covariāveis.

No entanto, para uma melhor distinção entre os dois tipos de parâmetros, o mesmo autor particionou adequadamente a matriz $x$ e o vetor $\underset{\sim}{b}$ em duas partes. o modelo, dessa forma, fica:

$$
\underline{Y}=\mathrm{X}_{\sim}+\underset{\sim}{\mathrm{zb}}+\underset{\sim}{\mathrm{e}} \text {, onde: }
$$

Y é o vetor das n observações da variāvel dependente;

$X$ representa a matriz de incidencia dos niveis dos fatores e interações, de rank = k;

$Z$ representa a matriz das covariáveis, suposta de posto coluna completo, cujas colunas são linearmente independentes das colunas de $\chi$; 
$\underset{\sim}{\text { e }}$ o vetor de parámetros correspondente a $\mu$, aos níveis dos fatores e às interações;

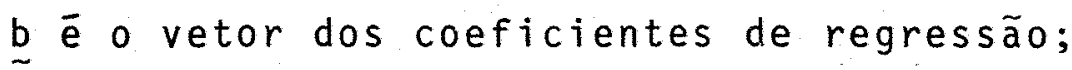

e $\bar{e}$ o vetor dos erros experimentais, e $\sim N\left(\emptyset, I \sigma^{2}\right)$

As soluções de mĩnimos quadrados são:

$$
\stackrel{\sim}{a}^{0}={\underset{\sim}{a}}^{*}-\left(X^{\prime} x\right)^{-} x^{\prime} \underline{\sim}^{0}
$$

e

$$
\underline{b}^{0}=\underline{b}=\left(Z^{\prime} P Z\right)^{-1} Z^{\prime} P \underline{Y}
$$

onde;

$\underline{\sim}^{*}=\left(X^{\prime} X\right)^{-} X^{\prime} \underline{\sim} ;$

$P=I-X\left(X^{\prime} X\right)^{-} X^{\prime}$;

$\left(X^{\prime} X\right)^{-} \bar{e}$ uma inversa generalizada, simétrica, de $X^{\prime} X$;

Z'PZ è a matriz constituída pelas somas de quadrados e de produtos do residuo, relativas às variāveis auxiliares; Z'PY é o vetor constituído pelas somas de produtos das variāveis auxiliares com a variāvel dependente, relativas ao residuo:

O autor mostra que $\underset{-6}{\mathrm{e}} \mathrm{o}$ melhor estimador $1 \mathrm{i}$ near não tendencioso ("blue") de $\underset{\sim}{b}$ nos modelos:

$\underline{y}=\underset{\sim}{x a}+z \underline{b}+\underline{e} \quad$ e $\quad \underline{y}=P \underset{\sim}{b}+\underline{e}$

Segundo o autor, no modelo $\underline{Y}=X \underset{\sim}{a}+\underset{\text { e, }}{0}$ vetor das estimativas dos valores esperados $\hat{y}$ correspondente ao vetor dos 
valores observados $Y \bar{e} \bar{Y}=X\left(X^{\prime} X\right)^{-} X^{\prime} \underline{Y}$. Portanto, o vetor dos desvios dos valores observados e os valores estimados é

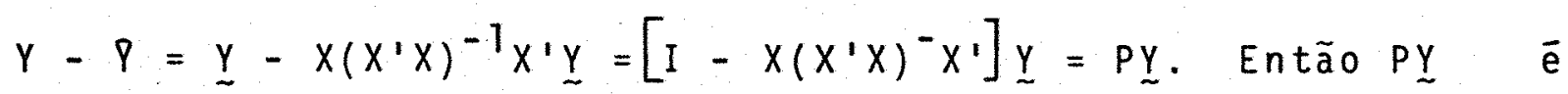
o vetor de resíduos após ajustar $\underset{\sim}{Y}=X_{\sim}+\underset{\sim}{e}$.

Similarmente, se a j-ésima coluna de $Z \bar{e} \underset{\sim}{z}$, a j-ésima coluna de $P Z$ em $\underset{\sim}{Y}=P Z \underset{\sim}{b}+\underset{\sim}{e} \bar{e} P \underset{\sim}{Z}$ e é o vetor de resīduos apōs ajustar $\underset{\sim}{z}=X_{\sim}+\underset{\sim}{e}$. Então com $Z=\left\{z_{j}\right\} \quad$ com $j=1,2, \ldots, q$ escreve-se $R_{z}$ por $P Z$ e tem-se $R_{z}$ como a matriz de resîduos.

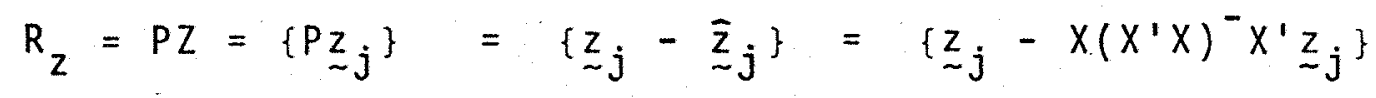

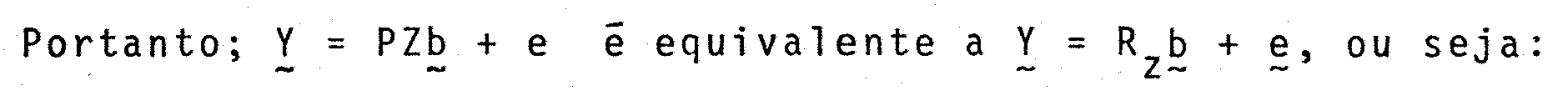

$$
E=\left(R_{Z}^{\prime} R_{Z}\right)^{-1} R_{Z}^{\prime} \underline{Y} \text { onde: }
$$

$R_{z}$ é a matriz de mesma ordem que $Z$, com suas colunas expressan do resíduos;

$R_{z}^{\prime} R_{z}$ e a matriz da soma de quadrados e produtos, dos residuos z;

$R_{Z}^{\prime} \underset{\sim}{Y} \bar{e}$ um vetor da soma de produtos dos residuos z pelas observações Y.

Dessa forma, o vetor solução para o modelo de covariância, é, então:

$$
\left[\begin{array}{l}
\stackrel{a}{a}^{0} \\
\underset{b}{b}
\end{array}\right]=\left[\begin{array}{l}
\stackrel{a}{*}^{*}\left(X^{\prime} X\right)^{-} X^{\prime} Z \underset{\sim}{b} \\
\left(R_{Z}^{\prime} R_{Z}\right)^{-1} R_{Z}^{\prime} \underline{Y}
\end{array}\right]
$$


As variāncias e a covariāncia entre os estimado res são dadas por:

$\operatorname{var}\left(\stackrel{\sim}{a}^{0}\right)=\left[\left(x^{\prime} x\right)^{-}+\left(x^{\prime} x\right)^{-} x^{\prime} z\left(R_{z}^{\prime} R_{z}\right)^{-1} z^{\prime} x\left(x^{\prime} x\right)^{-}\right]_{\sigma}{ }^{2}$

$\operatorname{var}(\underset{\sim}{\operatorname{b}})=\left(R_{z}^{\prime} R_{z}\right)^{-1} \gamma^{2}$

$\operatorname{cov}\left(\underline{a}^{0}, \underset{\sim}{b}\right)=-\left(x^{\prime} x\right)^{-} x^{\prime} z\left(R_{z}^{\prime} R_{z}\right)^{-1} \sigma^{2}$

0 autor apresenta os seguintes resultados para

as expressões $R(\underset{\sim}{a})$ e $R(\underset{\sim}{b} / \underset{\sim}{a})$ :

$$
\begin{aligned}
& R(\underline{a})=a^{*} X^{\prime} \underline{Y}=\underline{Y}^{\prime} X\left(X^{\prime} X\right)^{-} X^{\prime} \underline{\sim} \\
& a X^{\prime} \underline{\sim} / \sigma^{2} \sim X^{2}\left[r(X), \lambda_{a}\right] \text {, onde } \\
& \lambda_{a}=\frac{1}{2 \sigma^{2}}\left[\stackrel{a}{a}^{\prime} x^{\prime} x_{\sim}^{a}+2 \underline{\sim}^{\prime} x^{\prime} Z \underset{\sim}{b}+\underline{b}^{\prime} Z^{\prime} X\left(X^{\prime} X\right)^{-} x^{\prime} Z \underset{\sim}{b}\right] \\
& R(\underset{\sim}{b} / \underset{\sim}{a})=R(\underset{\sim}{a}, \underset{\sim}{b})-R(\underset{\sim}{a})=\underline{\sim}^{\prime} R_{z}\left(R_{z}^{\prime} R_{z}\right)^{-1} R_{z}^{\prime} \underset{\sim}{Y} \\
& =\underline{5}^{\prime} R_{Z} \underline{Y}
\end{aligned}
$$$$
B^{\prime} R_{Z}^{\prime} Y / \sigma^{2} \sim x^{2}[r(Z), \lambda] \quad \text { onde }
$$$$
\lambda=\frac{1}{2} \underline{b}^{\prime} R_{z}^{\prime} R_{z} \underline{b} / \sigma^{2} \text { e } r(z)=p \text {. }
$$

Alēm disso $R(\underset{\sim}{a})$ e $R(\underset{b}{b} / \underset{a}{a})$ são distribuidas independentemente porque $X\left(X^{\prime} X\right)^{-} X^{\prime} R_{z}\left(R_{z}^{\prime} R_{z}\right)^{-1} R_{z}=0$, visto que $R_{Z}=P Z$ e $X^{\prime} P=0$, onde $P=I-X\left(X^{\prime} X\right)^{-} X^{\prime}$ então $R(\underset{\sim}{a})$ e $R(\underset{\sim}{b} / \underset{\sim}{a})$ são independentes de SSE $=\left(\underline{y}^{\prime} \underline{y}-a^{*} X^{\prime} \underset{\sim}{y}\right)-\underset{\sim}{5} R_{z}^{\prime} \underline{y}$ que ē a soma de quadrados do residuo, ajustada para regressão e tem dis tribuição $x^{2}$ : 
$\frac{S S E}{\sigma^{2}} \sim x_{N}^{2}-r(X)-r(Z)$ onde

$N$ é o número de observações e $r(X)=k$.

A seguir, o autor, apresenta dois quadros da ana lise de variāncia, a saber: um para ajustar covariáveis (b) após fatores e interações (a) no modelo de covariāncia $\underset{\sim}{\gamma}=\underset{\sim}{x}+\underset{\sim}{b}+\underset{\sim}{b}$ e outro para ajustar fatores e interações (a) apōs covariāveis (b) nesse modelo.

0 autor mostra, ainda, que as hipóteses $H_{1}: \underset{\sim}{b}=0$, $\mathrm{H}_{2}: \mathrm{k}^{\prime} \underset{\sim}{\mathrm{a}}=\underset{\sim}{0} \mathrm{e} \mathrm{H}_{3}: \mathrm{k}^{\prime} \underset{\sim}{\mathrm{a}}+\left(\mathrm{X}^{\prime} \mathrm{X}\right)^{-} \mathrm{X}^{\prime} Z \underset{\sim}{\mathrm{b}}=\underset{\sim}{0}$, são testadas, respectivamente, por:

$$
\begin{aligned}
& F\left(H_{1}\right)=\frac{\sigma^{\prime} R_{Z}^{\prime} Y / r(Z)}{\operatorname{SSE} / N-r(Z)-r(X)} \\
& F\left(H_{2}\right)=\frac{Q / r(X)}{\operatorname{SSE} / N-r(Z)-r(X)} \\
& F\left(H_{3}\right)=\frac{Q * / r(X)}{\operatorname{SSE} / N-r(Z)-r(X)}
\end{aligned}
$$

sendo,

$$
\begin{aligned}
& Q=\left(k^{\prime} \stackrel{\sim}{a}^{0}\right)^{\prime}\left[k^{\prime}\left(x^{\prime} x\right)^{-k}+k^{\prime}\left(X^{\prime} x\right)^{-} x^{\prime} Z\left(Z^{\prime} P Z\right)^{-1} Z^{\prime} x\left(x^{\prime} x\right)^{-} k\right]^{-1} k^{\prime} \underline{a}^{0} \\
& \text { e } Q^{*}=\left(k^{\prime} \underline{\sim}^{*}\right)^{\prime}\left[k^{\prime}\left(X^{\prime} x\right)^{-} k\right]^{-1} k^{\prime} \underline{a}^{*}
\end{aligned}
$$

DIAS (1981), estudando a anālise de covariância 
intrablocos, com p variāveis auxiliares, no delineamento em biocos incompletos, caso particular dos ensaios equilibrados, considera o modelo matemático

$$
y_{i j}=\mu+t_{i}+b_{j}+\sum_{w=1}^{p} a_{w} x_{i j}^{(w)}+e_{i j},
$$

e admitindo que os efeitos de blocos $\left(b_{j}\right)$ incluem a média geral teórica $(\mu)$, apresenta os seguintes resultados:
a) Estimativa dos efeitos de tratamentos ajus- tados para blocos e regressão$$
\bar{t}_{i}=\bar{t}_{i} .-\sum_{w=1}^{p} \bar{a}_{w} \hat{t}_{i w} \quad(i=1,2, \ldots, v) \text {, }
$$

onde:

$\bar{t}_{i} \bar{e}$ a estimativa do efeito do i-ésimo tratamento ajustado para blocos e regressão;

$\bar{t}_{i \text {. }} \bar{e}$ a estimativa do efeito do i-ésimo tratamento ajustado para blocos relativo à variāvel y;

$\hat{t}_{i w}$ semelhante $\bar{a} \hat{t}_{i}$, porēm em relação à variável auxiliar $x^{(w)}$;

$\hat{a}_{w} \bar{e}$ a estimativa do coeficiente da regressão linear mūltipla, associado à variāvel auxiliar $x^{(w)}$.

b) Estimativa dos coeficientes de regressão

$$
\bar{a}=E_{3}^{-1} \cdot E_{4} \quad \text { onde }
$$

$E_{3}$ é uma matriz não singular, constituĩda pelas somas de quadrados e de produtos dos residuos, relativas às variāveis auxiliares;

$E_{4} \bar{e}$ o vetor contituido pelas somas de produtos das variāveis rauxiliares com a variável dependente, relativas ao resíduo. 
C) Soma de quadrados da regressão

SQ Reg. $=\underline{a}^{\prime} E_{4}=\sum_{w=1}^{p} \hat{a}_{w} R\left(x_{w} y\right)$

d) Soma de quadrados do residuo, ajustada para regressão.

SQ Res*. $=R\left(y^{2}\right)-\sum_{w=1}^{p} \hat{a}_{w} R\left(x_{w} y\right)$

onde $R\left(y^{2}\right)$ é a soma de quadrados do residuo, sem serem conside radas as variáveis auxiliares.

e) Soma de quadrados de tratamentos, ajustada para blocos e regressão.

$S Q T^{*}(a j)=R^{\prime}\left(y^{2}\right)-S Q \operatorname{Res} *-\sum_{w=1}^{p} \tilde{a}_{w^{\prime}} R^{\prime}\left(x_{w} y\right)$, onde $R^{\prime}\left(y^{2}\right)=\operatorname{SQT}\left(a j_{\cdot}\right)+R\left(y^{2}\right)$.

f) Esperança matemātica da SQ Reg. $E(S Q R e g)=.a^{\prime} E_{3} a+p \sigma^{2}$, justificando-se, assim, a associação de p graus de liberdade à soma de quadrados da regressão.

g) Esperança matemātica da SQ Res*. $E\left(S Q \operatorname{Res}^{*}.\right)=(b k-b-v-p+1) a^{2}$, justificando-se, assim, a associação de (bk - b - v - p + 1) graus de liberdade à soma de qua drados do resíduo, ajustada para regressão.

Portanto, um estimador imparcial de $\sigma^{2} \bar{e}$ : 


$$
\bar{\sigma}^{2}=\frac{S Q \operatorname{Res}{ }^{\star}}{(b k-b-v-p+1)}=Q M \operatorname{Res}{ }^{\star}
$$

h) Esperança matemätica da $\operatorname{SQT}^{*}\left(a_{j}.\right)$

$E\left[S Q T^{*}(a j).\right]=\tau^{\prime}\left(C-Q_{x} E_{1}^{-1} Q_{x}^{\prime}\right) \tau+(v-1) \sigma^{2}, j u s$ tificando-se, assim, a associação de $(v-1)$ graus de liberdade à soma de quadrados de tratamentos, ajustada para blocos e regressão

0 autor demonstra que a soma de quadrados do resỉduo, dividida por $\sigma^{2}$, ajustada para a regressão, tem distribuição $x^{2}$ central e as demais, $x^{2}$ não central, jä que o modelo é suposto de efeitos fixos. Ademais, afirma que é correta a ut lização do teste $F$ para o teste das hipóteses de que os efeitos de tratamentos e os coeficientes de regressão são todos nulos.

Finalmente, determina a matriz de dispersão para as estimativas dos efeitos de tratamentos ajustados para blo cos e regressão. Determina, ainda, uma fórmula para o cālculo da variância média do contraste entre duas médias de tratamentos ajustadas para blocos e regressão. 
3. DESENVOLVIMENTO TEORICO

\subsection{Ensaios em Blocos Completos Aumentados (Blocos de Federer!}

3.1.1. Definição dos parâmetros

0: parãmetros que caracterizam os delineamentos em blocos completos aumentados serão representados, neste trabalho, por:

c è o nūmero de tratamentos comuns;

b è o nümero de blocos;

$z$ é o nümero de tratamentos regulares;

$r_{j}$ é o nümero de rejetição do tratamento $j(j=1,2, \ldots, c, c+1, \ldots, c+z)$

$k_{i} \bar{e}$ o número de parcelas no broco $i(i=1,2, \ldots, b)$

$N$ é o número total de parcelas $(N=b c+z)$

3.1.2. Um modelo linear

0 modelo linear utilizado foi $y_{i j}=\mu+\beta_{i}+t_{j}+e_{i j}$, 
que tambēm pode ser representado matricialmente por $\underset{\sim}{Y}=X_{\sim}+\underset{\sim}{\varepsilon}$, onde Y é o vetor dos valores observados, de dim $(b c+z) \times 1$; $Y^{\prime}=\left[y_{11} y_{12} \cdots y_{1} c^{y_{1 c+1}} y_{1 c+2} \cdots y_{1 c+z_{1}} y_{21} y_{22} \cdots y_{2 c} y_{2 c+1} y_{2 c+2} \cdots\right.$ $\left.y_{2 c+z_{2}} \cdots y_{b 1} y_{b 2} \cdots y_{b c} y_{b c+1} y_{b c+2} \cdots y_{b c+z_{b}}\right]$

$X$ é a matriz do delineamento, de $\operatorname{dim}(b c+z) \times(b+c+z)$ e rank $(b+c+z-1)$

Efetuando-se convenientemente a partição da matriz $x$ obtēm-se

$$
x=\left[\begin{array}{l:l}
x_{1} & x_{2}
\end{array}\right]
$$

onde

$x_{1}$ é a matriz dos coeficientes dos efeitos de blocos, de dim $(b c+z) \times b ;$

$x_{2}$ e a matriz dos coeficientes dos efeitos de tratamentos, de $\operatorname{dim}(b c+z) \times(c+z)$;

巳 è o vetor dos parāmetros, de $\operatorname{dim}(b+c+z) \times 1$;

A partição do vetor $\theta$, correspondente à partição da

matriz $x \bar{e}$

onde

$$
\underline{\sim}^{\prime}=\left[\begin{array}{l:ll}
\beta^{\prime} & \underline{T}^{\prime} \\
\underline{\sim}^{\prime}
\end{array}\right]
$$

$\underset{\text { B }}{\mathrm{e}}$ o vetor dos efeitos de blocos que incluem a média geral, de $\operatorname{dim}(b \times 1)$;

I $\bar{e}$ o vetor dos efeitos de tratamentos, de $\operatorname{dim}(c+z) \times 1 ;$ 
$\underset{\sim}{\varepsilon}$ o vetor dos erros experimentais, sendo que

$$
\begin{aligned}
& \varepsilon \sim N\left(\emptyset, I \sigma^{2}\right) \text { e de } \\
& \operatorname{dim}(b c+z) \times 1
\end{aligned}
$$

3.1.3. Sistema de equações normais

0 sistema de equações normais, obtido atravēs do método dos quadrados minimos, é:

$$
X^{\prime} X{\underset{\sim}{\theta}}^{0}=X^{\prime} \underset{\sim}{Y}
$$

ou seja:

$$
\left[\begin{array}{cc}
x_{1}^{1} x_{1} & x_{1}^{\prime} x_{2} \\
x_{2}^{\prime} x_{1} & x_{2}^{\prime} x_{2}
\end{array}\right]\left[\begin{array}{c}
\underline{s}^{0} \\
\tau_{0}^{0}
\end{array}\right]=\left[\begin{array}{c}
x_{1}^{\prime} \\
x_{2}^{\prime}
\end{array}\right](3.1 .3 . b)
$$

As matrizes do sistema anterior são designadas

por:

$X_{j} X_{1}=K=\operatorname{Diag}\left\{K_{i}\right\}$ (matriz associada ao nümero de unidades expe rimentais por bloco);

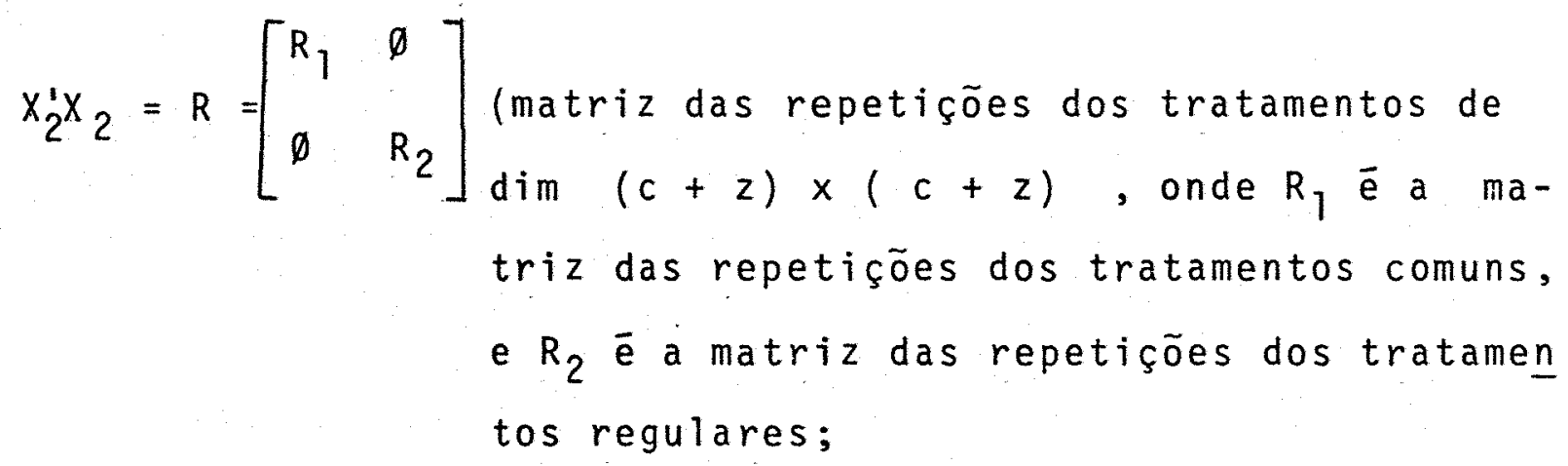

$x_{i} x_{2}=N^{\prime}=\left(\delta_{i j}\right) \quad$ (natriz de incidencia dos tratamentos em re 


$$
\begin{aligned}
& \text { lação aos blocos de dim bx }(c+z), \\
& \text { isto é: } \\
& \delta_{i j}=\left\{\begin{array}{l}
1, \text { se o tratamento j está no bloco } i ; \\
0, \text { caso contrārio. }
\end{array}\right.
\end{aligned}
$$

Assim, o sistema de equações em (3.1.3.b) pode ser escrito na forma

$$
\left[\begin{array}{ll}
K & N^{\prime} \\
N & R
\end{array}\right]\left[\begin{array}{c}
\underline{B}^{0} \\
\mathcal{\sim}_{0}^{0}
\end{array}\right]=\left[\begin{array}{cc}
X_{1}^{\prime} & \mathcal{\sim} \\
X_{2}^{\prime} & \mathcal{\sim}
\end{array}\right]=\left[\begin{array}{c}
\underset{\sim}{B} \\
\underset{I}{T}
\end{array}\right]
$$

onde,

$$
\begin{aligned}
& \stackrel{\beta}{\beta}^{0}=\left[\begin{array}{c}
\beta_{1}^{0} \\
\beta_{2}^{0} \\
\cdots \\
\beta_{b}^{0}
\end{array}\right] \text { para o vlocos, de dim }(b \times 1) ; \\
& \sim_{0}^{0}=\left[\begin{array}{c}
T_{01}^{0} \\
T_{02}^{0}
\end{array}\right] \bar{e} \text { o vetor de soluções de mïnimos quadrados } \\
& \tau_{01}^{0}=\left[\begin{array}{c}
t_{01}^{0} \\
t_{02}^{0} \\
\cdots \\
t_{0 c}^{0}
\end{array}\right] \text { para os tratamentos comuns, de dim }(c \times 1) \text {; }
\end{aligned}
$$




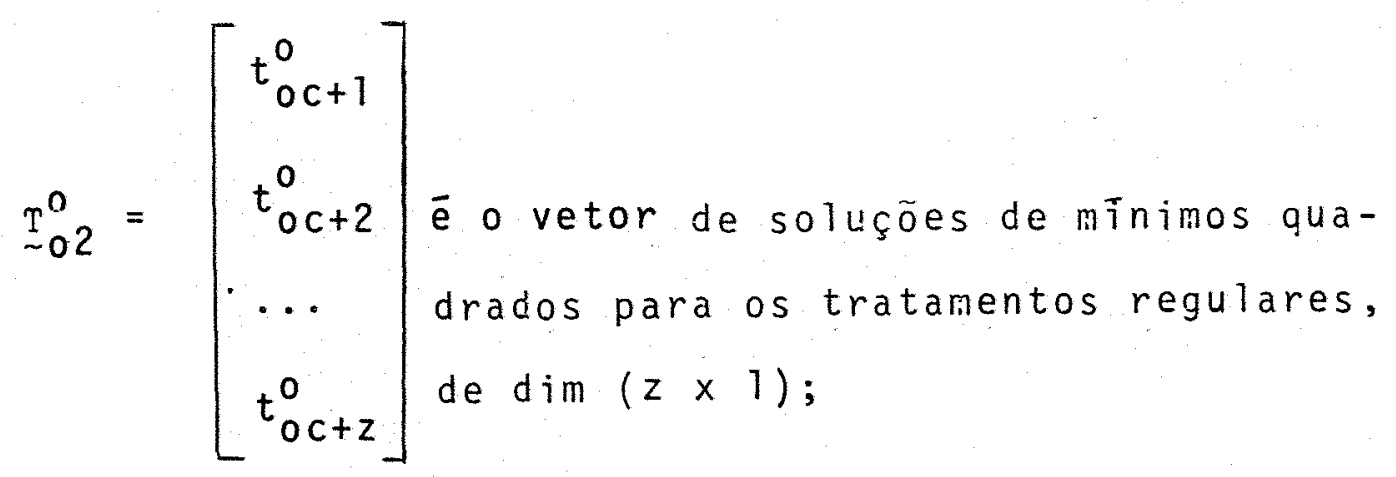

$B=\left[\begin{array}{c}c+z \\ \Sigma y_{1 j} \\ j=1 \\ c+z \\ \Sigma y_{2 j} \\ j=1 \\ \cdots \\ c+z \\ \Sigma y_{b j} \\ j=1\end{array}\right] \bar{e}$ o vetor dos totais observados para

$I=\left[\begin{array}{l}I_{1} \\ I_{2}\end{array}\right] \begin{aligned} & \bar{e} \text { o vetor dos totais observados dos tra } \\ & \text { tamentos, de dim }(c+z) \times 1\end{aligned}$

$\underline{T}_{1}=\left[\begin{array}{c}b \\ \Sigma_{y j} \\ i=1 \\ b \\ \Sigma y_{i 2} \\ i=1 \\ \cdots \\ b \\ \Sigma y_{i c} \\ i=1\end{array}\right]$ tamentos comuns, de dim (c x 1$)$ 


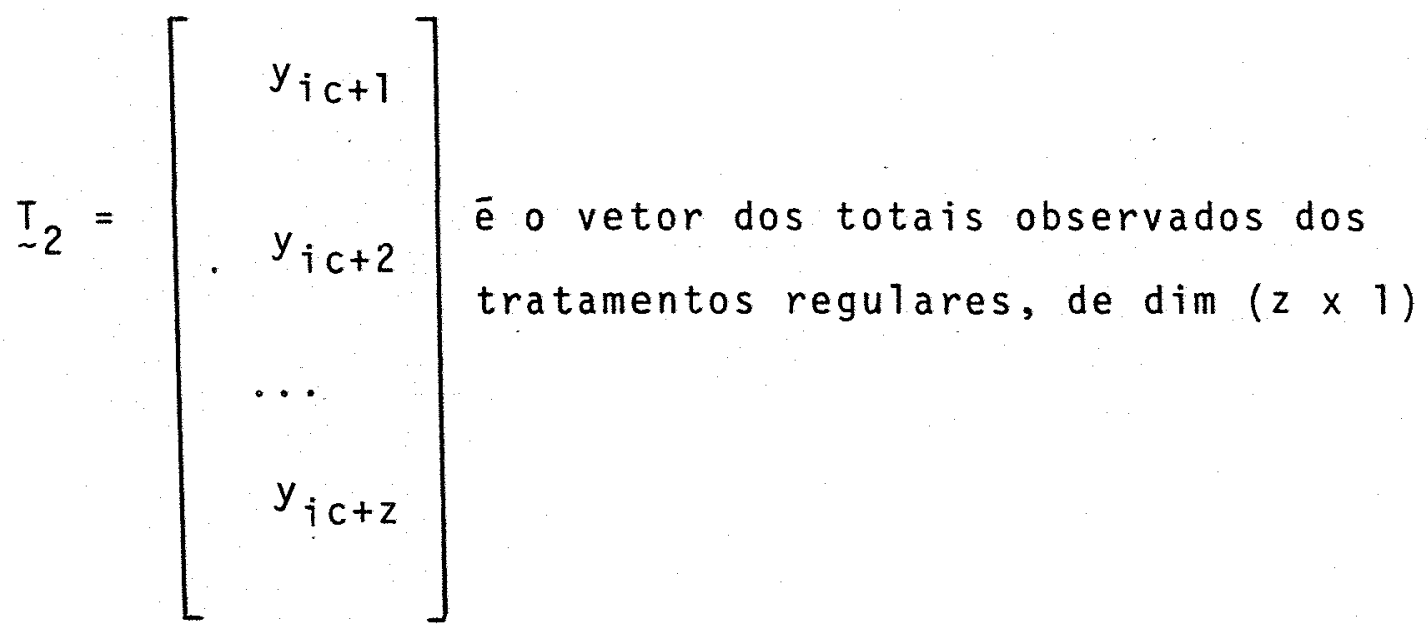

3.1.4. Estimação dos efeitos de tratamentos ajustados para blocos.

0 sistema (3.1.3.c) resulta nas seguintes equações :

$$
\begin{aligned}
& K \underline{\beta}^{0}+N^{1}{\underset{\sim}{T}}_{0}^{0}=\underset{\sim}{B} \quad(3.1 .4 . a) \\
& N \underline{\sim}^{0}+R{\underset{\sim}{T}}_{0}^{0}=\underset{\sim}{T} \quad(3.1 .4 . b)
\end{aligned}
$$

Para estimarem-se os efeitos de tratamentos, ajuㅗㅡ tados para blocos, os efeitos de blocos devem ser eliminados da equação (3.1.4.b). Com esse objetivo adiciona-se à equação $(3.1 .4 . b)$ a equação $(3.1 .4 . a)$, pré-multiplicada por $\left(-N K^{-1}\right)$. Isso resulta em:

$$
\left(R-N K^{-1} N^{\prime}\right){\underset{\sim}{T}}_{0}^{0}=I-N K^{-1} \underset{\sim}{B} \quad(3.1 .4 . c)
$$

que equivale a pré-multiplicar o sistema (3.1.3.c)pela matriz W, onde 
$W=\left[\begin{array}{ll}-N K^{-1} & I_{C+Z}\end{array}\right] \quad(3.1 .4 . d)$

\subsubsection{Método clāssico}

0 sistema de equações (3.1.4.c) pode ser escri to na forma

$$
\underline{C r}_{-0}^{0}=\underline{Q}
$$

onde $C$ e uma matriz simétrica, de característica $(c+z)-1$ e dimensões $(c+z) \times(c+z),{\underset{\sim}{0}}_{0}^{0} \bar{e}$ um vetor de dim $(c+z) \times 1$ e $Q$ é um vetor de $\operatorname{dim}(c+z) \times 1$, denominado de vetor de totais ajustados dos tratamentos. A matriz $c \bar{e}$

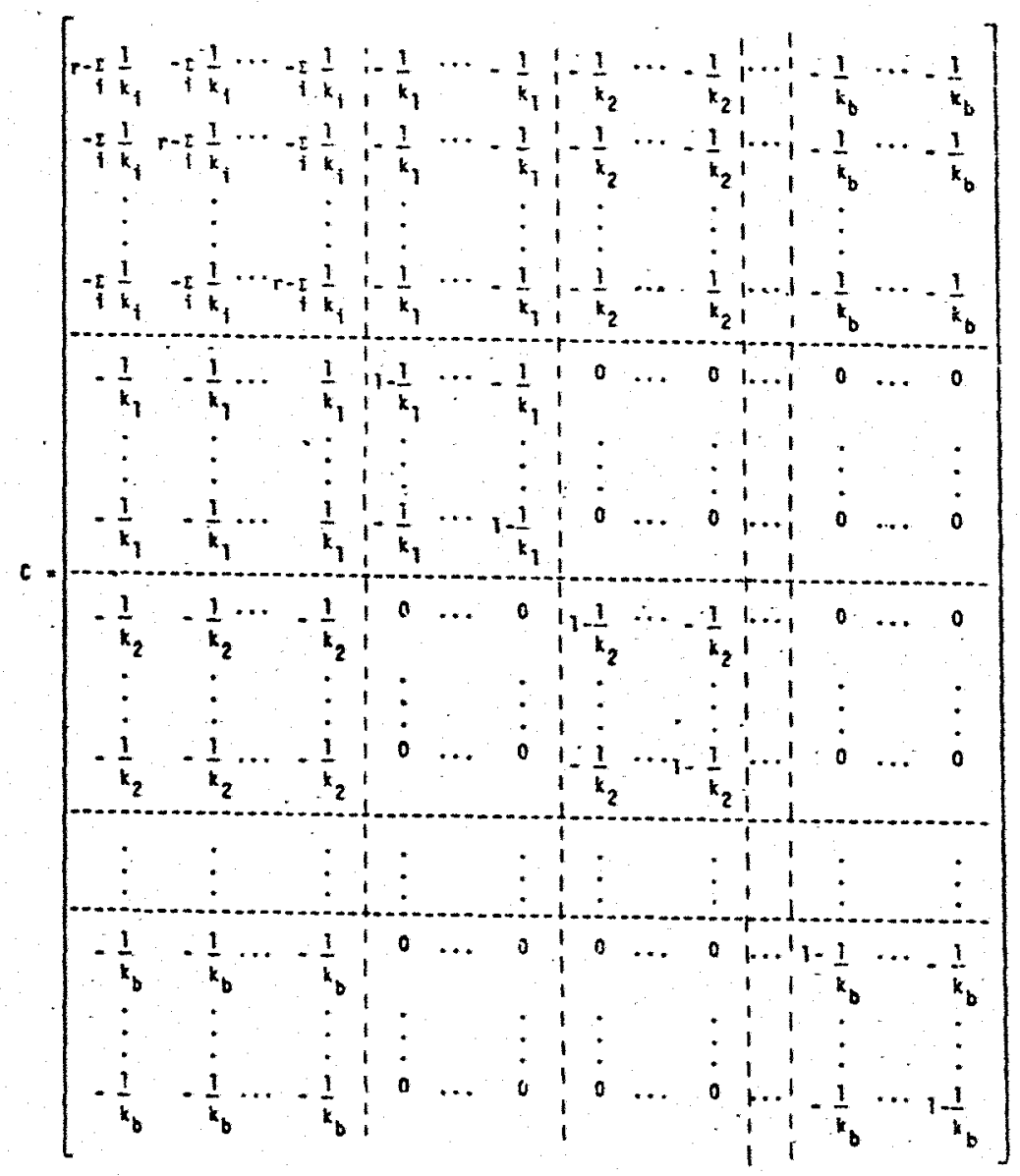


Como C é uma matriz singular, o sistema (3.1.4.c) pode ser resolvido, conforme PAVATE (1961) e PIMENTEL GOMES (1967) dentre outros, mediante a introdução de uma matriz A, com as mesmas dimensões de $C$, denominada "matriz de restrição", tal que a solução do sistema seja única. As restrições introduzidas resultam no sistema de equações, que pode ser escrito na forma :
$A \underset{\sim 0}{T}=\emptyset$

A matriz de restrição $A$, usando a restrição

$$
\sum_{i} \sum_{s} \frac{1}{k_{i}} t_{s}=0 \quad \text { onde }
$$

$t_{s}(s=1,2, \ldots c)$ são os efeitos dos tratamentos comuns

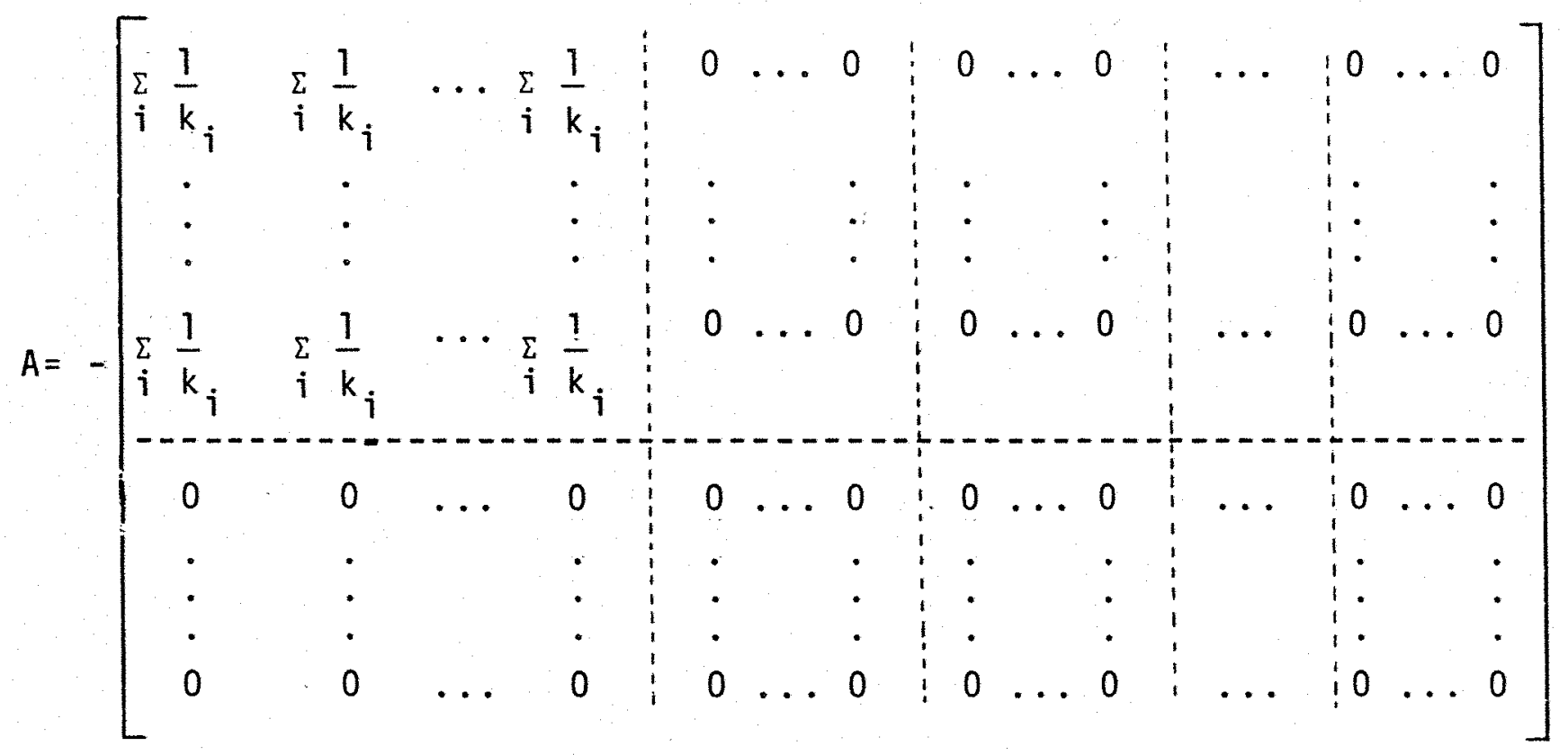




$$
\begin{array}{ll}
\text { De }(3.1 .4 .1 . a) \text { e } & (3.1 .4 .1 . b) \text { obtēm-se: } \\
(C+A){\underset{\sim}{T}}_{0}^{0}=\underset{\sim}{Q} & (3.1 .4 .1 . c)
\end{array}
$$

onde $C+A=M \bar{e}$ uma matriz não singular. Logo, a solução do sistema $\bar{e}$ dada por:

$$
\stackrel{T}{0}_{0}^{0}=M^{-1} \underline{Q}
$$

onde, a matriz M de $\operatorname{dim}(c+z) \times(c+z) \bar{e}$ dada por

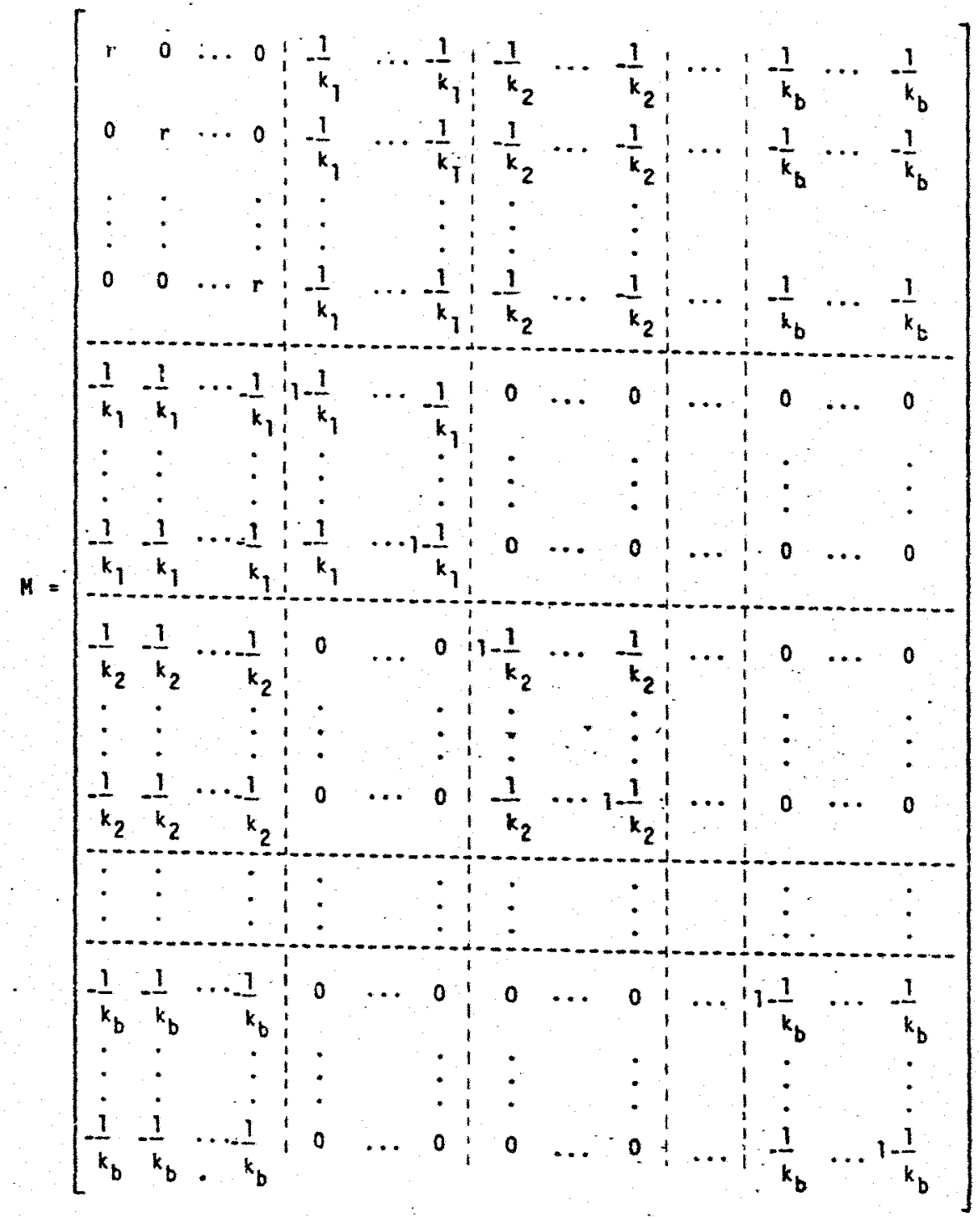


e a matriz $M^{-1}$ tem a forma:

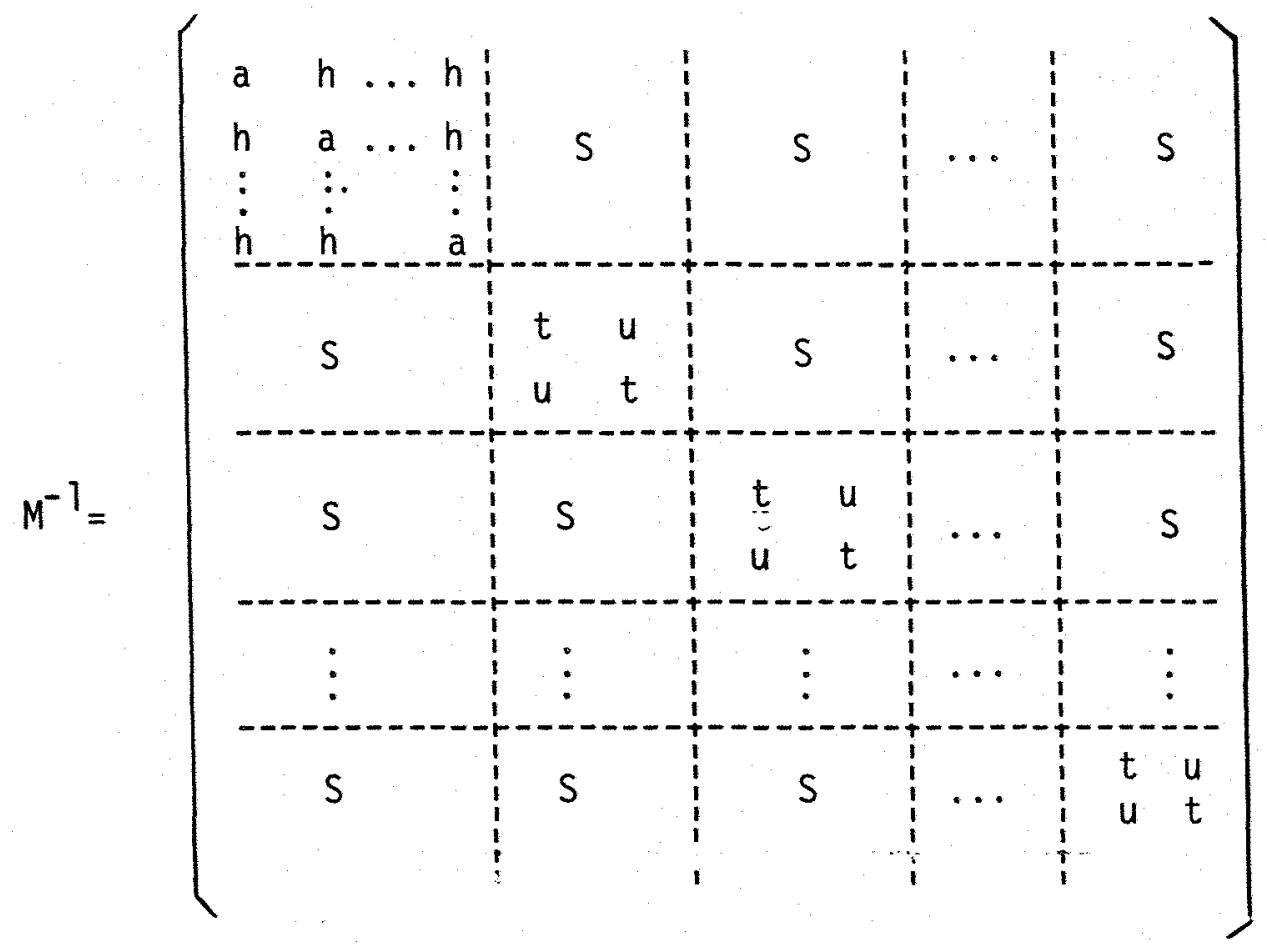

cujos elementos da matriz $\mathrm{M}^{-1}$ são obtidos pelas fórmulas:

$$
\begin{aligned}
& A=\frac{1}{c\left[b-\sum_{i=1}^{b} \frac{n_{i}}{k_{i}}\right]} \\
& a=s+\frac{1}{b}-\frac{1}{c \cdot b} \\
& h=s-\frac{1}{c \cdot b} \\
& t=s+\frac{1}{c}+1 \\
& u=s+\frac{1}{c}
\end{aligned}
$$

onde $S \vec{e}$ a matriz de constantes $S$;

$n_{j} \bar{e}$ o número de tratamentos regulares no bloco $i$;

$k_{i} \bar{e}$ o número de tratamentos comuns e regulares no bloco $i$ $\left(k_{j}=c+n_{j}\right)$;

c é o número de tratamentos comuns. 
3.1.5. Somas de quadrados

$$
\begin{aligned}
\text { Do modelo } & \underset{\sim}{Y}=X \underline{\sim}+\underset{\sim}{\varepsilon} \text {, obtēm-se } \\
& \underline{\sim}^{\prime} \underline{\sim}=\underline{Y}^{\prime} \underline{\sim}-\underline{\sim}^{\prime} X^{\prime} \underline{Y}
\end{aligned}
$$

e tomando-se a estimativa de $\theta$, resulta a soma de quadrados do residuo, ou seja:

$$
S Q R=\underline{Y}^{\prime} \underline{Y}-\underline{\theta}^{0} \cdot X^{\prime} \underline{Y},
$$

onde, $\underline{\theta}^{0} \times X^{\prime} \underline{y}$, definida como a soma de quadrados de parámetros, $\bar{e}$ :

$$
\begin{aligned}
& \text { SQPar }=\left[\begin{array}{cc}
B^{O^{\prime}} & \mathcal{T}^{0^{\prime}} \\
\mathcal{O}^{0}
\end{array}\right]\left[\begin{array}{c}
B \\
\sim \\
I
\end{array}\right] \\
& =\underline{\beta}^{0^{\prime}} \underset{\sim}{B}+{\underset{T}{T^{0}}}^{0^{\prime}} \underset{\sim}{T}
\end{aligned}
$$

De $3.1 .4 . a$ tem-se que $\underline{B}^{0}=K^{-1}\left(\underset{B}{B}-N^{\prime}{\underset{\sim}{T}}_{0}^{0}\right)$,

$\log 0$

$$
\begin{aligned}
& \text { SQPar }=\left(\underset{\sim}{B}-N^{\prime}{\underset{\sim}{T}}_{0}^{0}\right)^{\prime} K^{-1} \underline{B}+{\underset{\sim}{T}}_{0}^{0^{\prime}} I
\end{aligned}
$$

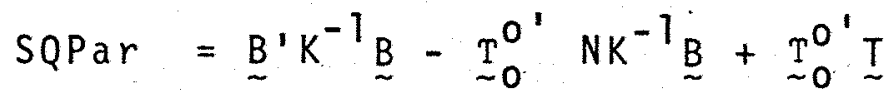

$$
\begin{aligned}
& \text { Sopar }={\underset{\sim}{I_{0}}}_{0}^{\prime}\left(\underset{\sim}{I}-N K^{-1} \underline{B}\right)+\underline{B}^{\prime} K^{-1} \underline{B} \\
& \text { SoPar }=\underset{\sim}{T_{0}^{0}} \underline{Q}+\underset{\sim}{B^{\prime}} K^{-1} \underset{\sim}{B}
\end{aligned}
$$

Substituindo-se $(3.1 .5 . b)$ em $(3.1 .5 . a)$, tem-se 


$$
S Q R=\underset{\sim}{Y^{\prime}} \underline{Y}-\underline{\sim}^{O^{\prime}} \underline{Q}-\underset{\sim}{B^{\prime} K^{-1}} \underset{\sim}{B} \quad 3.1 .5 . C
$$

onde

Y' ${ }^{\prime}$ é a soma de quadrados total (SQT)

$\mathrm{T}_{0} \underline{Q}$ é a soma de quadrados de tratamentos ajustada parablocos (SQT aj. $\left._{\text {. }}\right)$

$\underline{B}^{\prime} k^{-1} \underline{B} \bar{e}$ a soma de quadrados de blocos e média.

3.1.6. Esperança matemātica das somas de quadrados

3.1.6.1. Esperança matemática de $\underline{Y}^{\prime} \underline{Y}$

A soma de quadrados devida a total é:

$$
\begin{aligned}
& S Q T=\underline{Y^{\prime}} \underline{Y} \\
& =\left(\theta^{\prime} X^{\prime}+\underline{\varepsilon}^{\prime}\right)(\underset{\sim}{X}+\underline{\sim}) \\
& =\underline{\theta}^{\prime} X^{\prime} X \underline{\sim}+\theta^{\prime} X^{\prime} \underline{\varepsilon}+\varepsilon^{\prime} X \underline{\sim}+\underline{\sim}^{\prime} \underline{\sim}^{\prime}
\end{aligned}
$$

têm-se, então, que:

$$
\begin{aligned}
& E(S Q T)={\underset{\sim}{\theta}}^{\prime} X^{\prime} X \underset{\sim}{\theta}+E\left({\underset{\sim}{\varepsilon}}^{\prime} \underline{\sim}^{\prime}\right) \quad(3.1 .6 .1 . a) \\
& X \cdot X=\left[\begin{array}{ll}
K & N \\
N & R
\end{array}\right] \\
& \underline{\sim}^{\prime} X ' X \underset{\sim}{\theta}=\left[\begin{array}{ll}
\underline{\beta}^{\prime} & \underline{T}^{\prime}
\end{array}\right]\left[\begin{array}{ll}
K & N^{\prime} \\
N & R
\end{array}\right]\left[\begin{array}{c}
\beta \\
\underset{\tilde{I}}{\beta}
\end{array}\right] \\
& =\underline{\sim}^{\prime} k \underline{\underline{\beta}}+\underline{\sim}^{\prime} N \underline{\beta}+\underline{\sim}^{\prime} N^{\prime} \underline{\sim}+\underset{\sim}{T^{\prime}} \underline{R}_{\underline{T}} \\
& =\underset{\sim}{T}{ }^{\prime} \underset{\sim}{T}+2 \underset{\sim}{\beta} N^{\prime} \underset{\sim}{T}+\underset{\sim}{\beta}{ }^{\prime} \underset{\sim}{\beta}
\end{aligned}
$$

Substituindo-se $(3.1 .6 .1 . b)$ em $(3.1 .6 .1 . a)$, tem-se:

$$
\begin{aligned}
& E(S Q T)={\underset{\sim}{T}}^{\prime} \mathbb{R}_{\sim}+2 \underline{\sim}^{\prime} N^{\prime} \underline{T}+\underset{\sim}{\beta^{\prime}} \underline{\sim}_{\sim}^{\beta}+T R\left[E\left(\underset{\sim}{\varepsilon \varepsilon^{\prime}}\right)\right] \\
& ={\underset{\sim}{T}}^{\prime} R \underset{\sim}{P}+2 \underline{\sim}^{\prime} N^{\prime} \underset{\sim}{T}+{\underset{\sim}{\beta}}^{\prime} k \underset{\sim}{\beta}+(b \cdot c+z) \sigma^{2}(3.1 .6 .1 . c)
\end{aligned}
$$


3.1.6.2. Esperança matemātica de $\underset{\sim}{B^{\prime}} k^{-1} \underset{\sim}{B}$

A soma de quadrados de blocos è dada por:

$\dot{S} Q B=\underline{B}^{\prime} K^{-1} \underline{B}=\underline{Y} X^{\prime} X_{1} k^{-1} X_{1}^{\prime} \underline{Y}$

Tem-se, então, que:

$$
\begin{aligned}
& E(S Q B)=E\left(\underset{\sim}{Y^{\prime}} X_{1} k^{-1} X_{\underset{1}{\prime} \underset{\sim}{Y}}\right) \\
& =E(\underset{\sim}{\theta^{\prime}} X^{\prime}+\underbrace{\prime}_{\sim}) X_{1} K^{-1} X^{\prime}(X \theta+\underset{\sim}{\varepsilon}) \\
& =\theta_{\sim}^{\prime} X^{\prime} X_{1} K^{-1} X_{1}^{\prime} X \theta+E\left(\underset{\sim}{\varepsilon^{\prime}} X_{1} K^{-1} X_{1}^{\prime} \underset{\sim}{\varepsilon}\right)(3.1 .6 .2 a) \\
& x^{\prime} x_{1}=\left[\begin{array}{c}
x_{1}^{\prime} \\
-x_{2}^{\prime}
\end{array}\right] x_{1}=\left[\begin{array}{l}
K \\
N
\end{array}\right] \\
& x_{1}^{\prime} x=x_{1}^{\prime}\left[\begin{array}{l:l}
x_{1} & x_{2}
\end{array}\right]=\left[\begin{array}{l:l}
K & N^{\prime}
\end{array}\right] \\
& x^{\prime} x_{1} K^{-1} x_{1} x=\left[\begin{array}{l}
K \\
N
\end{array}\right] K^{-1}\left[\begin{array}{l:l}
K & N^{\prime}
\end{array}\right]=\left[\begin{array}{lll}
K & N^{\prime} \\
N & N K^{-1} & N^{\prime}
\end{array}\right] \\
& \underset{\sim}{\theta} X^{\prime} x_{1} K^{-1} X_{1}^{\prime} X_{\theta}=\left[\begin{array}{ll}
\beta^{\prime} & N^{\prime} \\
\sim & \sim
\end{array}\right]\left[\begin{array}{cc}
K & N^{\prime} \\
N & N K^{-1} N
\end{array}\right]\left[\begin{array}{l}
\underset{\sim}{R} \\
\underset{R}{B}
\end{array}\right]=
\end{aligned}
$$$$
={\underset{\sim}{\beta}}^{\prime} K \underline{\sim}+\underline{\sim}^{\prime} N \underline{\sim}+\underline{\sim}^{\prime} N^{\prime} \underset{\sim}{T}+\underset{\sim}{T^{\prime}} N^{-1} N^{\prime} \underline{T}
$$$$
=\underline{\beta}^{\prime} \underline{K}_{\underline{\beta}}+2 \underline{\beta}^{\prime} N \underset{\mathcal{T}}{N}+\underline{T}^{\prime} N K^{-1} N^{\prime} \underset{\sim}{T}
$$

Substituindo-se $(3.1 .6 .2 . b)$ em $(3.1 .6 .2 . a)$, segue-se que:

$$
\begin{aligned}
& E(S Q B)=\underset{\sim}{\beta^{\prime}} \underset{\sim}{\beta}+\underset{\sim}{2 \beta} ! N T+{\underset{\sim}{T}}^{\prime} N K^{-1} N^{\prime} \underset{\sim}{T}+ \\
& \operatorname{TR}\left[X_{1} K^{-1} X_{1}^{\prime} E(\underset{\sim}{\varepsilon} \underbrace{\prime})\right]
\end{aligned}
$$$$
\text { Mas, } E\left(\underline{\varepsilon}^{\prime}\right)=\sigma^{2} I, \in \operatorname{TR}\left(X_{1} K^{-1} X_{1}^{\prime}\right)=\operatorname{TR}\left(I_{b}\right)=b
$$ 
$\log 0$,

$$
\begin{aligned}
& E(S Q B)=\underline{\sim}^{\prime} N K^{-1} N^{\prime} \underset{\sim}{T}+{\underset{\sim}{B}}^{\prime} K \underset{\sim}{\beta}+2 \underline{\sim}^{\prime} N^{\prime} \underset{\sim}{T}+b \sigma^{2} \text { (3.1.6.1.c) } \\
& \text { 3.1.6.3. Esperança matemātica de }{\underset{\sim}{0}}_{0}^{0^{\prime}} Q \\
& \text { Sabe-se que: } \\
& T_{0}^{0^{\prime}} \underline{Q}=\underline{Q}^{\prime} M^{-1} \underline{Q}=Q^{\prime} M^{-1} \underline{Q}
\end{aligned}
$$$$
\text { Pode-se comprovar a seguinte relação } \underline{Q}=W X^{\prime} \underline{\sim} \text { e, }
$$
então, considerando-se o modelo como em 3.1 .2 , segue-se que:

$$
\begin{aligned}
& E\left(\underset{\sim}{\stackrel{\varphi}{O} 0^{\prime}} \underset{\sim}{Q}\right)=E\left(\underline{\sim}^{\prime} X W^{\prime} M^{-1} W X^{\prime} \underset{\sim}{Y}\right) \\
& =E\left(\underset{\sim}{\theta^{\prime}} X^{\prime}+\underset{\sim}{\varepsilon^{\prime}}\right) X W^{\prime} M^{-1} W X^{\prime}(\underset{\sim}{X}+\underset{\sim}{\theta}) \\
& E\left(T_{\sim}^{0} \underset{\sim}{Q}\right)=\underline{\sim}^{\prime} X^{\prime} X W^{\prime} M^{-1} W X^{\prime} X \underset{\sim}{\theta}+E\left(\underline{\sim}^{\prime} X W^{\prime} M^{-1} W X^{\prime} \varepsilon\right)
\end{aligned}
$$

Como

$$
\begin{aligned}
W X^{\prime} X=\left[\begin{array}{ll}
-N K^{-1} & I(c+z)
\end{array}\right]\left[\begin{array}{ll}
K & N^{\prime} \\
N & R
\end{array}\right] & =\left[\begin{array}{lll}
\emptyset & R & -N K^{-1} N^{\prime}
\end{array}\right] \\
& =\left[\begin{array}{ll}
\emptyset & 6
\end{array}\right]
\end{aligned}
$$

então

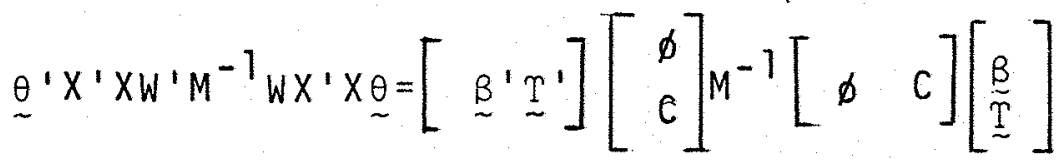

$$
\begin{aligned}
& =\underset{\sim}{\prime} \mathrm{CM}^{-1} \mathrm{C} \underset{\sim}{\mathrm{T}}
\end{aligned}
$$

OGAWA (1974) e IEMMA (1985) mostram que a ma triz $\mathrm{M}^{-1}$ é uma inversa condicional de $\mathrm{C}$, logo $\mathrm{CM}^{-1} \mathrm{C}=\mathrm{C}$. 


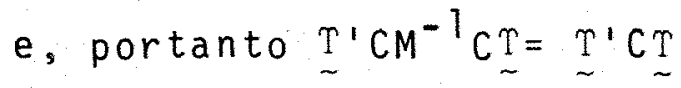

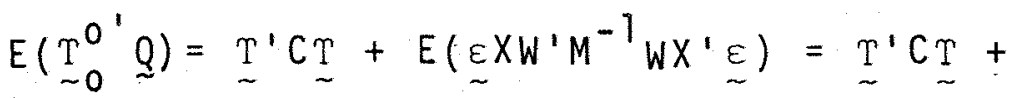

$$
\begin{aligned}
& +E\left[T R\left(X W^{\prime} M^{-1} W X^{\prime}{\underset{\sim}{\prime}}_{\sim}^{\prime} \varepsilon\right)\right] \\
& E\left(T_{0}^{0^{\prime}} Q\right)={\underset{\sim}{T}}^{\prime}{\underset{\sim}{T}}_{\sim}+T R\left[X W^{\prime} M^{-1} W X^{\prime} E\left({\underset{\sim}{\varepsilon}}^{\prime} \underset{\sim}{\varepsilon}\right)\right] \\
& ={\underset{\sim}{T}}^{\prime} C_{\sim} \underset{\sim}{T}+\operatorname{TR}\left(M^{-1} W X^{\prime} X W^{\prime}\right) \sigma^{2} \\
& =\underset{\sim}{T}{ }^{\prime} C \underset{\sim}{T}+\operatorname{TR}\left(M^{-1} C\right) \sigma^{2}
\end{aligned}
$$

Como $M^{-1} C$ é uma matriz idémpotente

então

$\operatorname{TR}\left(M^{-1} C\right)=\operatorname{rank}\left(M^{-1} C\right)=\operatorname{rank}(C)=C+z-1$, pois $M \bar{e}$ não singu lar.

Logo, $E\left(\underline{T}_{0}^{0} q\right)=E\left(S Q T_{a j}\right)=\underline{\sim}^{\prime}{ }^{\prime} \underset{\sim}{T}+(c+z-1) \sigma^{2}$

$$
(3.1 .6 .3 . b)
$$

3.1.6.4. Esperança matemätica da SQR

De 3.1.5.c, tem-se:

$$
E(S Q R)=E\left(\underline{\sim}^{\prime} \underline{Y}\right)-E\left({\underset{\sim}{O}}_{0}^{O^{\prime}} \underset{\sim}{Q}\right)-E\left({\underset{\sim}{B K}}^{-1} \underset{\sim}{B}\right)
$$

Substituindo $(3.1 .6 .1 . c), \quad(3.1 .6 .3 . b)$

$(3.1 .6 .2 . c)$ em $(3.1 .6 .4 . a)$, vem:

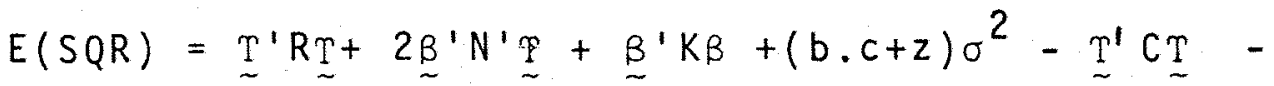

$$
\begin{aligned}
& (c+z-1) \sigma^{2}-\underline{\sim}^{\prime} N K^{-1} N^{\prime} \underset{\sim}{T}-\underset{\sim}{\beta^{\prime}} \underset{\sim}{\beta}-2 \underline{\sim}^{\prime} N^{\prime} \underset{\sim}{T}-b \sigma^{2} \\
& (3.1 .6 .4 . b)
\end{aligned}
$$


Simplificando-se $(3 \cdot 1 \cdot 6.4 . b)$, tem-se:

$$
E(S Q R)=(b-1)(c-1) \sigma^{2} \quad(3.1 .6 .4 . c)
$$

3.1.7. Quadro da anālise de variāncia

o quadro da anālise de variāncia, com as esperanças dos quadrados médios, pode ser apresentado na seguinte forma :

Esquema da anāi ise de variāncia

\begin{tabular}{|c|c|c|c|}
\hline Causas da Variação & G.L. & Q.M. & $E\left(Q . M_{.}\right)$ \\
\hline Blocos & $b-1$ & $S Q B /(b-1)$ & \\
\hline $\begin{array}{l}\text { Tratamentos (ajustados } \\
\text { para blocos) }\end{array}$ & $c+z-1$ & $\mathrm{SQT}_{\mathrm{aj} .} /(c+z-1)$ & $\sigma^{2}+\tau c T /(c+z-1)$ \\
\hline Residuo & $(b-1)(c-1)$ & $S Q R /(b-1)(c-1)$ & $\sigma^{2}$ \\
\hline
\end{tabular}


3.2. Anālise de Covariāncia em Blocos Completos Aumentados (Blocos de Federer)

3.2.1. Modelo matemático

Para o delineamento em questão supõe-se o mode10 linear de covariancia de efeito fixo:

$$
y_{i j}=\mu+\beta_{i}+t_{j}+\gamma_{x_{i j}}+e_{i j}, \quad, \quad(3.2 \cdot 1 \cdot a)
$$

com: $\quad i=1,2, \ldots, b$

$$
j=1,2, \ldots, c+z
$$

onde,

$y_{i j}$ é o valor observado da parcela do bloco $i$ que recebeu o tratamento $j$;

$\mu \quad \bar{e}$ a média geral;

${ }^{\beta_{i}} \bar{e}$ o efeito do bloco i;

$t_{j}$ é o efeito do tratamento j;

$\gamma \quad \bar{e}$ o coeficiente da regressão linear de $Y$ em relação a $X$;

$x_{i j}=x_{i j}-\bar{x}$, onde $x_{i j}$ são os valores observados da variāvel auxiliar (covariāvel);

$e_{i j}$ é o erro experimental associado à observação $y_{i j}$, ónde se supõe que os $e_{i j}$ 's são normalmente distribuídos, com média zero e variāncia ${ }^{2}$

$$
\text { o efeito } t_{j} \text { envolve } t_{s}(s=1,2, \ldots, c) \text { e }
$$
$t_{r_{s^{\prime}}}\left(s^{\prime}=c+1, c+2, \ldots, c+z\right)$, que são os efeitos dos tratamen tos comuns e regulares, respectivamente.

Usando-se a forma matricial, o modelo (3.2.1.a)

fica: 


$$
\underline{Y}=\underset{\sim}{X \theta}+\underset{\sim}{\varepsilon} \quad, \quad(3.2 .1 . \mathrm{b})
$$

onde,

$Y \bar{e}$ o vetor dos valores observados, de $\operatorname{dim}(b . c+z) \times 1$; $X$ é a matriz dos coeficientes dos parāmetros, de dim

$$
(b c+z) \times(c+z+b+1) \text { e } \operatorname{rank}(c+z+b)
$$

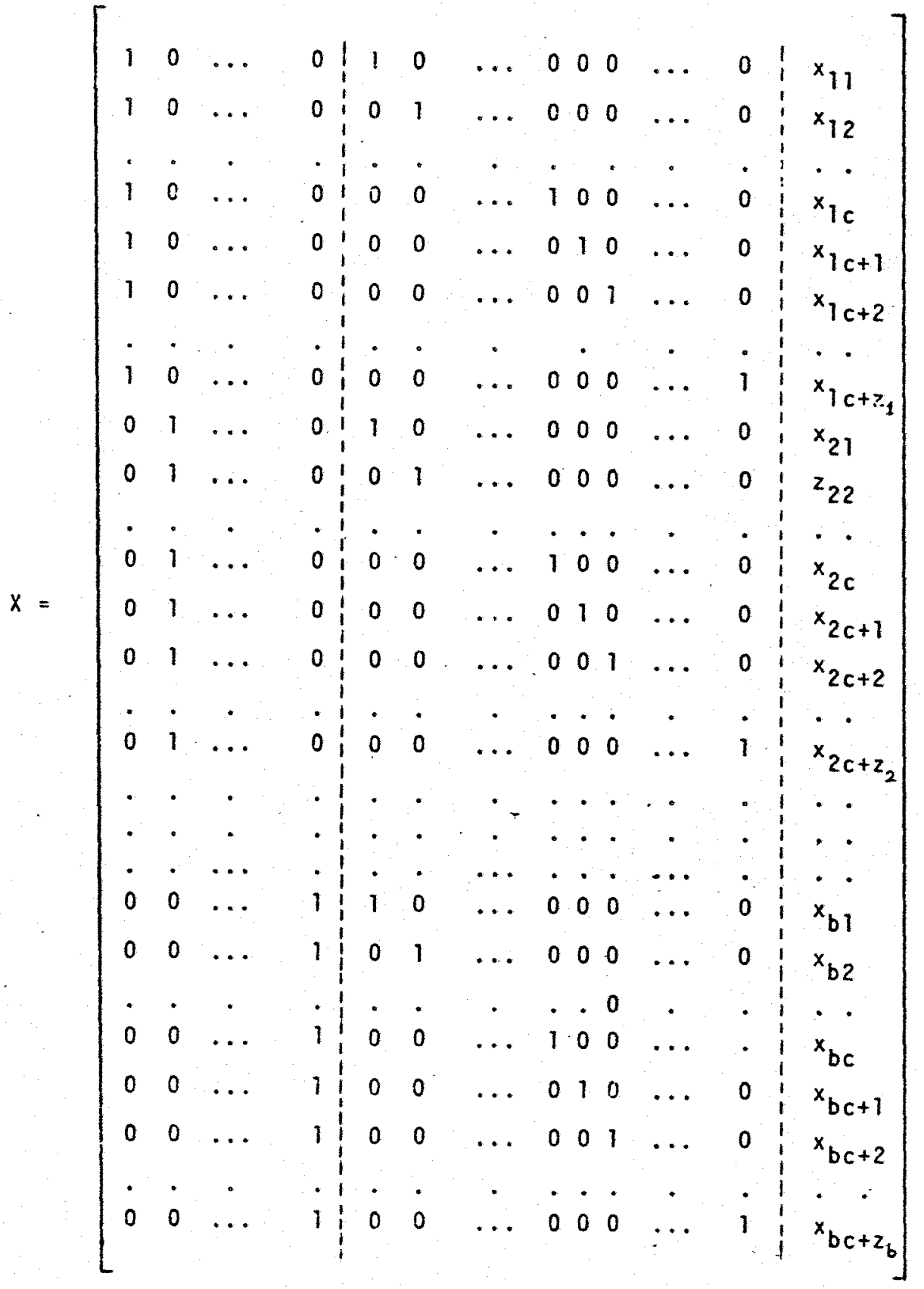


Observe que a matriz $x$, a menos da coluna da covariāvel é igual a matriz $X$ do item 3.1.2.

Éfetuando-se convenientemente a partição da matriz $x$ obtēm-se:

$$
x=\left[\begin{array}{l:l:l}
x_{1} & x_{2} & x_{3}
\end{array}\right]
$$

onde,

$x_{1}$ é a matriz dos coeficientes dos efeitos de blocos, de $\operatorname{dim}(b c+z) \times b ;$

$x_{2}$ é a matriz dos coeficientes dos efeitos de tratamentos, de $\operatorname{dim}(b c+z) \times(c+z)$;

$x_{3}$ é a matriz dos valores observados da variāvel auxiliar, de $\operatorname{dim}(b c+z) \times 1$

$\stackrel{\theta}{\text { è }}$ o vetor dos parāmetros, de $\operatorname{dim}(1+b+c+z) \times 1$.

A partição do vetor $\underset{\sim}{\theta}$, correspondente à partição da matriz $X \bar{e}$ :

$$
\underline{\sim}^{\prime}=\left[\begin{array}{l:l:l}
\beta^{\prime} & \Gamma^{\prime} & \gamma \\
\hdashline & \sim &
\end{array}\right]
$$

onde,

$\underset{\sim}{B}, \underset{\sim}{T}$ e $\underset{\sim}{\varepsilon}$ têm o mesmo significado jā descrito no item 3.1 .2 e com aquelas dimensões, respectivamente;

$\gamma$ é o coeficiente da regressão linear de $Y$ em relação a $X$. 


\subsubsection{Sistema de equações normais}

0 sistema de equações normais, obtido através do mêtodo dos quadrados minnimos, è dado por

$$
X^{\prime} X \underline{\underline{\theta}}^{0}=X^{\prime} Y \quad 3.2 .2 . a
$$

ou seja,

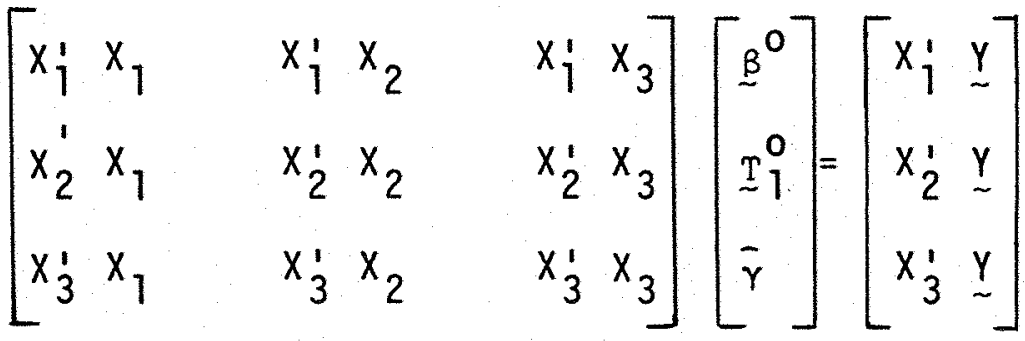

onde,

$x_{1}^{\prime} x_{1}, x_{2}^{\prime} x_{2}$ e $x_{1}^{\prime} x_{2}$ têm o mesmo significado jä descrito no item 3.1 .3 página 29 ;

$x_{j}^{\prime} X_{3}=B_{x}$ è a matriz associada ao número de covariāveis por bloco, de $\operatorname{dim}(b \times 1)$

$$
B_{x}=\left[\begin{array}{c}
c+z \\
\sum_{j=1} x_{1 j} \\
c+z \\
\sum_{j=1}^{c+z} x_{2 j} \\
\cdots \cdots \\
c+z \\
\sum_{j=1}^{c} x_{b j}
\end{array}\right]
$$

$x_{2}^{\prime} x_{3}=T_{x}$ e a matriz de totais de tratamentos (comuns e regu lares) para a covariável, de $\operatorname{dim}(c+z) \times 1$ 


$$
T_{x}=\left[\begin{array}{c}
b \\
\sum_{i=1} x_{i 1} \\
b \\
\sum_{i=1} x_{i 2} \\
\ldots \\
v_{i=1} x_{i c} \\
\\
x_{i c+1} \\
x_{i c+2} \\
\ldots \\
x_{i c+z}
\end{array}\right]
$$

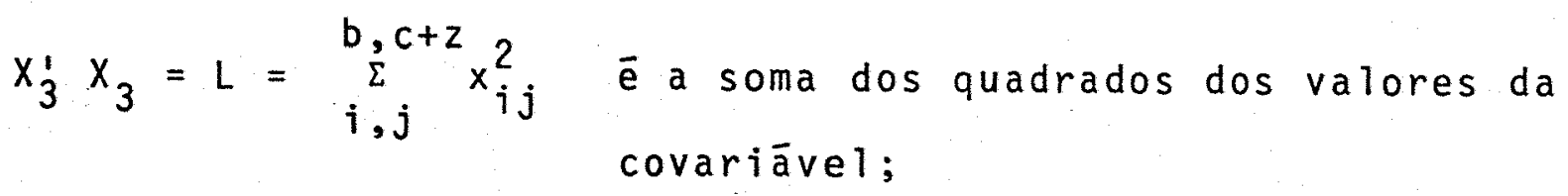

$$
\text { Assim, o sistema de equações em (3.2.2.b) pode }
$$

ser escrito na forma:

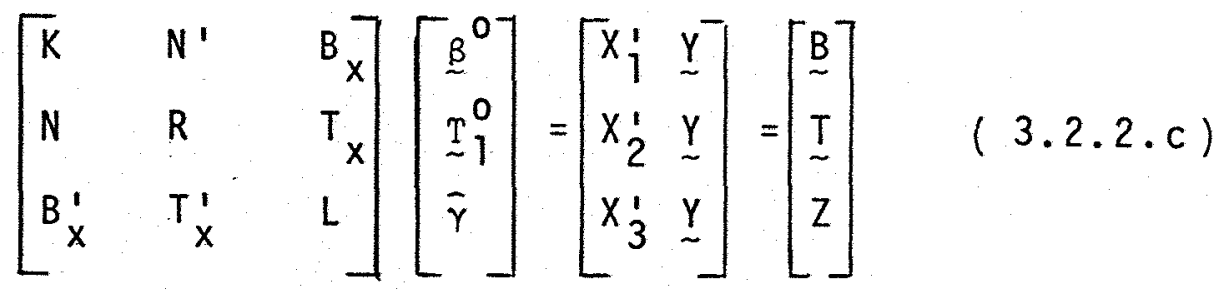

onde,

$$
\underline{\beta}^{0}=\left[\begin{array}{c}
\beta_{1}^{0} \\
\beta_{2}^{0} \\
\cdots \\
\beta_{b}^{0}
\end{array}\right] \text { é c vetor solução de minimos para blo }
$$


$I_{1}^{0}=\left[\begin{array}{c}0 \\ T_{11} \\ 0 \\ T_{12}\end{array}\right]$ é o vetor solução de mînimos quadrados para os
tratamentos, de dim $(c+z) \times 1 ;$
$\sim_{11}^{0}=\left[\begin{array}{c}t_{1}^{0} \\ t_{2}^{0} \\ \cdots \\ t_{c}^{0}\end{array}\right]$ é vetor solução de minimos quadrados para os
tratamentos comuns, de dim (c x 1$)$; $T_{12}^{0}=\left[\begin{array}{c}t_{c+1}^{0} \\ t_{c+2}^{0} \\ \cdots \\ t_{c+z}^{0}\end{array}\right]$

é o vetor solução de minnimos quadrados para os tratamentos regulares, de $\operatorname{dim}(z \times 1)$;

$\bar{y} \quad \bar{e}$ a estimativa do coeficiente da regressão $1 j-$ near de $Y$ em relação a $X$;

$z=\sum_{i}^{b} \sum_{j}^{c+z} x_{i j} y_{i j}$ é a soma dos produtos da variāvel de observação pela covariāvel;

B e I têm o mesmo significado descrito no item 3.1 .3 e com aquelas dimensões, respectivamente.

3.2.3. Estimação dos efeitos de tratamentos ajustados para blocos e regressão

0 sistema $3.2 .2 . c$ resulta nas seguintes 
equações:

$$
\begin{aligned}
& \mathrm{K} \underset{\sim}{\beta^{0}}+N^{1} \stackrel{T}{\mathcal{T}}_{j}^{0}+B_{X} \hat{\gamma}=\underset{\sim}{B} \\
& N \underline{\sim}^{0}+R \underline{\sim}_{1}^{0}+T_{x} \bar{\gamma}=I \\
& B_{X}^{1} \underline{B}^{0}+T_{X}^{1} \stackrel{T}{1}^{0}+L \bar{\gamma}=Z
\end{aligned}
$$

Para estimarem-se os efeitos de tratamentos, ajustados parablocos e regressão, os efeitos de blocos devem ser elimina dos do sistema 3.2.2.a. Com esse objetivo, prë-multiplicou -se 3.2.3.a por $\left(-N^{-1}\right)$ e por $\left(-B_{x}^{1} K^{-1}\right)$ e adiciona-se às equações $3.2 .3 . b$ e 3.2.3.c respectivamente. Isso resulta

em :

$$
\begin{aligned}
& \left(R-N K^{-1} N^{\prime}\right){\underset{\sim}{T}}_{1}^{0}+\left(T_{X}-N K^{-1} B_{X}\right) \bar{\gamma}=\underset{\sim}{T}-N K^{-1} \underset{\sim}{B} \\
& \left(T_{X}^{1}-B_{X}^{\prime} K^{-1} N^{\prime}\right) \underset{\sim}{\stackrel{T}{T}}+\left(L-B_{X}^{\prime} K^{-1} B_{X}\right) \bar{\gamma}=Z-B_{X}^{\prime} K^{-1} \underset{\sim}{B}
\end{aligned}
$$

Fazendo-se

$$
\begin{aligned}
& \left(R-N K^{-1} N^{\prime}\right)=C, \operatorname{de} \operatorname{dim}(c+z) \times(c+z) \\
& \left(T_{X}-N K^{-1} B_{X}\right)=Q_{X}, \operatorname{dedim}(c+z) \times 1 \\
& \left(\underset{\sim}{T}-N K^{-1} \underline{B}\right)=\underline{Q}, \operatorname{de} \operatorname{dim}(c+z) \times 1
\end{aligned}
$$$$
\left(L-B_{x}^{\prime} K^{-1} B_{x}\right)=\lambda_{1}
$$$$
\left(Z-B_{X}^{\prime} K^{-1} \underset{\sim}{B}\right)=\lambda_{2} \text {, onde } \lambda_{1} \text { e } \lambda_{2} \text { são escalares; }
$$

obtēm-se :

$$
C_{\sim}^{0}+Q_{x} \hat{\gamma}=\underline{Q}
$$




$$
Q_{x}^{1} \stackrel{T}{1}_{1}^{0}+\lambda_{1} \bar{\gamma}=\lambda_{2}
$$

que $\bar{e}$ o sistema de equações normais livre dos efeitos de blocos.

Os escalares $B_{X}^{\prime} K^{-1} B_{X}$ e $B_{X}^{\prime} K^{-1}{ }_{B}$ são constituídos pela soma de quadrados e de produtos de blocos relativa a variā vel auxiliar e pela soma de produtos das variáveis auxiliar e dependente relativas a blocos, respectivamente.

Como vimos anteriormente, o vetor $\underline{Q} \bar{e}$ constituí do pelos totais de tratamentos, ajustados para blocos, relativos à variāvel dependente, os quais serão representados por $Q_{j .}(j=1,2, \ldots, c+z)$.

Os elementos da matriz $Q_{x}$ são semelhantes aos do vetor $\underline{Q}$, porém em relação à variāvel auxiliar, sendo que serão representados por $Q_{j l}(j=1,2, \ldots, c+z)$.

Uma outra maneira de chegar-se a (3.2.3.e) e (3.2.3.f) è prē-multiplicando o sistema (3.2.2.a) pela matriz

$$
W=\left[\begin{array}{l}
W_{1} \\
W_{2}
\end{array}\right]=\left[\begin{array}{ccc}
-N^{-1} & I(c+z) & \emptyset \\
-B_{x}^{\prime} K^{-1} & \emptyset & I(1)
\end{array}\right]
$$

obtendo-se o sistema

$$
w X^{\prime} X \theta^{0}=w X^{\prime} \underline{Y}
$$

Podem-se comprovar, entre outras, as seguintes

relações: 


$$
\begin{aligned}
& w_{1} X^{\prime} X w_{1}^{\prime}=c \\
& w_{1} X^{\prime} X w_{2}^{\prime}=Q_{X} \\
& w_{2} X^{\prime} X w_{2}^{\prime}=\lambda_{1} \\
& w_{1} X^{\prime} X \theta^{0}=C_{\sim}^{0}+Q_{X} \bar{\gamma} \\
& w_{2} X^{\prime} X \theta^{0}=Q_{X \sim 1}^{\prime}{ }^{0}+\lambda_{1} \bar{\gamma} \\
& w_{1} X^{\prime} \underline{\sim}=Q_{\sim}^{Q} \\
& w_{2} X^{\prime} \underline{\sim}=\lambda_{2}
\end{aligned}
$$

Aqui, a matriz C é a mesma apresentada no item 3.1.4. De (3.2.3.e) e utilizando-se a mesma matriz de restrí ção A usada em (3.1.4.1.b), obtēm-se:

$$
(C+A) \underset{\sim}{T}{ }_{1}^{0}+Q_{X} \bar{\gamma}=\underline{Q}
$$

onde $C+A=M$ é uma matriz não singular. Logo, a solução do sís tema é dada por

$$
I_{\sim}^{0}=M^{-1} Q-M^{-1} Q_{X} \bar{\gamma}
$$

ou

$$
\stackrel{T}{T}_{\sim}^{0}=T_{0}^{0}-T_{X}^{0} \bar{\gamma}
$$


Portanto:

$$
t_{j}^{0}=t_{j 0}^{0}-t_{j 1}^{0} \hat{\gamma} \quad(j=1,2, \ldots, c+z) \quad(3.2 .3 . j)
$$

onde:

$t_{j}^{0}$ : estimativa do efeito do j-ésimo tratamento ajustado pa ra blocos e regressão;

$t_{j o}^{0}$ : estimativa do efeito do j-ēsimo tratamento ajustado pa ra blocos relativo à variāvel Y;

$t_{j 1}^{0}:$ semelhante $\bar{a} t_{j o}^{0}$, porém em relação à variāvel auxiliar $x_{i j}$

$\hat{\gamma}$ : estimativa do coeficiente da regressão linear, associa da $\bar{a}$ variāvel auxiliar $x_{i j}$

Substituindo-se o valor de $\stackrel{\mathrm{T}}{1}_{1}^{0},(3.2 .3 . \mathrm{i}) \mathrm{em}$ $(3.2 .3 . f)$, segue-se que:

$$
Q_{X}^{1}\left(\stackrel{T}{0}_{0}^{0}-T_{X}^{0} \hat{\gamma}\right)+\lambda_{1} \hat{Y}=\lambda_{2},
$$

ou ainda:

$$
\left(\lambda_{1}-Q_{x}^{1} T_{x}^{0}\right) \bar{\gamma}=\left(\lambda_{2}-Q_{x}^{1} \tau_{0}^{0}\right) \quad(3.2 .3 . k)
$$

Os escalares $Q_{x}^{1} T_{x}^{0}$ e $Q_{X}^{1}{ }_{0}^{0}$ são constituídos pela soma de quadrados e de produtos de tratamentos ajustada para blocos referente à variāvel auxiliar, portanto, $Q_{x}^{\prime}{ }_{x}^{0}=T_{x}^{0} Q_{x}$ e pela soma de produto da variável auxiliar com a variāvel dependente ajustada para bloco, relativa a tratamento, respectivamente. 


$$
Q_{X}^{1} \underset{\sim 0}{0}=T_{x}^{0} Q
$$

Considerando-se

$$
\begin{aligned}
& \left(\lambda_{1}-Q_{x}^{\prime}{ }_{x}^{0}\right)=\left(\lambda_{1}-T_{x}^{0} Q_{x}\right)=\lambda_{3} \\
& \left(\lambda_{2}-Q_{x}^{1} \underset{\sim}{T}{ }_{0}^{0}\right)=\left(\lambda_{2}-T_{x}^{0} \underset{\sim}{Q}\right)=\lambda_{4}
\end{aligned}
$$

logo, de (3.2.3.k), tem-se:

$$
\lambda_{3} \hat{\gamma}=\lambda_{4}
$$

Os escalares $\lambda_{3}$ e $\lambda_{4}$ são constituídos pela soma de quadrado e de produtos do resīduo, relativa à variável a xiliar e pela soma de produto da variável auxiliar com a varia vel dependente, relativa ao residuo, respectivamente.

Então:

$$
\bar{\gamma}=\lambda_{3}^{-1} \lambda_{4}
$$

3.2.4. Somas de quadrados

3.2.4.1. Soma de quadrados de parâmetros

A soma de quadrados de parâmetros, relativa ao modelo (3.2.2.a), SQ Par (1), é obtida por:

SQ $\operatorname{Par}(1)=\theta^{0^{\prime}} X^{\prime} \underset{\sim}{Y}$ 


$$
\begin{aligned}
& =\left[\begin{array}{ccc}
\beta^{\prime} & \underline{T}^{O^{\prime}} & \bar{\gamma}
\end{array}\right]\left[\begin{array}{c}
B \\
T \\
\tau \\
Z
\end{array}\right] \\
& =\underline{B}^{0^{\prime}} \underline{B}+\stackrel{T}{T}^{0^{\prime}} \underset{\sim}{T}+\bar{\gamma} Z
\end{aligned}
$$

De (3.2.3.a), tem-se:

$$
B^{0}=K^{-1} \underset{B}{B}-K^{-1} N \cdot \underset{\sim}{\sim} \underset{1}{0}-K^{-1} B_{X} \bar{\gamma}
$$

$\log 0$ :

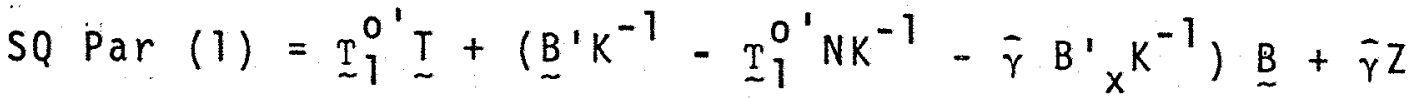

$$
\begin{aligned}
& ={\underset{\sim}{T}}_{1}^{0}\left(I-N K^{-1} \underset{\sim}{B}\right)+\underset{\sim}{B^{\prime}} K^{-1} \underset{\sim}{B}+\bar{\gamma}\left(Z-B_{X}^{\prime} K^{-1} \underset{\sim}{B}\right) \\
& =\stackrel{T}{T}_{1}^{0} \underline{Q}+\underline{\sim}^{\prime} K^{-1} \underset{\sim}{B}+\bar{\gamma} \cdot \lambda_{2}
\end{aligned}
$$

Substituindo-se (3.2.3.i) no primeiro termo da igualdade, vem:

$$
\begin{aligned}
& S Q \operatorname{Par}(1)=\left({\underset{\sim}{0}}_{0}^{0^{\prime}}-\bar{\gamma} T_{\mathrm{X}}^{0^{\prime}}\right) Q+\underline{B}^{\prime} \mathrm{K}^{-1} \underset{\sim}{\mathrm{B}}+\bar{\gamma} \lambda_{2}
\end{aligned}
$$

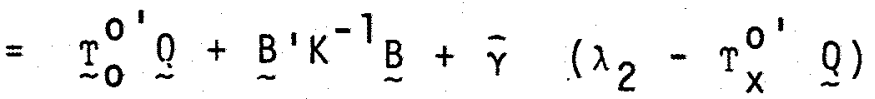

$$
\begin{aligned}
& ={\underset{\sim}{O}}_{0}^{0} \underset{\sim}{Q}+{\underset{\sim}{B}}^{\prime} K^{-1} \underset{\sim}{B}+\bar{\gamma} \cdot \lambda_{4} \text {, }
\end{aligned}
$$

onde: 
${ }_{\sim 0}^{O_{0}^{\prime}} Q=S Q T$ aj. $\quad$ soma de quadrados de tratamentos, ajustado para blocos, referente à variāvel dependente; $\underline{\sim}^{\prime} K^{-1} \underline{B}=S Q B \quad$ soma de quadrados de blocos, relativa à variā vel dependente;

$\hat{\gamma} \bar{\lambda}_{4}=$ SQReg soma de quadrados da regressão

3.2.4.2. Soma de quadrados do residuo, ajustada para regressão.

A soma de quadrados do residuo, ajustada para regressão, SQR* $\bar{e}$ obtida por:

$$
\begin{aligned}
& S Q R^{*}=\underline{Y}^{\prime} \underline{Y}-\operatorname{SQPar}(1) \\
& S Q^{*}=\left(\underline{Y}^{\prime} \underline{\sim}-\stackrel{T}{O}_{0}^{0^{\prime}} \underline{\sim}-\underline{\sim}^{\prime} K^{-1} \underset{\sim}{B}\right)-\bar{\gamma} \lambda 4 \\
& S Q R^{*}=R\left(Y^{2}\right)-\bar{\gamma} \lambda_{4}
\end{aligned}
$$

onde

$R\left(Y^{2}\right)$ é a soma de quadrados do residuo, sem serem considera das as variāveis auxiliares.

3.2.4.3. Soma de quadrados de tratamentos, ajus tada para blocos e regressão.

Seja o modelo matemático ao qual omitimos o 
efeito do tratamento

$$
Y_{i j}=\mu+\beta_{i}+\gamma x_{i j}+e_{i j}, \quad(3.2 .4 .3 . a)
$$

com $i=1,2, \ldots, b$ e $j=1,2, \ldots, c+z$,

onde: $Y_{i j}, \mu, \beta_{i}, \gamma, x_{i j}=X_{i j}-X$ e $e_{i j}$ tẽm significados semelhantes ao do modelo (3.2.1.a).

$$
\text { Usando-se a forma matricial, o modelo (3.2.4.3.a) }
$$

fica:

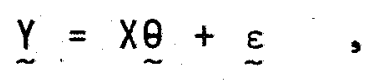

onde,

$\underline{y}$ é o vetor dos valores observados de $\operatorname{dim}(b c+z) \times 1$;

$X$ é a matriz dos coeficientes dos parāmetros, de rank b;

$\underline{\theta}$ è o vetor dos parâmetros;

$\underset{\varepsilon}{\varepsilon}$ o vetor dos erros experimentais, sendo que $\underset{\sim}{\varepsilon} \sim \mathrm{N}\left(0, \sigma^{2} \mathrm{I}\right)$.

Efetuando-se convenientemente a partição da matriz $x$ obtēm-se

$$
x=\left[\begin{array}{l:l}
x_{1} & x_{3}
\end{array}\right] \text {, }
$$$$
(3 \cdot 2 \cdot 4 \cdot 3 \cdot c)
$$

onde,

$x_{1}$ é a matriz dos coeficientes dos efeitos de biocos, de $\operatorname{dim}(b c+z) \times b$; 
$x_{3}$ é o vetor dos valores observados da variāvel auxiliar.

A partição do vetor $\theta$, correspondente a partição da matriz $x \bar{e}$

onde

$$
\stackrel{\sim}{\theta}^{\prime}=\left[\begin{array}{l:l}
\beta^{\prime} & \gamma \\
\hdashline & \gamma
\end{array}\right]
$$

$\underline{\beta}^{\prime}$ e $\gamma$ tem significados semelhantes ao do modelo (3.2.1.b) e com aquelas dimensões, respectivamente.

O sistema equações normais, obtido atravēs do método dos quadrados mínimos, é dado por:

$$
x^{\prime} \times \underline{\sim}^{0}=x^{\prime} \underline{\sim}
$$

ou seja,

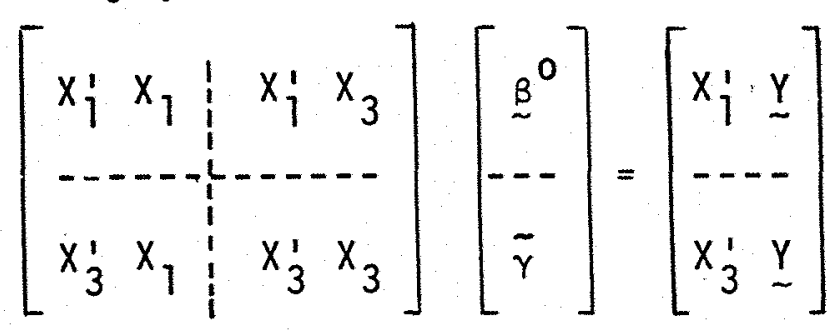

onde $x_{1}^{1} x_{1}, x_{1}^{1} x_{3}, x_{3}^{1} x_{1}$ e $x_{3}^{1} x_{3}$ tem significados semelhantes ao do modeto (3.2.2.b) 
escrito na forma:

$\left[\begin{array}{cc}K & B_{X} \\ B_{X}^{\prime} & L\end{array}\right]\left[\begin{array}{c}\tilde{\beta}^{0} \\ \cdot \\ \tilde{\gamma}\end{array}\right]=\left[\begin{array}{c}B \\ Z \\ Z\end{array}\right]$

$(3.2 .4 .3 . f)$

onde ${\underset{\beta}{\beta}}^{0}, \vec{\gamma}, \underset{\sim}{B}$ e $Z$ tem significados semelhantes ao do sís tema (3.2.2.c)

$$
0 \text { sistema }(3.2 .4 .3 . f) \text { resulta nas seguintes }
$$

equações

$$
\begin{aligned}
& \mathrm{K}_{\sim}^{0}+\mathrm{B}_{\mathrm{X}} \underset{\hat{\gamma}}{ }=\underset{\sim}{B} \\
& \mathrm{~B}^{\prime}{ }_{\mathrm{X}} \underline{\beta}^{0}+\mathrm{L} \tilde{\gamma}=Z
\end{aligned}
$$

Prē-multiplicando-se $(3 \cdot 2 \cdot 4 \cdot 3 . g)$ por $\left(-B_{x}^{\prime} K^{-1}\right)$ e somando-se em $(3.2 .4 .3 . h)$, tem-se:

$$
\begin{array}{ll}
\left(L-B_{X}^{\prime} K^{-1} B_{X}\right) \tilde{\gamma}=\left(Z-B_{x}^{\prime} K^{-1}{ }_{\sim}\right), & \text { ou por }(3 \cdot 2.3 . d): \\
\lambda_{1} \tilde{\gamma}=\lambda_{2} & (3 \cdot 2 \cdot 4 \cdot 3 \cdot i) \\
\tilde{\gamma}=\lambda_{1}^{-1} \cdot \lambda_{2} & (3)
\end{array}
$$

A soma de quadrados de parāmetros, relativa ao modelo (3.2.4.3.a), SQ Par (2) é obtida por:

$$
\text { SQ Par (2) }=\theta^{0^{\prime}} X^{\prime} Y
$$




$$
\begin{aligned}
& =\left[\begin{array}{ll}
\underline{B}^{0^{\prime}} & \tilde{\gamma}
\end{array}\right]\left[\begin{array}{l}
\underset{\sim}{Z} \\
Z
\end{array}\right] \\
& =\underline{B}^{0^{\prime}} \underset{\sim}{B}+\tilde{\gamma} Z
\end{aligned}
$$

De $(3.2 .4 .3 . g)$

$$
\underline{\sim}^{0}=K^{-1} B-K^{-1} B X^{\tilde{\gamma}}
$$

logo:

$$
\begin{aligned}
& S Q \operatorname{Par}(2)=\left({\underset{\sim}{B}}^{\prime} K^{-1}-\tilde{\gamma} B_{X}^{\prime} K^{-1}\right) \underset{\sim}{B}+\tilde{\gamma} Z \\
& =\underline{B}^{\prime} K^{-1} \underset{\sim}{B}-\tilde{\gamma} B_{X}^{\prime} K^{-1} \underline{B}+\tilde{\gamma} Z \\
& =\underset{\sim}{B} \cdot K^{-1} \underset{\sim}{B}+\left(Z-B_{X}^{\prime} K^{-1} \underset{\sim}{B}\right) \tilde{\gamma} \\
& =\underline{B}^{\prime} K^{-1} \underline{B}+\lambda_{2} \tilde{\gamma}
\end{aligned}
$$

Finalmente, a soma de quadrados de tratamentos ajustada para blocos e regressão, SQ T $T_{a j}^{*}$ e

$$
\begin{aligned}
\text { SQ } T_{a j .}^{*} & =S Q \operatorname{Par}(1)-S Q \operatorname{Par}(2) \\
& ={\underset{\sim}{0}}_{0}^{0^{\prime}} \underset{\sim}{Q}+\underset{\sim}{B^{\prime}} K^{-1} \underset{\sim}{B}+\bar{\gamma} \lambda_{4}-\underset{\sim}{B} K^{-1} \underset{\sim}{B}-\tilde{\gamma} \cdot \lambda_{2} \\
& ={\underset{\sim}{0}}_{0}^{0^{\prime}} \underline{Q}+\bar{\gamma} \lambda_{4}-\bar{\gamma} \lambda_{2} \\
& =S Q T_{a j}+\bar{\gamma} \lambda_{4}-\tilde{\gamma} \lambda_{2}
\end{aligned}
$$

Somando-se e subtraindo-se $R\left(Y^{2}\right)$ ao segundo mem bro de $(3.2 .4 .3 .1)$, tem-se: 


$$
\begin{aligned}
S Q T_{a j .}^{*} & =\left[S Q T_{a j}+R\left(Y^{2}\right)\right]-\left[R\left(Y^{2}\right)-\bar{\gamma} \lambda_{4}\right]-\tilde{\gamma} \lambda_{2} \\
& =R^{\prime}\left(Y^{2}\right)-S Q R^{*}-\tilde{\gamma} \lambda_{2}(3.2 .4
\end{aligned}
$$

onde

$$
R^{\prime}\left(Y^{2}\right)=S Q \dot{T}_{a j}+R\left(Y^{2}\right)
$$

3.2.5. Esperanças matemāticas das somas de quadrados

3.2.5.1. Esperança matemātica de $\underline{T}_{0}^{0^{\prime}} \underline{Q}$. Sabe-se que:

$$
\underline{T}_{0}^{0^{\prime}} \underline{Q}=\underline{Q}^{\prime} M^{-1} \underline{Q}=\underline{Q}^{\prime} M^{-1} \underline{Q}
$$

Mas, de $(3.2 .3 . \mathrm{g}), \underline{Q}=\mathrm{W}_{1} X^{\prime} \underset{\sim}{\mathrm{Y}}$, então, consideran do-se o modelo (3.2.1.a) sem o efeito da média, como PIMENTEL GOMES (1967), segue-se que:

$$
\begin{aligned}
& E\left(\underset{\sim}{T_{0}^{0}} \stackrel{Q}{0^{\prime}}\right)=E\left[\underline{Y}^{\prime} X W_{1}^{\prime} M^{-1} W_{1} X^{\prime} \underline{Y}\right] \\
& =E\left[\left(\underline{\theta}^{\prime} X^{\prime}+\underline{\varepsilon}^{\prime}\right) X W_{1}^{\prime} M^{-1} W_{1} X^{\prime}(X \underline{\theta}+\underset{\varepsilon}{\varepsilon})\right] \\
& =\underset{\sim}{\theta} X^{\prime} X W_{1}^{\prime} M^{-1} W_{1} X^{\prime} X \underset{\sim}{\theta}+E\left(\underset{\varepsilon^{\prime} X W^{\prime}}{M^{-1} W_{1} X^{\prime} \varepsilon}\right)
\end{aligned}
$$

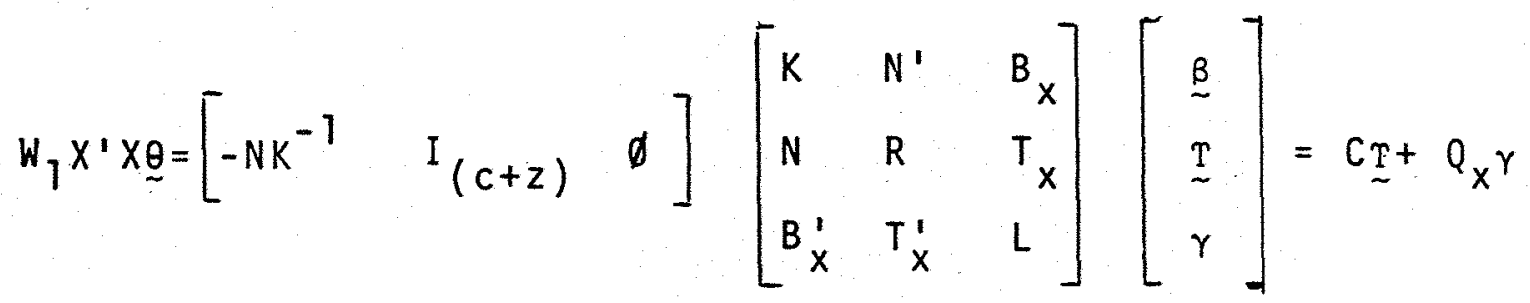

então 


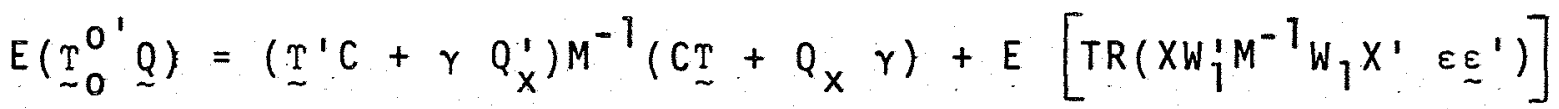

$$
\begin{aligned}
& =\left(\underline{\sim}^{\prime} C+\gamma Q_{X}^{\prime}\right) M^{-1}\left(\underset{\sim}{C}+Q_{X} Y\right)+T R\left[X W_{1}^{\prime} M^{-1} W_{1} X^{\prime} E\left(\underset{\varepsilon^{\prime}}{ }{ }^{\prime}\right)\right] \\
& =\left(\underline{I}^{\prime} C+\gamma Q_{X}^{\prime}\right) M^{-1}\left(C_{\underline{I}}+Q_{X} \gamma\right)+T R\left(M^{-1} W_{1} X^{\prime} X_{W}\right) \sigma^{2}
\end{aligned}
$$

Por $(3.2 .3 . g), W_{1} X^{\prime} X W_{1}^{\prime}=c$, tem-se:

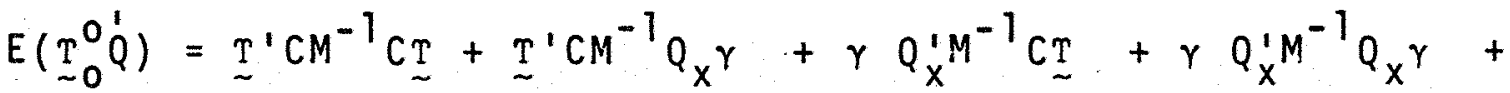

$$
\begin{aligned}
& \operatorname{TR}\left(M^{-1} C\right) \sigma^{2}
\end{aligned}
$$

por outro lado, IEMMA (1985) mostra çue a ma triz $M^{-1}$ è uma inversa condicional de $C$. Logo $C M^{-1} C=C$. Então $M^{-1} C$ e iderinotente, pois $M^{-1} C M^{-1} C=M^{-1} C$. e, portanto:

$$
\begin{aligned}
\operatorname{TR}\left(M^{-1} C\right) & =\operatorname{rank}\left(M^{-1} C\right) \\
& =\operatorname{rank}(C), \text { pois } M \text { é não-singular } \\
& =C+z-1
\end{aligned}
$$

Como $T_{x}=M^{-1} Q_{x}$ e $\gamma \bar{e}$ um escalar, logo,

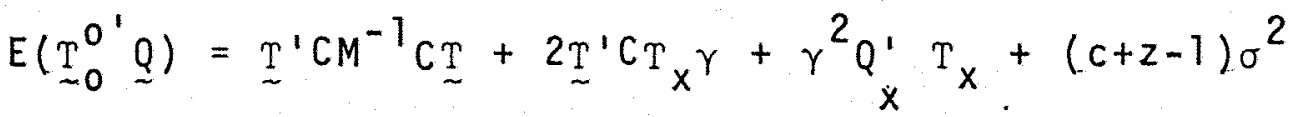

$$
\begin{aligned}
& (3.2 .5 \cdot 1 . a)
\end{aligned}
$$

Aqui, é conveniente mostrar que

$$
A M^{-1} W_{1} X^{\prime}=\varnothing
$$

$$
\text { Então } \begin{aligned}
A M^{-1} W_{1} X^{\prime} & =(C-M) M^{-1} W_{1} X^{\prime} \\
& =C M^{-1} W_{1} X^{\prime}-W_{1} X^{\prime}
\end{aligned}
$$


Pōs-multiplicando-se a expressão anterior por $X W_{1}^{\prime} M^{-1} A^{\prime}$ $A M^{-1} W_{1} X^{\prime} X W_{1}^{\prime} M^{-1} A^{\prime}=C M^{-1} W_{1} X^{\prime} X W_{1}^{\prime} M^{-1} A^{\prime}-W_{1} X^{\prime} X W_{1}^{\prime} M^{-1} A^{\prime}$

Por 3.2.3.g, $w_{1} X^{\prime} X w_{1}^{\prime}=c$, então:

$$
\begin{aligned}
A M^{-1} W_{1} X^{\prime} X W_{1}^{\prime} M^{-1} A^{\prime} & =C M^{-1} C M^{-1} A^{\prime}-C M^{-1} A^{\prime} \\
& =C M^{-1} A^{\prime}-C M^{-1} A^{\prime} \\
& =\varnothing, \text { ou seja. }
\end{aligned}
$$

$$
A M^{-1} W_{1} X^{\prime}=\varnothing
$$

Sendo $C=M-A, \quad \log 0$ :

$$
C M^{-1} \mathrm{C}=\mathrm{C}-\mathrm{AM}^{-1} \mathrm{C}
$$

e

$$
C T_{X}=C M^{-1} Q_{X}=Q_{X}=Q_{x}-A M^{-1} Q_{X}
$$

Pós-multiplicando-se 3.2.5.1.b, primeiramente por $X W_{1}^{\prime}$ e posteriormente por $X W_{2}^{\prime}$, obtēm-se:

$$
\begin{aligned}
& A M^{-1} W_{1} X \cdot X W_{1}^{1}=A M^{-1} C=\varnothing \\
& A M^{-1} W_{1} X ! X W_{2}^{\prime}=A M^{-1} Q_{X}=\varnothing
\end{aligned}
$$

respectivamente

$$
\begin{array}{lr}
\text { Assim, por } 3.2 .5 .1 . c \text { e } 3.2 .5 .1 . d \\
C M^{-1} C=c & (3.2 .5 .1 . \mathrm{h}) \\
C T_{X}=\mathrm{CM}^{-1} Q_{X}=Q_{X} & (3.2 .5 .1 . \mathrm{i})
\end{array}
$$


Finalmente

$$
\begin{array}{r}
E\left(T_{0}^{0} Q\right)=\underline{T}^{\prime} \underset{\sim}{T}+2 \underline{\sim}^{\prime} Q_{x} \gamma+\gamma^{2} Q_{x}^{\prime} T_{x}+(c+z-1) \sigma^{2} \\
(3.2 .5 .1 . j)
\end{array}
$$

3.2.5.2. Esperança matemātica da SQ Reg

$$
\begin{aligned}
& \operatorname{De}(3.2 .3 . \mathrm{m}) \text { e }(3.2 .3 .1) \text {, tem-se: } \\
& \dot{\gamma}=\lambda_{3}^{-1} \lambda_{4} \\
& \lambda_{4}=\lambda_{2}-r_{x}^{\prime} Q \quad \text { respectivamente. }
\end{aligned}
$$

Logo:

$$
\begin{gathered}
\bar{\gamma}=\lambda_{3}^{-1}\left(\lambda_{2}-T_{x}^{\prime} Q\right), \text { que nor }(3.2 .3 . g), \text { fica, } \\
\bar{\gamma}=\lambda_{3}^{-1}\left(W_{2} X^{\prime}-T_{X}^{\prime} W_{1} X^{\prime}\right) \underset{\sim}{Y} \quad(3.2 .5 .2 . a) \\
\bar{\gamma} \lambda_{4}=\underline{\sim}^{\prime}\left(X W_{2}^{\prime}-X W_{1}^{\prime} T_{X}\right) \lambda_{3}^{-1}\left(W_{2} X^{\prime}-T_{x}^{\prime} H_{1} X^{\prime}\right) \underline{\sim} \\
(3.2 .5 .2 . b)
\end{gathered}
$$

Assim:

$$
E\left(\bar{r} \lambda_{4}\right)=E\left[\underline{\gamma}^{\prime}\left(X W_{2}^{\prime}-X W_{1}^{\prime} r_{X}\right) \lambda_{3}^{-1}\left(W_{2} X^{\prime}-r_{X}^{\prime} W_{1} X^{\prime}\right) \underline{Y}\right]
$$




$$
\begin{aligned}
& =E\left[\left(\underline{\sim}^{\prime} X^{\prime}+\underset{\sim}{\varepsilon^{\prime}}\right)\left(X W_{2}^{\prime}-X W_{1}^{\prime} T_{X}\right) \lambda_{3}^{-1}\left(W_{2} X^{\prime}-T_{X}^{\prime} W_{1} X^{\prime}\right)(X \underset{\sim}{\theta}+\underset{\sim}{\varepsilon})\right] \\
& =\underline{\sim}^{\prime} X^{\prime}\left(X W_{2}^{\prime}-X W_{1}^{\prime} T_{X}\right) \lambda_{3}^{-1}\left(W_{2} X^{\prime}-T_{X}^{\prime} W_{1} X^{\prime}\right) X \underset{\sim}{\theta}+ \\
E & {\left[\underline{\sim}^{\prime}\left(X W_{2}^{\prime}-X W_{1}^{\prime} T_{X}\right) \lambda_{3}^{-1}\left(W_{2} X^{\prime}-T_{X}^{\prime} W_{1} X^{\prime}\right) \underset{\sim}{\varepsilon}\right] }
\end{aligned}
$$

Como

$$
W_{2} X^{\prime} X \theta=\left[\begin{array}{lll}
-B_{X}^{\prime} K^{-1} & \theta & I(1)
\end{array}\right]\left[\begin{array}{lll}
K & N^{\prime} & B_{X} \\
N & R & T_{X} \\
B_{X}^{\prime} & T_{X}^{\prime} & L
\end{array}\right]\left[\begin{array}{c}
\underset{B}{\sim} \\
\underset{\sim}{\gamma} \\
\gamma
\end{array}\right]=Q_{X}^{\prime} \underset{\sim}{T}+\lambda_{Y} \gamma
$$

e

$$
W_{1} X^{\prime} X \underline{\theta}=\underset{\sim}{c}+Q_{X} \gamma \quad \text { tem-se }
$$

$\left(W_{2} X^{\prime}-T_{X} W_{1} X^{\prime}\right) X \underset{\sim}{\theta}=Q_{X}^{\prime}{ }_{\sim}^{T}+\lambda_{1} \gamma-T_{X}^{\prime}{ }^{C}-T_{X}^{\prime} Q_{X} \gamma$

Como $\left(\lambda_{1}-T_{x}^{\prime} Q_{x}\right)=\lambda_{3}$ por $(3.2 .3 . k)$ e

$C T_{x}=Q x$ por $(3.2 .5 .1 . i)$, fica:

$$
\left(W_{2} X^{\prime}-T_{x}^{\prime} W_{1} X^{\prime}\right) x_{\sim}^{\theta}=\lambda_{3} \gamma \quad(3.2 .5 .2 . c)
$$

Então

$$
\begin{aligned}
& E\left(\bar{\gamma} \lambda_{4}\right)=\gamma^{2} \lambda_{3}+\operatorname{TR}\left[\left(X W_{2}^{\prime}-X W_{1}^{\prime} T_{x}\right) \lambda_{3}^{-1}\left(W_{2} X^{\prime}-T_{X}^{\prime} W_{1} X^{\prime}\right) E\left(\underline{\varepsilon}^{\prime}\right)\right] \\
& E\left(\bar{\gamma} \lambda_{4}\right)=\gamma^{2} \lambda_{3}+\operatorname{TR}\left[\lambda_{3}^{-1}\left(W_{2} X^{\prime}-T_{X}^{\prime} W_{1} X^{\prime}\right)\left(X W_{2}^{\prime}-X W_{1}^{\prime} T_{X}\right)\right] \sigma^{2}
\end{aligned}
$$

Tem-se

$$
\begin{aligned}
& \left(W_{2} X^{\prime}-\dot{r}_{x}^{\prime} W_{1} X^{\prime}\right)\left(X W_{2}^{\prime}-X W_{1}^{\prime} T_{x}^{\prime}\right)=w_{2} X^{\prime} X W_{2}^{\prime}-W_{2} X^{\prime} X W_{1}^{\prime} T_{X}^{\prime} \\
& -T_{x}^{\prime} w_{1} X^{\prime} X W_{2}^{\prime}+T_{x}^{\prime} w_{1} X^{\prime} X w_{1}^{\prime} T_{x}^{\prime}
\end{aligned}
$$


$\operatorname{Por}(3.2 .3 . g)$, fica:

$\left(W_{2} X^{\prime}-T_{X}^{\prime} W_{1} X^{\prime}\right)\left(X W_{2}^{\prime}-X W_{1}^{\prime} T_{x}^{\prime}\right)=\lambda_{1}-Q_{X}^{\prime} T_{x}-T_{X}^{\prime} Q_{x}+T_{x}^{\prime} C T_{X}$

$=\left(\lambda_{1}-T_{x}^{\prime} Q_{x}\right)+\left(T_{x}^{\prime} c-Q_{x}^{\prime}\right) T_{x}$ que por $(3.2 .3 . k) \mathrm{e}$

$(3.2 \cdot 5 \cdot 1 . i)$, fica:

$$
\left(W_{2} X^{\prime}-T_{x}^{\prime} W_{1} X^{\prime}\right)\left(X W_{2}^{\prime}-X W_{1}^{\prime} T_{x}^{\prime}\right)=\lambda_{3} \quad(3.2 .5 .2 . d)
$$

Finalmente:

$$
\begin{aligned}
& E\left(\hat{\gamma} \lambda_{4}\right)=\gamma^{2} \lambda_{3}+\sigma^{2} T R\left[\lambda_{3}^{-1} \dot{\lambda}_{3}\right] \\
& E\left(\bar{\gamma} \lambda_{4}\right)=\gamma^{2} \lambda_{3}+\sigma^{2} \quad(3.2 .5 .2 . e)
\end{aligned}
$$

3.2.5.3. Esperança matemātica de $\tilde{\gamma}_{2}$

$$
\operatorname{De}(3.2 .4 .3 . i) \text { e }(3.2 .3 . g) \text {, têm-se: }
$$$$
\tilde{\gamma}=\lambda_{1}^{-1} \lambda_{2}
$$$$
\lambda_{2}=W_{2} X^{\prime} \underline{Y} \text {, respectivamente. }
$$

Logo:

$$
\begin{array}{ll}
\tilde{\gamma}=\lambda_{1}^{-1} W_{2} X^{\prime} \underline{Y} & (3.2 .5 .3 . \mathrm{a}) \\
\tilde{\gamma} \lambda_{2}=\underline{Y}^{\prime} X W_{2}^{\prime} \lambda_{1}^{-1} W_{2} X^{\prime} \underline{Y} & (3 \cdot 2 \cdot 5.3 . \mathrm{b})
\end{array}
$$

Assim:

$$
E\left(\tilde{\gamma} \lambda_{2}\right)=E\left(\underline{\sim}^{\prime} X W_{2}^{\prime} \lambda_{1}^{-1} W_{2} X^{\prime} \underline{Y}\right)
$$




$$
\begin{aligned}
& =E\left[\left(\underline{\theta}^{\prime} X^{\prime}+\varepsilon^{\prime}\right) X W_{2}^{\prime} \lambda_{1}^{-1} W_{2} X^{\prime}(X \underline{\theta}+\underline{\varepsilon})\right] \\
& =\underline{\theta}^{\prime} X^{\prime} X W_{2}^{\prime} \lambda_{1}^{-1} W_{2} X^{\prime} X \underline{\theta}+E\left(\varepsilon_{\sim}^{\prime} X W_{2}^{\prime} \lambda_{1}^{-1} W_{2} X^{\prime} \underline{\varepsilon}\right) \\
& \text { Como } \\
& W_{2} X^{\prime} X=\left[\begin{array}{lll}
-B_{X}^{\prime} K^{-1} & \emptyset & I(1)
\end{array}\right]\left[\begin{array}{ccc}
K & N^{\prime} & B_{x} \\
N & R & T_{x} \\
B_{x}^{\prime} & T_{x}^{\prime} & L
\end{array}\right]= \\
& =\left[\emptyset \quad T_{x}^{\prime}-B_{x}^{\prime} K^{-1} N^{\prime} \quad L-B_{x}^{\prime} K^{-1} B_{x}\right]=\left[\begin{array}{lll}
\emptyset & Q_{x}^{\prime} & \lambda_{1}
\end{array}\right]
\end{aligned}
$$

então

$$
\begin{aligned}
& \underline{\theta^{\prime}} X^{\prime} \times W_{2}^{\prime} \lambda_{1}^{-1} W_{2} X^{\prime} X \underline{\theta}=
\end{aligned}
$$

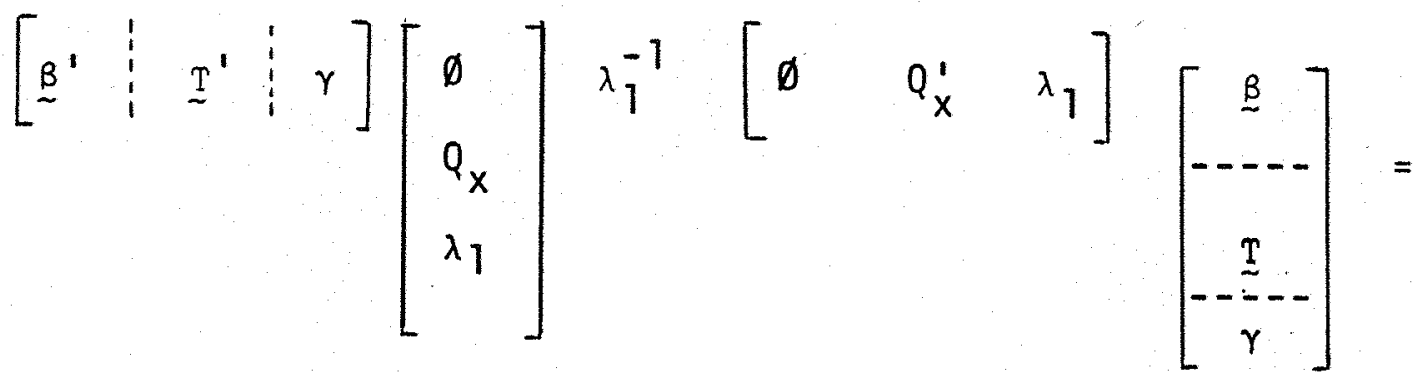

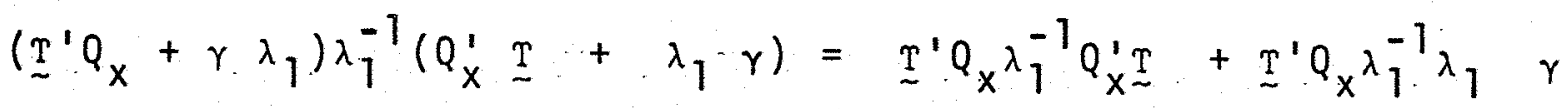

$$
\begin{aligned}
& +\gamma \lambda_{1} \lambda_{1}^{-1} Q_{X}^{\prime} T+\gamma \lambda_{1} \lambda_{1}^{-1} \lambda_{1} \gamma
\end{aligned}
$$

Portanto

$$
\begin{aligned}
& E\left(\tilde{\gamma} \lambda_{2}\right)=\underline{I}^{\prime} Q_{X} \lambda_{1}^{-1} Q_{X}^{\prime} \underline{\sim}+I_{\sim}^{\prime} Q_{X} \gamma+\gamma Q_{X}^{\prime} \stackrel{T}{\sim}+\gamma^{2} \lambda_{1}+E\left[T R\left(X W_{2}^{\prime} \lambda_{1}^{-1} W_{2} X^{\prime} \underline{\sim}_{\sim}^{\varepsilon^{\prime}}\right)\right]
\end{aligned}
$$

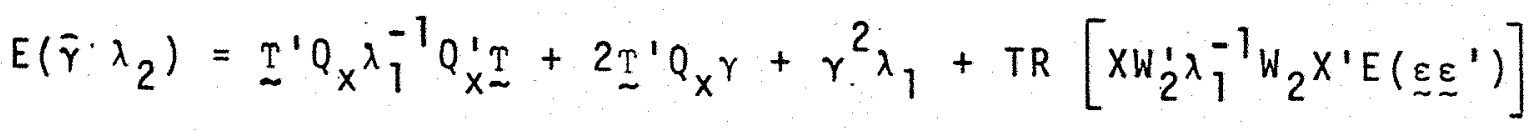




$$
\begin{aligned}
& E\left(\tilde{\gamma} \lambda_{2}\right)=I^{\prime} Q_{x} \lambda_{1}^{-1} Q_{X}^{\prime} I+2 T_{\sim}^{\prime} Q_{x}^{\gamma}+\gamma^{2} \lambda_{1}+T R\left(\lambda_{1}^{-1} W_{2} X^{\prime} X W_{2}^{\prime}\right) \sigma^{2} \\
& E\left(\tilde{\gamma} \lambda_{2}\right)=I^{\prime} Q_{X} \lambda_{1}^{-1} Q_{X}^{\prime} I+2 I_{\sim}^{\prime} Q_{X} \gamma+\gamma^{2} \lambda_{1}+T R\left(\lambda_{1}^{-1} \lambda_{1}\right) \sigma^{2}
\end{aligned}
$$

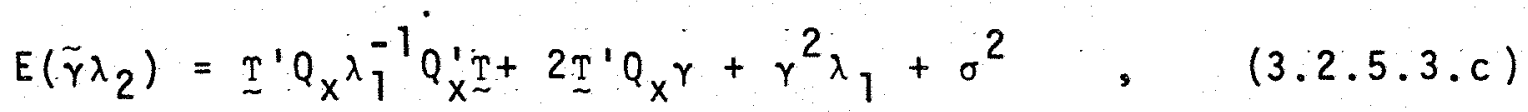

3.2.5.4. Esperança matemātica da SQT ${ }_{a j}^{*}$.

$$
\text { De }(3.2 .4 .3 . k) \text {, }
$$

$$
S Q T_{a j}^{*}=\stackrel{I}{0}_{0}^{0^{\prime}} Q+\bar{\gamma} \lambda_{4}-\tilde{\gamma} \lambda_{2}
$$

$$
E\left(S Q T_{a j}^{*}\right)=E\left(\underline{T}_{0}^{0^{\prime}} \underline{Q}\right)+E\left(\bar{\gamma} \lambda_{4}\right)-E\left(\tilde{\gamma} \lambda_{2}\right)
$$

Usando-se os resultados obtidos em $(3.2 .5 .1 . j)$ (3.2.5.2.e) e (3.2.5.3.c) tem-se, respectivamente:

$$
\begin{aligned}
& E\left(S Q T_{a j}^{*}\right)=\stackrel{T}{ }^{\prime} \mathbb{\sim}_{\sim}+2 \underline{\sim}^{\prime} Q_{x} \gamma+\gamma^{2} Q_{x}^{\prime}{ }^{T} x+(c+z-1) \sigma^{2}+\gamma^{2} \lambda_{3}+
\end{aligned}
$$

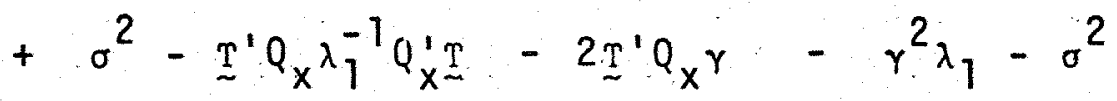

Como $\left(\lambda_{1}-Q_{x}^{T}{ }_{x}\right)=\lambda_{3}$, por $(3.2 .3: k)$, então

$$
E\left(S Q T_{a j .}^{*}\right)=\underline{T}^{\prime}\left(C-Q_{x} \lambda_{1}^{-1} Q_{x}^{\prime}\right) \underset{\sim}{T}+(c+z-1) \sigma^{2}
$$

3.2.5.5. Esperança matemātica de $\underline{Y}{ }^{\prime} \underline{Y}$ 


$$
\begin{aligned}
& \mathrm{SQT}=\underline{Y^{\prime}} \underline{Y} \\
& =\left(\underline{\sim}^{\prime} X^{\prime}+\underline{\varepsilon}^{\prime}\right)(X \underset{\sim}{\theta}+\varepsilon)
\end{aligned}
$$

Tem-se, então, que:

$$
\begin{aligned}
E(S Q T) & =\underline{\theta}^{\prime} X^{\prime} X \underset{\theta}{\theta}+E\left(\underline{\sim}^{\prime} \underline{\sim}\right) \\
& =\underline{\theta}^{\prime} X^{\prime} X \underline{\sim}+T R\left[E\left(\varepsilon^{\prime} \varepsilon\right)\right] \\
& =\underline{\sim}^{\prime} X^{\prime} X \underset{\theta}{\theta}+(b c+z) \sigma^{2}
\end{aligned}
$$

onde,

$$
\underline{\theta}^{\prime}=\left[\begin{array}{lll}
\underline{B}^{\prime} & \underline{T}^{\prime} & \gamma
\end{array}\right], \quad X^{\prime} X=\left[\begin{array}{ccc}
K & N^{\prime} & B_{X} \\
N & R & T_{X} \\
B_{X}^{\prime} & T_{X}^{\prime} & L
\end{array}\right]
$$

Assim,

$$
\begin{aligned}
& \underline{\theta}^{\prime} X^{\prime} X \underset{\sim}{\theta}=\left[\begin{array}{lll}
B^{\prime} & F^{\prime} & \gamma
\end{array}\right]\left[\begin{array}{ccc}
K & N^{\prime} & B_{X} \\
N & R & T_{X} \\
B_{X}^{\prime} & T_{X}^{\prime} & L
\end{array}\right]\left[\begin{array}{c}
\underline{B} \\
\mathcal{Z} \\
Y
\end{array}\right]= \\
& =\left(\underline{\beta}^{\prime} K+\underline{I}^{\prime} N+\gamma_{X}^{\prime}\right) \underset{\sim}{\beta}+\left(\beta^{\prime} N^{\prime}+\underline{\sim}^{\prime} R+\gamma_{X}^{\prime}\right) \underset{\sim}{T}+ \\
& +\left(\beta^{\prime} B_{X}+I^{\prime} T_{X}+\gamma L\right) \gamma \\
& =\underline{B}^{\prime} K_{\underline{B}}+\underline{T}^{\prime} N_{\underline{B}}+\gamma \underline{B}_{X}^{\prime} \underline{\underline{B}}+\underline{B}^{\prime} N^{\prime} \underline{\underline{T}}+\underline{T}^{\prime} R_{\mathcal{T}}+ \\
& +\gamma T_{X}^{\prime} I+\underline{\beta}^{\prime} B_{x} \gamma+I^{\prime} T_{x} \gamma+\gamma^{2} L
\end{aligned}
$$




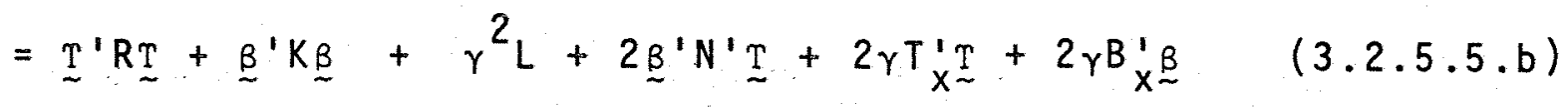

Substituindo-se $3.2 .5 .5 . b$ em $(3.2 .5 .5 . a)$, segue-se que:

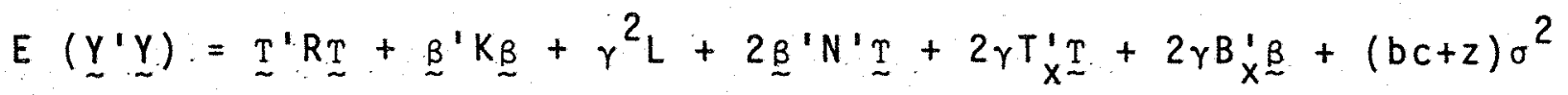

$(3.2 \cdot 5.5 . c)$

3:2.5.6. Esperança matemätica de $\underset{\sim}{B^{\prime}} K^{-1} \underset{\sim}{B}$

A soma de quadrados de blocos è dada por:

$S Q B=B^{\prime} K^{-1} \underset{\sim}{B}=\underline{\sim}^{\prime} X_{1} K^{-1} X_{i}^{\prime} \underset{\sim}{Y}$

Tem-se, então, que:

$$
\begin{aligned}
& E(S Q B)=E\left(\underline{Y}^{\prime} X_{1} K^{-1} X_{j}^{\prime} \underset{\sim}{Y}\right)=E\left[\left(\underline{\theta}^{\prime} X^{\prime}+\varepsilon^{\prime}\right) X_{1} K^{-1} X_{j}^{\prime}\left(X_{\sim}^{\theta}+\underline{\varepsilon}\right)\right] \\
& =\underline{\theta}^{\prime} X^{\prime} X_{1} K^{-1} X_{j} X_{\sim}^{\theta}+E\left(\varepsilon^{\prime} X_{1} K^{-1} X_{j} \varepsilon\right) \quad(3.2 .5 .6 . a)
\end{aligned}
$$$$
x^{\prime} x_{1}=\left[\begin{array}{c}
x_{1}^{\prime} \\
x_{2}^{\prime} \\
x_{3}^{\prime}
\end{array}\right] x_{1}=\left[\begin{array}{l}
K \\
N \\
B_{x}^{\prime}
\end{array}\right]
$$ 


$$
\begin{aligned}
& x^{\prime} x_{1} k^{-1} x_{1}^{\prime} x=\left[\begin{array}{c}
K \\
N \\
B_{x}^{\prime}
\end{array}\right] k^{-1}\left[\begin{array}{lll}
K & N^{\prime} & B_{x}
\end{array}\right] \\
& =\left[\begin{array}{ccc}
K & N^{\prime} & B_{X} \\
N & N K^{-1} N^{\prime} & N K^{-1} B_{X} \\
B_{X}^{\prime} & B_{X}^{\prime} K^{-1} N^{\prime} & B_{X}^{\prime} K^{-1} B_{X}
\end{array}\right]
\end{aligned}
$$

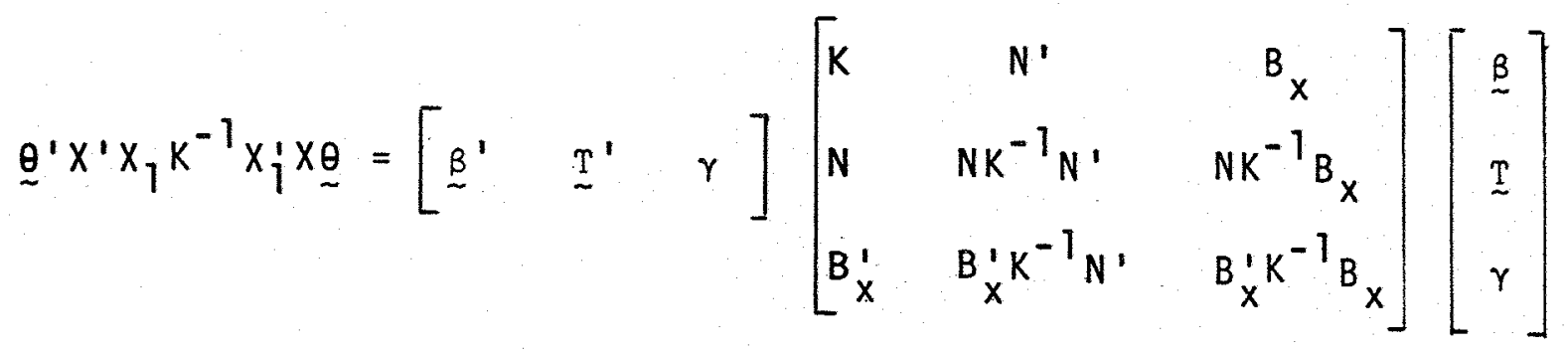

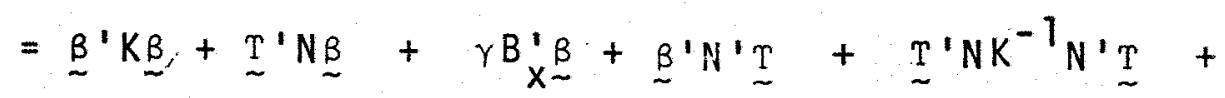

$$
\begin{aligned}
& +\gamma B_{X}^{\prime} K^{-1} N^{\prime} I+B^{\prime} B_{X}^{\gamma}+I^{\prime} N K^{-1} B_{X}^{\gamma}+\gamma^{2} B_{X}^{\prime} K^{-1} B_{X} \\
& =\underline{I}^{\prime} N K^{-1} N^{\prime} \underline{I}+\underline{B}^{\prime} K \underline{\beta}+\gamma^{2} B_{X}^{\prime} K^{-1} B_{X}+2 \underline{B}^{\prime} N^{\prime} \underline{T} \\
& +2 \gamma B_{X}^{\prime} K^{-1} N^{\prime} I+2 \gamma B_{X}^{\prime} B
\end{aligned}
$$

$$
\text { Substituindo-se }(3.2 .5 .6 . b) \text { em }(3.2 .5 .6 . a) \text {, }
$$
segue-se que:

$$
\begin{aligned}
E(S Q B)= & T^{\prime} N K^{-1} N^{\prime} \underline{T}+\underline{B^{\prime}} K_{\underline{B}}+\gamma^{2} B_{X}^{\prime} K^{-1} B_{X}+ \\
& +2 \underline{B}^{\prime} N^{\prime} \underline{T}+2 \gamma B_{X}^{\prime} K^{-1} N^{\prime} \underline{T}+2 \gamma B_{X}^{\prime} \underline{B}+ \\
& +T R\left[X_{1} K^{-1} X_{1}^{E}\left(\underline{\varepsilon} \underline{\sim}^{\prime}\right)\right]
\end{aligned}
$$




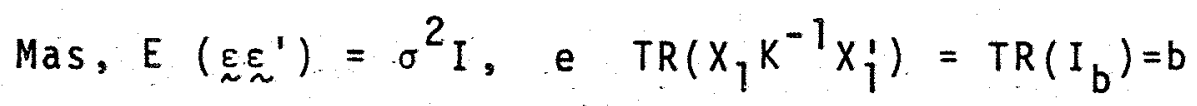

$\log 0$,

$$
\begin{aligned}
& E(S Q B)=\underline{I}^{\prime} N K^{-1} N^{\prime} \underline{I}+\underline{B}^{\prime} K \underline{B}+\gamma^{2} B_{X}^{\prime} K^{-1} B x+2 \underset{B^{\prime}}{ } N^{\prime} \underline{\sim}+ \\
& +2 \gamma B_{X}^{\prime} K^{-1} N^{\prime} \underset{\sim}{\mathcal{Z}}+2 \gamma B_{X}^{\prime} \underset{\sim}{B}+b \sigma^{2}
\end{aligned}
$$

3.2.5.7. Esperança matemātica da SQR*

De $(3.2 .4 .2 . a)$, tem-se:

$E\left(S Q R^{*}\right)=E\left({\underset{\sim}{Y}}^{\prime} \underset{\sim}{Y}\right)-E\left({\underset{\sim}{T}}_{0}^{0^{\prime}} \underset{Q}{Q}\right)-E\left(\underline{\sim}^{\prime} K^{-1} \underset{\sim}{B}\right)-E\left(\bar{\gamma} \lambda_{4}\right)$

Substituindo-se os resultados obtidos

em

$(3.2 .5 .5 . c),(3.2 .5 .1 . j),(3.2 .5 .6 . c)$ e $(3.2 .5 .2 . e)$ respec tivamente, tem-se, simplificando:

$$
\begin{aligned}
& E\left(S Q R^{*}\right)=I^{\prime} R \underset{\sim}{T}+\gamma^{2} L+2 \gamma T_{X \sim}^{\prime} \underset{\sim}{T}-\underset{\sim}{T} C \underset{\sim}{C}-
\end{aligned}
$$

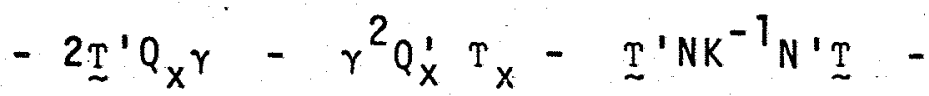

$$
\begin{aligned}
& -\gamma B_{X}^{\prime} K^{-1} B_{X} \gamma-2 \gamma B_{x}^{\prime} K^{-1} N \underset{\sim}{ }-\gamma^{2} \lambda_{3}+(b c-b-c) \sigma^{2}
\end{aligned}
$$

$$
\begin{aligned}
& E\left(S Q R^{*}\right)=\underset{\sim}{T^{\prime}}\left(R-N K^{-1} N^{\prime}\right) \underset{\sim}{T}+\gamma^{2}\left(L-B_{X}^{\prime} K^{-1} B_{X}\right) \\
& +2 \gamma\left(T{ }_{x}^{\prime}-B_{X}^{\prime} K^{-1} N^{\prime}\right) I-\underline{\sim}^{\prime} C \underline{\sim}-2 T^{\prime} Q_{X}^{\gamma} \\
& -\gamma Q_{x}^{1} x^{\gamma}-\gamma^{2} \lambda_{3}+(b c-b-c) \sigma^{2}
\end{aligned}
$$




$$
\begin{aligned}
& \text { Usando-se }(3 \cdot 2 \cdot 3 . d) \text {, tem-se: } \\
& E\left(S Q R^{*}\right)=I_{\sim}^{\prime} C_{\sim}+\gamma^{2} \lambda_{1}+2 \gamma Q_{X \sim}^{\prime}-I_{\sim}^{\prime} C_{I} \\
& -2 \sim^{\prime} Q_{x} \gamma-\gamma^{2} Q_{x}^{\prime} T_{x}-\gamma^{2} \lambda_{3}+(b c-b-c) \sigma^{2} \\
& =\gamma^{2}\left(\lambda_{1}-Q_{x}^{\prime}{ }_{x}\right)-\gamma^{2} \lambda_{3}+(b c-b-c) \sigma^{2} \\
& \operatorname{Como}\left(\lambda_{1}-Q_{x}^{\prime} T_{x}\right)=\lambda_{3},(3.2 .3 . k), \text { logo: } \\
& E\left(S Q R^{*}\right)=(b c-b-c) \sigma^{2}
\end{aligned}
$$

3.2.6. Distribuição das Formas Quadrāticas

Para determinar-se a distribuição das somas de quadrados apresentadas em (3.2), os seguintes teoremas foram utilizados

\section{TEOREMA 1 (GRAYBILL , 1961)}

Se $\underline{\sim} \sim N\left(\underline{\mu}, \sigma^{2} I\right)$, então $Y_{\sim}^{\prime} A Y / \sigma^{2} \approx X_{(n, \delta)}^{2}$, ou

ou seja, Y'AY/ ${ }^{2}$ tem distribuição de qui-quadrado com $n$ graus $1 i$ berdade e parāmetro de não centralidade

$$
\delta=\underline{\mu}^{\prime} A_{\underline{\mu}} / 2 \sigma^{2}
$$

se e somente se A for uma matriz idempotente de característica $n$. 


\section{TEOREMA 2 (SEARLE, 1971)}

Quando $\underset{\sim}{\mathrm{Y}} \sim \mathrm{N}(\underset{\sim}{\mu}, V)$, as formas quadrāticas Y'AY e Y ${ }^{\prime} B \underline{\sim}$ são distribuidas independentemente, se e somente se $A V B=\emptyset$, ou, de forma equivalente, $B V A=\emptyset$

\section{TEOREMA 3 (GRAYBILL, 1961)}

Sejam $W$ e $Z$ variāveis aleatōrias independentes tais que $w-x_{\left(n_{1}, \delta\right)}^{2}$ e $z-x_{n_{2}}^{2}$. Então,

$$
U=\frac{W / n_{1}}{Z / n_{2}} \text { tem distribuição } F \text {, não central com } n_{1} \text { e }
$$

$n_{2}$ graus de liberdade, e parāmetros de não centralidade $\delta$.

$$
\begin{aligned}
& \text { 3.2.6.1. Distribuição de }{\underset{\sim}{0}}_{0}^{0} Q \% \sigma^{2} \\
& \text { Sabe-se que SQT aj. } \bar{e}: \\
& \underline{T}_{0}^{O^{\prime}} Q=\underline{Q}^{\prime} M^{-1}{ }^{\prime} Q=Q^{\prime} M^{-1} \underline{Q}=\underline{Y}^{\prime} A_{1} \underline{Y}
\end{aligned}
$$

\section{Então}

$$
A_{1}^{2}=X W_{1}^{\prime} M^{-1} W_{1} X^{\prime} X W_{1} M^{-1} W_{1} X^{\prime}
$$


Mas, por $(3.2 .3 . g)$, chega-se a $w_{1} \times{ }^{\prime} X w_{1}^{\prime}=C$;

$\log 0$,

$$
\begin{aligned}
A_{1}^{2} & =X W_{1} M^{-1} \subset M^{-1} W_{1} X^{\prime} \\
& =X W_{1} M^{-1}(-A+M) M^{-1} W_{1} X^{\prime} \\
& =-X W_{1} M^{-1} A M^{-1} W_{1} X^{\prime}+X W_{1} M^{-1} W_{1} X^{\prime}
\end{aligned}
$$

que, por $(3.2 .5 .1 . c)$, fica:

$$
A_{1}^{2}=X W_{1} \cdot M^{-1} W_{1} X^{\prime}=A_{1}
$$

ou seja, $A_{1} \bar{e}$ uma matriz idempotente, e ainda,

$$
\text { Caract. } \begin{aligned}
\left(A_{1}\right) & =\operatorname{TR}\left(X W_{1}^{\prime} M^{-1} W_{1} X^{\prime}\right) \\
& =\operatorname{TR}\left(M^{-1} W_{1} X^{\prime} X W_{1}^{\prime}\right) \\
& =\operatorname{TR}\left(M^{-1} C\right) \\
& =\operatorname{TR}(C)=c+z-1
\end{aligned}
$$

Tem-se a inda:

$$
\underline{\mu}^{\prime} A_{1} \mu \underline{\theta}^{\prime} \times X^{\prime} \times w_{1}^{\prime} M^{-1} W_{1} X^{\prime} \times \underset{\sim}{\theta}
$$

que por $W_{1} X^{\prime} X \theta=C_{T}+Q_{X} \gamma,(3.2 .5 .1 . h)$ e $(3.2 .5 .1 . i)$ fica:

$$
\underline{\sim}^{\prime} A_{1} \underline{\sim}=\underset{\sim}{T}{ }^{C} \underset{\sim}{T}+2 T^{\prime} Q_{X} \gamma+\gamma^{2} Q_{X}^{\prime} T
$$


Assim, pelo Teorema 1, tem-se:

$$
{\underset{T}{0}}_{0}^{0} Q / \sigma^{2} \sim X_{\left(n_{1}, \delta_{1}\right)}^{2}
$$

onde

$$
\begin{aligned}
& n_{1}=c+z-1 ; \\
& \delta_{1}=\frac{1}{2 \sigma^{2}}\left(I^{\prime} C_{I}+2 \underline{T}^{\prime} Q_{X} \gamma+\gamma^{2} Q_{X}^{\prime}{ }^{T} X\right)
\end{aligned}
$$

3.2.6.2. Distribuição da SQ Reg/o

De $(3.2 .5 .2 . b)$, tem-se:

$$
\bar{\gamma} \lambda_{4}=\underline{Y}^{\prime}\left(X W_{2}^{\prime}-X W_{1}^{\prime} T_{X}\right) \lambda_{3}^{-1}\left(W_{2} X^{\prime}-T_{X}^{\prime} W_{1} X^{\prime}\right) \underline{Y}
$$

$$
=\underline{Y}^{\prime} A_{2} \underline{Y}
$$

onde $A_{2}=\left(X W_{2}^{\prime}-X W_{1}^{\prime} T_{x}\right) \lambda_{3}^{-1}\left(W_{2} X^{\prime}-T_{X}^{\prime} W_{1} X^{\prime}\right)$

Pode-se verificar que

$$
\begin{gathered}
A_{2}^{2}=\left(X W_{2}^{\prime}-X W_{1}^{\prime}{ }_{x}\right) \lambda_{3}^{-1}\left(W_{2} X^{\prime}-T_{x}^{\prime} W_{1} X^{\prime}\right)\left(X W_{2}^{\prime}-X W_{1}^{\prime} T_{x}\right) \lambda_{3}^{-1} \\
\left(W_{2} X^{\prime}-T_{x}^{\prime} W_{1} X^{\prime}\right)
\end{gathered}
$$

Mas, por $(3.2 .5 .2 . d)$, tem-se: 


$$
A_{2}^{2}=\left(X W_{2}^{\prime}-X W_{1}^{\prime} T_{X}\right) \lambda_{3}^{-1}\left(W_{2} X^{\prime}-T_{X}^{\prime} W_{1} X^{\prime}\right)=A_{2} \quad(3.2 .6 .2 . b)
$$

ou seja, $A_{2}$ é uma matriz idempotente, e ainda,

$$
\text { Caract. } \begin{aligned}
\left(A_{2}\right) & =\operatorname{TR}\left[\left(X W_{2}^{\prime}-X W_{1}^{\prime} T_{x}\right) \lambda_{3}^{-1}\left(W_{2} X^{\prime}-T_{x}^{\prime} W_{1} X^{\prime}\right)\right] \\
& =\operatorname{TR}\left[\lambda_{3}^{-1}\left(W_{2} X^{\prime}-T_{x}^{\prime} W_{1} X^{\prime}\right)\left(X W_{2}^{\prime}-X W_{1}^{\prime} x^{\prime}\right)\right] \\
& =\operatorname{TR}\left(\lambda_{3}^{-1} \lambda_{3}\right) \\
& =\operatorname{TR}(1) \\
& =1
\end{aligned}
$$

Tem-se, ainda:

$$
\underline{\mu}^{\prime} A_{2} \underset{\sim}{\mu}=\theta^{\prime} X^{\prime}\left(X W_{2}^{\prime}-X W_{1}^{\prime} r_{x}\right) \lambda_{3}^{-1}\left(W_{2} X^{\prime}-{ }^{\prime}{ }_{x}^{\prime} W_{1} X^{\prime}\right) x \theta,
$$

que, por $(3.2 .5 .2 . c)$, fica:

$$
\underline{\mu}^{\prime} A_{2} \underline{\mu}=\gamma^{2} \lambda_{3}
$$

Assim, pelo Teorema 1, tem-se:

$$
\bar{\gamma} \lambda_{4} / \sigma^{2}=S Q R e g / \sigma^{2}-X_{\left(n_{2}, \delta_{2}\right)}^{2}(3.2 .6 .2 . e)
$$

onde

$$
\begin{aligned}
& n_{2}=1 ; \\
& \delta_{2}=\frac{1}{2 \sigma^{2}} \gamma^{2} \lambda_{3}
\end{aligned}
$$


3.2.6.3. Distribuição de $\bar{\gamma} \lambda_{2} / \sigma^{2}$

$\operatorname{De}(3.2 \cdot 5.3 . \mathrm{b})$

$\tilde{\gamma} \lambda_{2}=\underline{Y}^{\prime} X W_{2}^{\prime} \cdot \lambda_{1}^{-1} W_{2} X^{\prime} \underline{Y}=\underline{Y}^{\prime} A_{3} \underline{Y}$

onde $A_{3}=X W_{2}^{\prime} \lambda_{1}^{-1} W_{2} X^{\prime}$.

Verifica-se que:

$$
A_{3}^{2}=\left(X W_{2}^{\prime} \lambda_{1}^{-1} W_{2} X^{\prime}\right)\left(X W_{2}^{\prime} \lambda_{1}^{-1} W_{2} X^{\prime}\right), \text { mas }
$$

$w_{2} X^{\prime} X w_{2}^{\prime}=\lambda_{1}$ por $(3.2 .3 . g), 10 g 0:$

$$
A_{3}^{2}=X W_{2}^{\prime} \lambda_{1}^{-1} W_{2} X^{\prime}=A_{3}
$$

isto è, $A_{3} \bar{e}$ uma matriz idempotente. Então:

$$
\begin{aligned}
\operatorname{Caract.}\left(A_{3}\right) & =\operatorname{TR}\left(X W_{2}^{\prime} \lambda_{1}^{-1} W_{2} X^{\prime}\right) \\
& =\operatorname{TR}\left(\lambda_{1}^{-1} W_{2} X^{\prime} X W_{2}^{\prime}\right) \\
& =\operatorname{TR}\left(\lambda_{1}^{-1} \lambda_{1}\right) \\
& =\operatorname{TR}(1) \\
& =1
\end{aligned}
$$

Tem-se ainda: 


$$
\underline{\mu}^{\prime} A_{3} \underline{\mu}=\underline{\sim}^{\prime} X X^{\prime} X W_{2}^{\prime} \lambda_{1}^{-1} W_{2} X^{\prime} X \underset{\sim}{\theta}
$$

que por $W_{2} X^{\prime} X_{\sim}=Q_{X}^{\prime} T+\lambda_{1}^{\gamma}$, fica:

$$
\underline{\mu}^{\prime} A_{3} \underline{\mu}=I^{\prime} Q_{x} \lambda_{1}^{-1} Q_{x}^{\prime} I+2 I^{\prime} Q_{x} \gamma+\gamma^{2} \lambda_{1}
$$

Assim, pelo teorema 1 , tem-se:

$$
\tilde{r} \lambda_{2} / \sigma^{2} \sim x_{\left(n_{3}, \delta_{3}\right)}^{2}
$$

onde:

$$
\begin{aligned}
& n_{3}=1 \\
& \delta_{3}=\frac{1}{2 \sigma^{2}}\left(T^{\prime} Q_{x} \lambda_{1}^{-1} Q_{x}^{1} T+{ }^{2} T^{\prime} Q_{x} \gamma+\gamma^{2} \lambda_{1}\right)
\end{aligned}
$$

3.2.6.4. Distribuição da SQTaj. $/ \sigma^{2}$

Sabe-se que $S Q T_{a j}^{*} . \quad \bar{e}:$

$$
\mathrm{SQT}_{\mathrm{aj} .}^{*}={ }_{\sim}^{\mathrm{T}^{0}}{ }^{\prime} Q+\bar{\gamma} \lambda_{4}-\bar{\gamma} \lambda_{2} \text { que, por }
$$

(3.2.6.1.a), (3.2.6.2.a) e(3.2.6.3.a), fica:

$$
\text { SQT }_{a j}^{*}=\underset{\sim}{Y^{\prime}}\left(A_{1}+A_{2}-A_{3}\right) \underset{\sim}{Y}=\underline{\sim}^{\prime} A_{4} \underset{\sim}{Y}
$$

onde $A_{4}=A_{1}+A_{2}-A_{3}$

\section{Tem-se:}

$$
A_{4}^{2}=A_{1}^{2}+A_{2}^{2}+A_{3}^{2}+A_{1} A_{2}+A_{1} A_{3}+A_{2} A_{1}-A_{2} A_{3}-A_{3} A_{1}-A_{3} A_{2}
$$


Mas:

$$
\begin{aligned}
& A_{1} A_{2}=X W_{1}^{\prime} M^{-1} W_{1} X^{\prime}\left(X W_{2}^{\prime}-X W_{1}^{\prime} T_{x}\right) \lambda_{3}^{-1}\left(W_{2} X^{\prime}-T_{x}^{\prime} W_{1} X^{\prime}\right) \\
& \operatorname{Por}(3.2 .3 . g) \\
& W_{1} X^{\prime}\left(X W_{2}^{\prime}-X W_{1}^{\prime} T_{x}\right)=Q_{x}-C_{T} \text { que, por }
\end{aligned}
$$

$(3.2 .5 .1 . i)$, tem-se:

$$
w_{1} X^{\prime}\left(X w_{2}^{\prime}-X w_{1}^{\prime} T_{x}\right)=\emptyset
$$

Assim, $\operatorname{por}(3.2 .6 .4 . \mathrm{b})$, tem-se:

$$
A_{1} A_{2}=\emptyset
$$

Sendo $A_{2} A_{1}=\left(A_{1} A_{2}\right)^{\prime}, 1090$ :

$$
A_{2} A_{1}=\varnothing
$$

Agora:

$$
A_{1} A_{3}=x W_{1}^{\prime} M^{-1} W_{1} x^{\prime} X W_{2}^{1} \lambda_{1}^{-1} W_{2} X^{\prime} \text { que, por }
$$

$(3.2 .3 . g), f i c a:$

$$
\begin{aligned}
& A_{1} A_{3}=X W_{1}^{\prime} M^{-1} Q_{x}{ }^{-1} W_{2} X^{\prime} \\
& \text { Sendo } M^{-1} Q_{X}=T_{x}, 10 g 0: \\
& A_{1} A_{3}=X W_{1}{ }^{T}{ }_{x}{ }^{\lambda}{ }_{1}^{-1} W_{2} X^{\prime}
\end{aligned}
$$$$
(3.2 .6 .4 . \mathrm{e})
$$ 


$$
\begin{aligned}
& \text { Como } A_{3} A_{1}=\left(A_{1} A_{3}\right)^{\prime}, \text { logo: } \\
& A_{3} A_{1}=X W_{2}^{\prime} \lambda_{1}^{-1} T_{X}^{\prime} W_{1} X^{\prime} \quad(3.2 .6 .4 . f)
\end{aligned}
$$

Por outro lado, tem-se:

$$
A_{2} A_{3}=\left(X W_{2}^{\prime}-X W_{1}^{\prime}{ }^{T}\right) \lambda_{3}^{-1}\left(W_{2} X^{\prime}-T_{x}^{\prime} W_{1} X^{\prime}\right)\left(X W_{2}^{\prime} \lambda_{1}^{-1} W_{2} X^{\prime}\right)
$$

$\operatorname{Por}(3.2 .3 . \mathrm{g})$, vem:

$$
\left(W_{2} X^{\prime}-T_{x}^{\prime} W_{1} X^{\prime}\right) X W_{2}^{\prime}=\left(\lambda_{1}-T_{x}^{\prime} Q_{x}\right), \quad \text { que }
$$

por $(3.2 .3 .1)$, fica:

$$
\left(w_{2} x^{\prime}-r_{x}^{\prime} w_{1} x^{\prime}\right) x w_{2}^{\prime}=\lambda_{3} \quad(3.2 .6 .4 . g)
$$

Portanto:

$$
A_{2} A_{3}=\left(X W_{2}^{\prime}-X W_{1}^{\prime} T_{X}\right) \lambda_{1}^{-1} W_{2} X^{\prime}, \text { que por }
$$

$(3.2 .6 .3 . a)$ e $(3.2 .6 .4 . e)$, fica:

$$
\begin{gathered}
A_{2} A_{3}=A_{3}-A_{1} A_{3} \\
\text { Como } A_{3} A_{2}=\left(A_{2} A_{3}\right)^{\prime}, 1090: \\
A_{3} A_{2}=A_{3}^{\prime}-\left(A_{1} A_{3}\right)^{\prime}=A_{3}-A_{3} A_{1}
\end{gathered}
$$

Finalmente, tem-se:

$$
\begin{aligned}
A_{4}^{2} & =A_{1}+A_{2}+A_{3}+\emptyset-A_{2} A_{3}+\dot{\theta}-\left(A_{3}-A_{1} A_{3}\right)- \\
& -A_{3} A_{1}-\left(A_{3}-A_{3} A_{1}\right)
\end{aligned}
$$


Apōs as simplificações, obtēm-se:

$$
A_{4}^{2}=\left(A_{1}+A_{2}-A_{3}\right)=A_{4}, \quad(3.2 \cdot 6 \cdot 4 \cdot j)
$$

is to $\bar{e}, A_{4} \bar{e}$ uma matriz idempotente. Assim sendo, segue-se que:

Caract. $\left(A_{4}\right)=\operatorname{TR}\left(A_{4}\right)=\operatorname{TR}\left(A_{1}+A_{2}-A_{3}\right)$

$$
=\operatorname{TR}\left(A_{1}\right)+\operatorname{TR}\left(A_{2}\right)-\operatorname{TR}\left(A_{3}\right) \text {, que }
$$

por $(3.2 .6 .1 . c),(3.2 .6 .2 . c)$ e $(3.2 .6 .3 . c)$, fica:

$$
\begin{aligned}
& \text { Caract. }\left(A_{4}\right)=(c+z-1) \quad(3.2 .6 .4 . k) \\
& \operatorname{Por}(3.2 .6 .1 . d),(3.2 .6 .2 . d) \text { e }(3.2 .6 .3 . d),
\end{aligned}
$$

apōs as simplificações, tem-se:

$$
\begin{aligned}
\underline{\mu}^{\prime} A_{4} \underline{\mu}=\underline{\mu}^{\prime}\left(A_{1}+A_{2}-A_{3}\right) \underline{\mu}= & \underline{I}^{\prime}\left(C-Q_{x} \lambda_{1}^{-1} Q_{x}^{\prime}\right) \underline{I} . \\
& (3.2 .6 .4 .1)
\end{aligned}
$$

Assim, pelo Teorema 1, tem-se:

$$
\operatorname{SQT}_{a j .}^{*} / \sigma^{2}-x_{\left(n_{4}, \delta_{4}\right)}^{2}
$$

onde

$$
\begin{aligned}
& n_{4}=(c+z-1) ; \\
& \delta_{4}=\frac{i}{2 \sigma^{2}} I^{\prime}\left(c-Q_{x} \cdot \lambda_{1}^{-1} Q_{x}^{\prime}\right) \stackrel{T}{~}
\end{aligned}
$$


3.2.6.5. Distribuição de $\underset{Y^{\prime}}{Y} \underset{\sim}{ } / \sigma^{2}$

Tem-se:

$$
\underline{Y}^{\prime} \underline{Y}=\underline{Y}^{\prime} I \underline{Y}=\underline{Y}^{\prime} A_{5} \underline{Y}
$$

onde $A_{5}=I(b \cdot c+z)$

Como $A_{5} \bar{e}$ uma matriz idempotente, de caracterís tica $(b . c+z)$, então, pelo Teorema 1 e por $(3.2 .5 .5 . b)$, tem-se:

$$
\underline{y} \underline{y} / \sigma^{2}-x_{\left(n_{5}, \delta_{5}\right)}^{2}
$$

onde:

$$
\begin{gathered}
n_{5}=b \cdot c+z ; \\
\delta_{5}=\frac{1}{2 \sigma^{2}}\left(\underline{\sim}^{\prime} R_{\sim}^{T}+\underline{B}^{\prime} K_{\sim}^{\beta}+\gamma^{2} L+2 \underset{\sim}{\beta} N^{\prime} \underline{\sim}+\right. \\
2 \gamma T_{X}^{\prime} \underline{\sim}+2 \gamma\left(B_{X}^{\prime} \underline{B}\right)
\end{gathered}
$$

3.2.6.6. Distribuição de $\underset{\sim}{B} K^{-1} \underset{\sim}{B} / \sigma^{2}$

Sabe-se que:

$$
\begin{aligned}
S Q B= & \operatorname{B}^{\prime} K^{-1} \underset{\sim}{B}={\underset{\sim}{Y}}^{\prime} X_{1} K^{-1} X_{1}^{\prime} \underset{\sim}{Y}=\underset{Y^{\prime}}{ } A_{6} \underset{\sim}{Y},(3.2 .6 .6 . a) \text { onde } \\
& A_{6}=X_{1} K^{-1} X_{1}
\end{aligned}
$$




\section{Verifica-se que:}

$A_{6}^{2}=x_{1} K^{-1} x_{1} x_{1} K^{-1} x_{1}=x_{1} K^{-1} K K^{-1} x_{1}=x_{1} K^{-1} x_{1}=A_{6}$,

(3.2.6.6.b) , ou seja, $A_{6} \bar{e}$ uma matriz idempotente; e ainda:

$$
\text { Caract. } \begin{aligned}
\left(A_{6}\right) & =\operatorname{TR}\left(X_{1} K^{-1} x_{j}\right) \\
& =\operatorname{TR}\left(I_{b}\right) \\
& =b \quad(3.2 .6 .6 . c)
\end{aligned}
$$

Tem-se ainda:

$$
\underline{\mu}^{\prime} A_{6} \underset{\sim}{\mu}=\underline{\theta}^{\prime} X^{\prime} X_{1} K^{-1} X_{1}^{\prime} x_{\sim} \text {, que, por }
$$

$(3.2 \cdot 5 \cdot 6 . b), f i c a:$

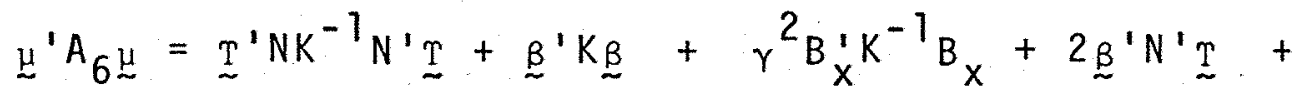

$$
\begin{aligned}
& +2 \gamma B_{X}^{\prime} K^{-1} N^{\prime} \underset{\sim}{T}+2 \gamma B_{X}^{\prime} \underset{\sim}{B}
\end{aligned}
$$

Assim, pelo Teorema 1, tem-se:

$$
\mathrm{SQB} / \sigma^{2} \sim \mathrm{X}_{\left(\mathrm{n}_{6}, \delta_{6}\right)}^{2}, \quad(3.2 \cdot 6.6 . \mathrm{e})
$$

onde,

$$
n_{6}=b
$$




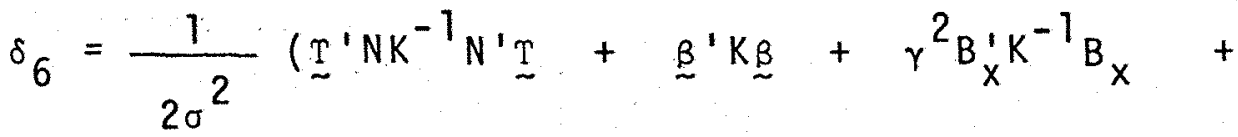

$$
\begin{aligned}
& \left.2 \underline{\sim}^{\prime} N^{\prime} \underset{\sim}{T}+2 \gamma B^{\prime} K^{-1} N^{\prime} \underset{\sim}{I}+2 \gamma B^{\prime} \underline{B}\right)
\end{aligned}
$$

3.2.6.7. Distribuiçã̃o de $S Q R^{*} / \sigma^{2}$

A SQR* é dada por:

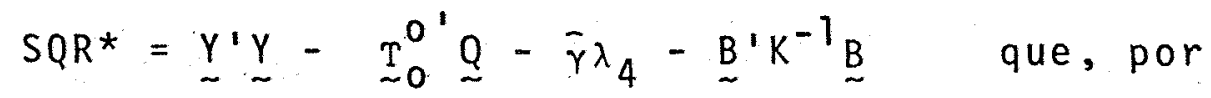

$(3.2 .6 .5 . a),(3.2 .6 .1 . a)(3.2 .6 .2 . a) \mathrm{e}(3.2 .6 .6 . a)$, fica:

$$
\begin{aligned}
& S R^{*}=Y^{\prime}\left(I-A_{1}-A_{2}-A_{6}\right) \underline{Y} \\
& =\underset{\sim}{Y} A_{7} Y
\end{aligned}
$$

onde $A_{7}=I-A_{1}-A_{2}-A_{6}$.

Tem-se:

$$
\begin{aligned}
A_{7}^{2} & =1^{2}+A_{1}^{2}+A_{2}^{2}+A_{6}^{2}-A_{1}-A_{2}-A_{6}-A_{1}+A_{1} A_{2}+A_{1} A_{6}- \\
& -A_{2}+A_{2} A_{1}+A_{2} A_{6}-A_{6}+A_{6} A_{1}+A_{6} A_{2}
\end{aligned}
$$

Vimos que $A_{1}, A_{2}$ e $A_{6}$ são matrizes idempotentes. $\operatorname{De}(3.2 .6 .4 . c)$ e $(3.2 .6 .4 . d)$, têm-se $A_{1} A_{2}=\emptyset$ e $A_{2} A_{1}=\phi$, respectivamente. 


$$
A_{1} A_{6}=x w_{1} M^{-1} W_{1} X^{\prime} x_{1} K^{-1} x_{1}
$$

onde $x^{\prime} x_{1}=\left[\begin{array}{l}x_{1}^{\prime} \\ x_{2}^{\prime} \\ x_{3}^{\prime}\end{array}\right] x_{1}=\left[\begin{array}{l}x_{1}^{\prime} x_{1} \\ x_{2}^{\prime} x_{1} \\ x_{3}^{\prime} x_{1}\end{array}\right]=\left[\begin{array}{c}k \\ N \\ B_{x}^{\prime}\end{array}\right]$

e

$W_{1} X^{\prime} X_{1}=\left[\begin{array}{ll}-N K^{-1} & I(c+z) \emptyset\end{array}\right]\left[\begin{array}{l}k \\ N \\ B_{X}^{\prime}\end{array}\right]=\emptyset, \quad(3 \cdot 2 \cdot 6.7 . b)$

Então, em (3.2.6.7.a), tem-se:

$A_{1} A_{6}=\theta$,

Como $A_{6} A_{1}=\left(A_{1} A_{6}\right)^{\prime}, \log 0$ :

$$
A_{6} A_{1}=\varnothing \quad
$$

Mas,

$$
W_{2} X^{\prime} X_{1}=\left[\begin{array}{lll}
-B_{X}^{\prime} K^{-1} & \emptyset & I(1)
\end{array}\right]\left[\begin{array}{l}
K \\
N \\
B_{x}^{\prime}
\end{array}\right]=\emptyset,(3.2 .6 .7 . e)
$$

Então,

$$
A_{2} A_{6}=\left(X W_{2}^{\prime}-x W_{1}^{\prime} T_{x}\right) \lambda_{3}^{-1}\left(W_{2} x^{\prime}-{ }_{x}^{\prime} w_{1} x^{\prime}\right) x_{1} k^{-1} x_{1}^{\prime}
$$


que por $3.2 .6 .7 . e$ e $3.2 .6 .7 . b$, fica:

$$
\begin{aligned}
& A_{2} A_{6}=\emptyset \\
& \text { Como } A_{6} A_{2}=\left(A_{2} A_{6}\right)^{\prime}, \text { logo: } \\
& A_{6} A_{2}=\emptyset,
\end{aligned}
$$

Finalmente, tem-se:

$$
\begin{aligned}
A_{7}^{2}= & I+A_{1}+A_{2}+A_{6}-A_{1}-A_{2}-A_{6}-A_{1}+0 \\
& +0-A_{2}+0+0-A_{6}+0+0 .
\end{aligned}
$$

Apōs as simplificações, ọbtēm-se:

$$
A_{7}^{2}=\left(I-A_{1}-A_{2}-A_{6}\right)=A_{7}, \quad(3.2 .6 .7 . h)
$$

isto $\bar{e}, A_{7}$ è uma matriz idempotente.

Assim sendo, segue-se que:

$$
\text { Caract. } \begin{aligned}
\left(A_{7}\right) & =\operatorname{TR}\left(I(b c+z)-A_{1}-A_{2}-A_{6}\right) \\
& =\operatorname{TR}\left(I(b c+z)-\operatorname{TR}\left(A_{1}\right)-\operatorname{TR}\left(A_{2}\right)-\operatorname{TR}\left(A_{6}\right)\right.
\end{aligned}
$$

Como $\operatorname{TR}(I(b c+z)=b c+z$ e usando-se $(3.2 \cdot 6 \cdot 1 . c)$, $(3.2 .6 .2 . c)$ e $(3.2 .6 .6 . c)$, tem-se: 
Caract. $\left(A_{7}\right)=(b \cdot c+z-c-z+\gamma-\gamma-b)$,

Caract. $\left(A_{7}\right)=(b c-c-b), \quad(3.2 .6 .7 . i)$

$\operatorname{Por}(3.2 .6 .5 . a),(3.2 .6 .1 . d),(3.2 .6 .2 . d) e$

(3.2.6.6.d), após todas as simplificações, obtēm-se:

$$
\mu^{\prime} A_{7} \mu=\mu^{\prime}\left(I-A_{1}-A_{2}-A_{6}\right) \mu=0,(3 \cdot 2 \cdot 6 \cdot 7 \cdot j)
$$

Assim, pelo Teorema 1, tem-se:

$$
S Q R^{*} / \sigma^{2}-X_{(n, 0)}^{2}, \quad(3.2 .6 .7 . k)
$$

ou seja, $S Q R^{*} / \sigma^{2}$ tem distribuição de qui-quadrado central, com $n_{7}$ graus de 1 iberdade, onde,

$$
n_{7}=(b c-b-c)
$$

3.2.6.8. Distribuição do quociente $\frac{\mathrm{SQReg} / \mathrm{n}_{2}}{S Q R^{*} / \mathrm{n}_{7}}$

De (3.2.6.2.a) e(3.2.6.7.a) , têm-se:

SQ Reg. $=\bar{\gamma} \lambda_{4}=\underline{Y}^{\prime} A_{2} Y$,

SQ Res* $=\underline{\sim}^{\prime}\left(I-A_{1}-A_{2}-A_{6}\right) \underset{\sim}{Y}=\underline{Y}^{\prime} A_{7} \underset{Y}{Y}$,

respectivamente. 
Verifica-se que, sendo $V=\sigma^{2} I$, então,

$$
\begin{aligned}
A_{2} V A_{7} & =A_{2} A_{7} \sigma^{2} \\
& =A_{2}\left(I-A_{1}-A_{2}-A_{6}\right) \sigma^{2} \\
& =\left(A_{2}-A_{2} A_{1}-A_{2}-A_{2} A_{6}\right) \sigma^{2} \\
& =\left(A_{2}-\emptyset-A_{2}-\emptyset\right) \sigma^{2} \\
& =\emptyset
\end{aligned}
$$

Assim, pelo Teorema 2, SQ Reg e SQR* são inde pendentes, e usando-se o Teorema 3 , tem-se, por (3.2.6.2.e) e $(3.2 .6 .7 . k)$, que:

$$
\frac{\text { SQ Reg./ } / n_{2}}{S Q R^{*} / n_{7}}-F\left(n_{2}, n_{7}, \delta_{2}\right)
$$

onde:

$$
\begin{aligned}
& n_{2}=1 ; \\
& n_{7}=(b c-b-c) ; \\
& \delta_{2}=\frac{1}{2 \sigma^{2}} \gamma^{2} \lambda_{3}
\end{aligned}
$$

3.2.6.9. Distribuição do quociente $\frac{S Q T_{a j . / n_{4}}^{*}}{S Q R^{*} / n_{7}}$

De 3.2.6.4.a e 3.2.6.7.a, tem-se: 


$$
\begin{aligned}
& \text { SQT } T_{a j .}^{*}=Y^{\prime}\left(A_{1}+A_{2}-A_{3}\right) \stackrel{Y}{\underline{L}}=Y^{\prime} A_{4} \underset{\sim}{Y}, \\
& S Q R^{*}=Y^{\prime}\left(I-A_{1}-A_{2}-A_{6}\right) \underset{\sim}{Y}=\underset{\sim}{Y^{\prime}} A_{7} \underset{\sim}{Y},
\end{aligned}
$$

respectivamente.

Verifica-se que, sendo $V=\sigma^{2} I$, então,

$$
\begin{aligned}
A_{4} V A_{7} & =A_{4} A_{7} \sigma^{2} \\
& =\left(A_{1}+A_{2}-A_{3}\right)\left(I-A_{1}-A_{2}-A_{6}\right) \sigma^{2} \\
& =\left[A_{1}-A_{1}^{2}-A_{1} A_{2}-A_{1} A_{6}+\right. \\
& +A_{2}\left(I-A_{1}-A_{2}-A_{6}\right)-A_{3}+A_{3} A_{1}+ \\
& \left.+A_{3} A_{2}+A_{3} A_{6}\right] \sigma^{2}, \quad(3.2 .6 .9 . a)
\end{aligned}
$$

Mas,

$$
A_{3} A_{6}=X W_{2}^{\prime} \lambda_{1}^{-1} W_{2} X^{\prime} x_{1} K^{-1} x_{1}^{\prime}, \text { que por(3.2.6.7.e), }
$$

fica: $\quad A_{3} A_{6}=\emptyset$,

Substituindo-se os valores obtidos em( $3.2 .6 .1 . b)$ $(3.2 .6 .4 . c),(3.2 .6 .7 . c),(3.2 .6 .8 . a),(3.2 .6 .4 . i) \quad e$ $(3.2 .6 .9 . b)$ em $(3.2 .6 .9 . a)$, e fazendo-se as simplificações, ob tēm-se : 


$$
\left(A_{1}+A_{2}-A_{3}\right)\left(I-A_{1}-A_{2}-A_{6}\right) \sigma^{2}=\emptyset,(3.2 .6 .9 . c)
$$

Assim, pelo Teorema 2, SQT aj. e SQR* são independentes, e usando-se o Teorema 3 , tem-se, por(3.2.6.4.m) e $(3.2 .6 .7 . k)$, que:

$$
\frac{\mathrm{SQT}_{a j}^{*} / n_{4}}{S Q R^{*} / n_{7}}-F_{\left(n_{4}, n_{7}, \delta_{4}\right)}
$$

onde:

$$
\begin{aligned}
& n_{4}=(c+z-1) ; \\
& n_{7}=(b c-b-c) ; \\
& \delta_{4}=\frac{1}{2 \sigma^{2}} \stackrel{T}{ }^{\prime}\left(c-Q_{x} \lambda_{1}^{-1} Q_{x}^{\prime}\right) \stackrel{\sim}{\sim}
\end{aligned}
$$

3.2.7. Estimadores das mëdias de tratamentos ajustadas para blocos e regressão.

As estimativas das médias dos tratamentos, ajus tudas para blocos e regressão, $\mu_{j}^{0}$, são obtidas por:

$$
\mu_{j}^{0}=\mu^{0}+t_{j}^{0} \quad(j=1,2, \ldots, c+z)
$$

onde $\mu^{0}=\frac{y}{b . c+z}$ 


$$
\mu_{j}^{0}=\mu_{j 0}^{0}-t_{j l} \bar{\gamma}(j=1,2, \ldots, c+z)(3.2 .7 . b)
$$

onde $\mu_{j o}^{0}=t_{j o}^{0}+\mu^{0}$ é a média de j-ésimo tratamento ajustado para blocos.

3.2.8. Quadro da añ̄i ise de covariância

Seguindo a notação de PIMENTEL GOMES (1985) temse o seguinte quadro da añ̄ise de covariāncia.

Quadro da anălise de covariancia

\begin{tabular}{|c|c|c|c|c|c|c|c|c|}
\hline \multirow{2}{*}{$\begin{array}{l}\text { Causa de } \\
\text { Variaçâo }\end{array}$} & \multirow[b]{2}{*}{ G.L. } & \multicolumn{3}{|c|}{ Somas de Quadrados e Produtos } & \multirow[b]{2}{*}{ G.L. } & \multirow[b]{2}{*}{ S.Q. } & \multirow[b]{2}{*}{$n . M$. } & \multirow[b]{2}{*}{$F$} \\
\hline & & $y^{2}$ & $x y$ & $x^{2}$ & & & & \\
\hline Blocos & $b-1$ & $B^{\prime} K^{-1} B-\frac{\left(\Sigma y^{2}\right)}{N}$ & $B_{x}^{\prime} K^{-1} B$ & $B_{x}^{\prime} K^{-1} B_{x}$ & & 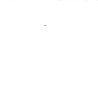 & & \\
\hline $\begin{array}{l}\text { Trat. (aj.p) } \\
\text { Blocos }\end{array}$ & $c+z-1$ & $I_{0}^{01} Q$ & $Q_{x}^{1} T_{0}^{0}=T_{x}^{0}{ }^{0}$ & $D_{x}^{1} T_{x}^{0}$ & & & & \\
\hline Residuo & $(b-1)(c-1)$ & $R\left(Y^{2}\right)$ & $x_{4}$ & $\lambda_{3}$ & $b c-b-c$ & $S Q R^{\star}$ & $\frac{S Q R^{*}}{b c-b-c}$ & \\
\hline Total & $b c+z-1$ & $\underline{Y} \underline{Y}-\frac{\left(\Sigma y^{2}\right)}{N}$ & $\begin{array}{rl}b & c+z \\
z & =\Sigma x_{i j} y_{i j}\end{array}$ & $L=\sum_{i j}^{b} \sum_{i j}^{c+7}$ & & & & \\
\hline $\begin{array}{l}\text { Trat.(aj. p/ } \\
\text { Blocos) + }\end{array}$ & $b(c-1)+z$ & $I_{0}^{0} Q+R\left(Y^{2}\right)$ & $\lambda_{2}$ & $\lambda_{1}$ & & & & \\
\hline Residuo & & & & & & & & \\
\hline Regressão & & & & & 1 & SQReg & $\frac{\text { SQReg }}{1}$ & $\frac{\text { SQReg/l }}{S O R * / b c-b-c}$ \\
\hline $\begin{array}{l}\text { Trat. (aj:p/ } \\
\text { Blocos e Regres } \\
\text { sao) }\end{array}$ & & & & & $c+2-1$ & $\operatorname{SQT}_{\mathrm{aj}}^{*}$ & $\frac{s+T_{a j} j}{c+z-1}$ & $\frac{S Q I^{*} a f / c+z-1}{S Q R^{*} / b c-b-c}$ \\
\hline
\end{tabular}


4. ILUSTRAÇAO DO METODO PROPOSTO

Considerando os dados hipotéticos, com 4 varie dades comuns e 7 variedades regulares, em 4 blocos

\begin{tabular}{|c|c|c|c|c|c|c|c|c|c|c|c|c|c|c|}
\hline \multirow{2}{*}{$\begin{array}{l}\text { Tra- } \\
\text { tamen } \\
\text { tos } \\
\end{array}$} & \multirow{2}{*}{$\begin{array}{r}\text { Blocos } \\
\text { varia- } \\
\text { veis }\end{array}$} & \multicolumn{3}{|c|}{$B_{1}$} & \multicolumn{3}{|c|}{$B_{2}$} & \multicolumn{3}{|c|}{$B_{3}$} & \multicolumn{3}{|c|}{$B_{4}$} & \multirow{2}{*}{ Total } \\
\hline & & $x_{i j}$ & $x_{i j}$ & $Y_{i j}$ & $x_{i j}$ & $x_{i j}$ & $Y_{i j}$ & $x_{i j}$ & $x_{i j}$ & $Y_{i j}$ & $x_{i j}$ & $x_{i j}$ & $Y_{i j}$ & \\
\hline & A & 3,00 & $(-0,40)$ & 1,61 & 2,50 & $(-0,90)$ & 2,30 & 5,00 & $(1,60)$ & 2,32 & 3,25 & $(-0,15)$ & 2,65 & 8,88 \\
\hline & B & 4,00 & $(0,60)$ & 3,73 & 3,75 & $(0,35)$ & 4,31 & 2,00 & $(-1,40)$ & 2,41 & 3,50 & $(0,10)$ & 3,21 & 13,66 \\
\hline & $c$ & 4,00 & $(0,60)$ & 1,12 & 4,00 & $(0,60)$ & 2,63 & 3,50 & $(0,10)$ & 0,56 & 3,75 & $(0,35)$ & 1,99 & 6,30 \\
\hline & $D$ & 3,00 & $(-0,40)$ & 1,55 & 4,00 & $(0,60)$ & 3,51 & 3,25 & $(-0,15)$ & 2,47 & 2,20 & $(-1,20)$ & 2,70 & 10,23 \\
\hline & e & 3,00 & $(-0,40)$ & 2,09 & & & & & & & & & & 2,09 \\
\hline & $f$ & 3,50 & $(0,10)$ & 2,87 & & & & & & & & & & 2,87 \\
\hline & $\mathbf{g}$ & & & & 3,00 & $(-0,40)$ & 1,47 & & & & & & & 1,47 \\
\hline & h & & & & 3,75 & $(.0,35)$ & 1,99 & & & & & & & 1,99 \\
\hline & $i$ & & & & & & & 4,50 & $(1,10)$ & 2,25 & & & & 2,25 \\
\hline & $\mathbf{j}$ & & & & & & & & & & 2,75 & $(-0,65)$ & 2,84 & 2,84 \\
\hline & $k$ & & & & & & & & & & 3,00 & $(-0,40)$ & 3,88 & 3,88 \\
\hline & $\Sigma$ & & & 12,97 & & & 16,21 & & & 10,01 & & & 17,27 & 56,46 \\
\hline
\end{tabular}


Pode-se então verificar que os parāmetros referentes ao delineamento em blocos completos aumentados, são:

$$
\begin{aligned}
& c=4, z=7, b=4, r_{j}=\left\{\begin{array}{l}
4, \text { para } j=1,2,3,4, \\
1, \text { para } j=5,6, \ldots, 11
\end{array}\right. \\
& k_{1}=6, k_{2}=6, k_{3}=5, k_{4}=6, N=23
\end{aligned}
$$

A matriz $x$ do delineamento de $\operatorname{dim}(23 \times 16)$

rank $15 \bar{e}$

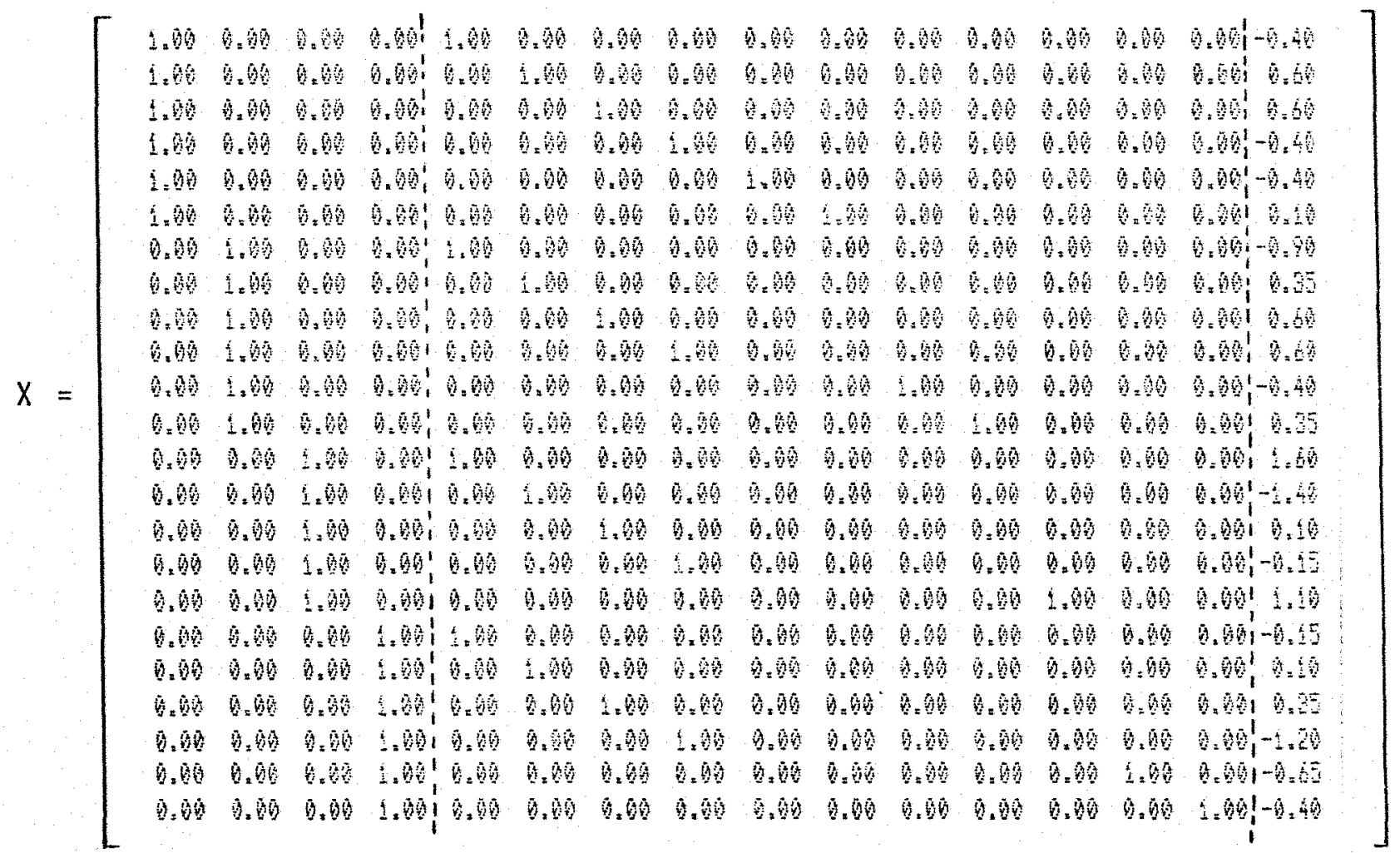


Assim, no sistema de equacões : $\because$ norma is $x^{\prime} x \underline{\sim}^{0}=x^{\prime} \underline{Y}$, tem $=$ se:

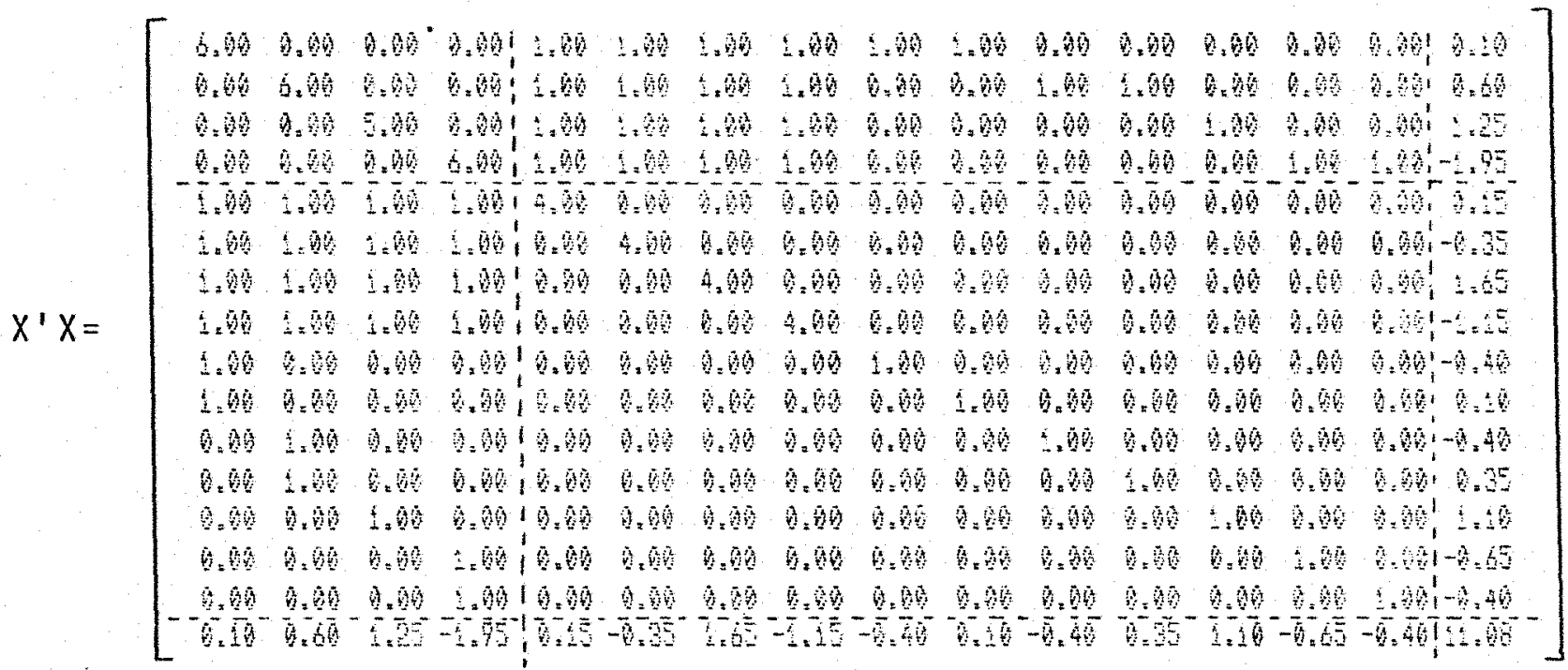

$=\left[\begin{array}{ccc}K & N^{\prime} & { }^{B} x \\ N & R & T_{X} \\ B_{X}^{\prime} & T_{X}^{\prime} & L\end{array}\right] ;$

$X: Y=\underline{\sim}=\left[\begin{array}{c}12,97 \\ 16,21 \\ 10,01 \\ 17,27\end{array}\right] ; X_{2}^{\prime} \underline{\sim}=\underline{\sim}=\left[\begin{array}{r}8,88 \\ 13,66 \\ 6,30 \\ 10,23 \\ 2,09 \\ 2,87 \\ 1,47 \\ 1,99 \\ 2,25 \\ 2,84 \\ 3,88\end{array}\right] ; X_{3}^{\prime} Y=Z=0,8085$ 
Para obter-se as estimativas dos efeitos dos parāmetros, obtēm-se, inicialmente as matrizes

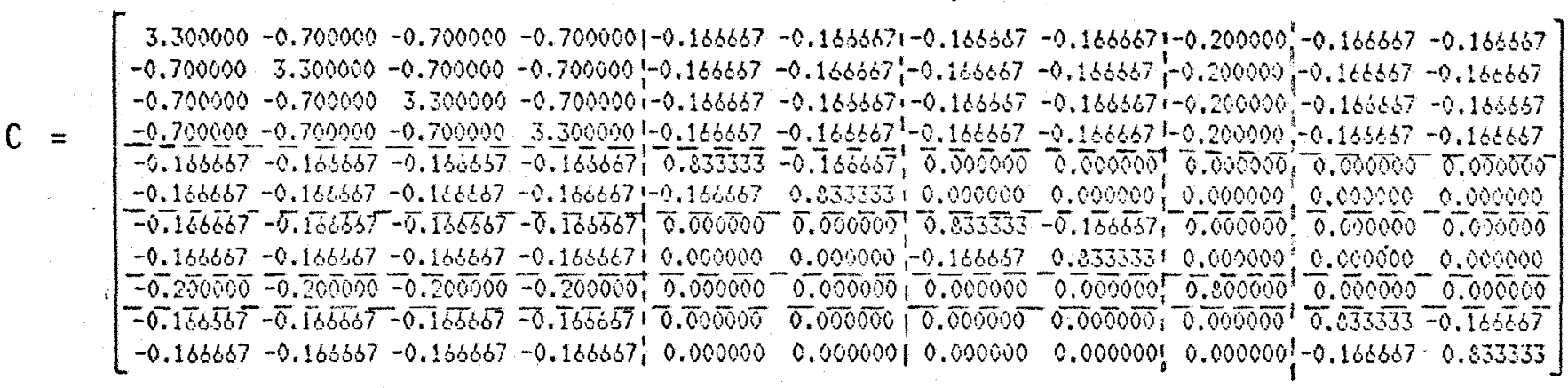

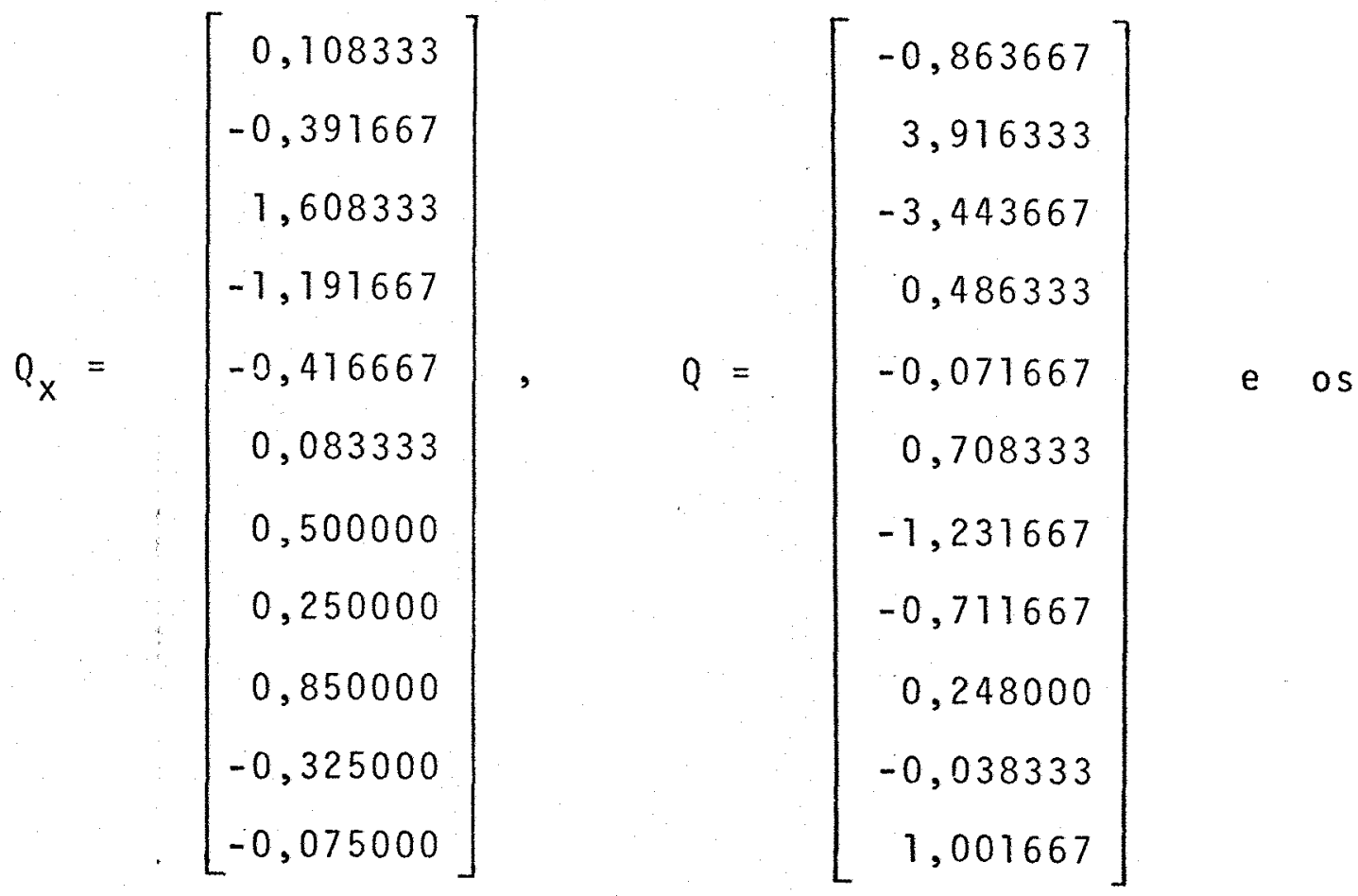

escalares $\lambda_{1}=10,077000$ e $\lambda_{2}=2,081583$.

Como $c$ e uma matriz singular, uma forma de contornar essa situação é mediante a introdução da matriz de res- 
trição A de rank 1 com as mesmas dimensões de $C$, isto $\bar{e}$, como dada no item 3.1 .4 .1 .

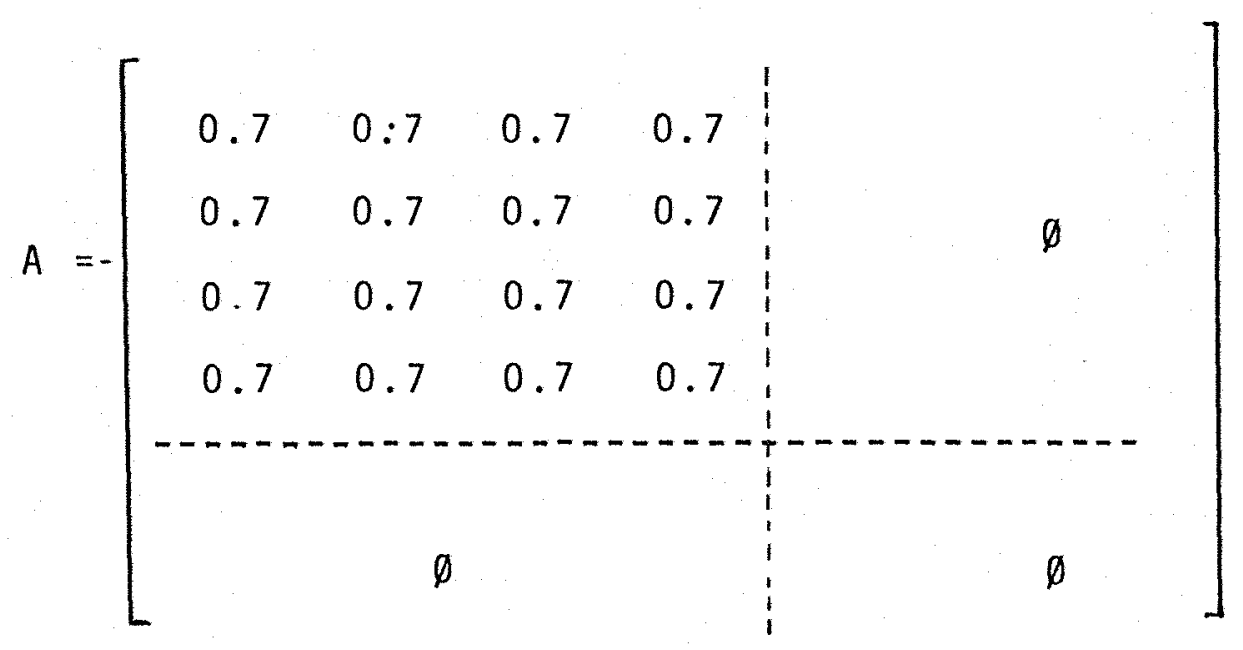

Portanto, $C+A=M \bar{e}$ uma matriz não singular onde, a matriz M de $\operatorname{dim}(11 \times 11) \overrightarrow{\mathrm{e}}$

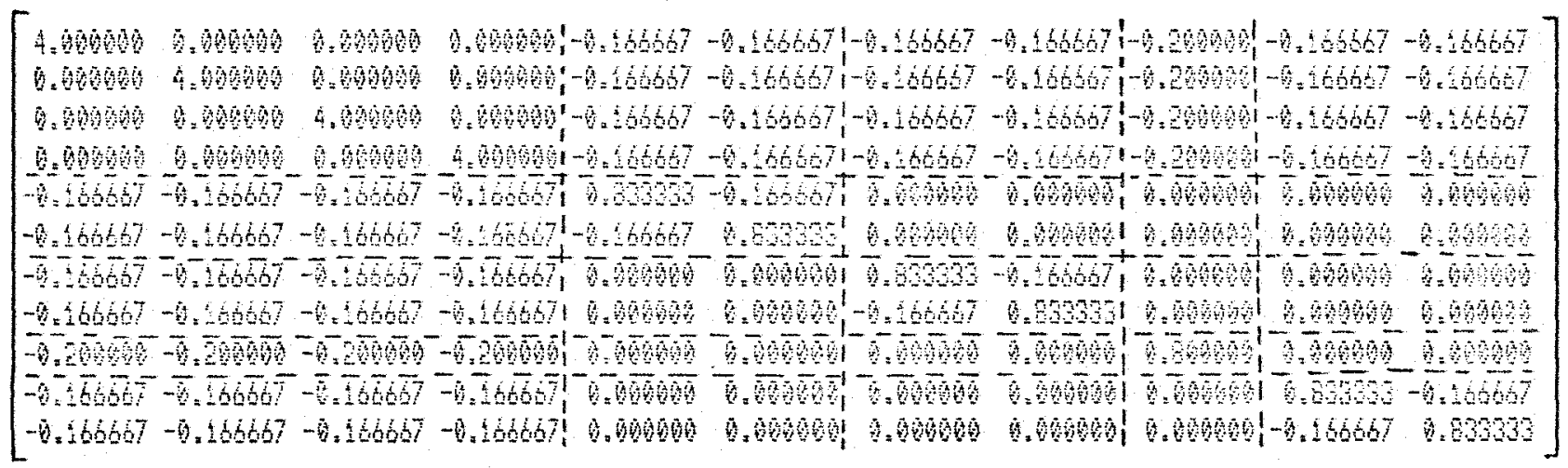

\section{e a $M^{-1} \vec{e}$}

\begin{tabular}{|c|c|c|c|c|c|c|}
\hline 0.276780 & 0.926750 & 0.06706 & nob7s, & 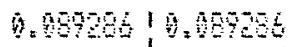 & 0.09986 1 609281 & 6 \\
\hline 0.92078 & 0.276786 & 0.90736 & 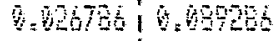 & 7. $90720,1,602 \mathrm{bo}$ & 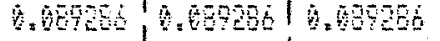 & 206 \\
\hline 0.92675 & 9.62076 & 578 & 1. 12576, 10925 & 4.98\%so 1 & $4,60906, i$ & \\
\hline 0.02078 & 0.09786 & $A=076$ & 4.2767 & 0.680 & 25 & \\
\hline 6,48980 & $8.00 \mathrm{~s}$ & 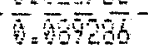 & 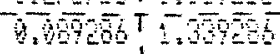 & $7.9606,13096$ & , & $7=$ \\
\hline 0.8 & $n_{z}$ & & 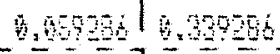 & & $-502 I^{4}$ & \\
\hline & & & 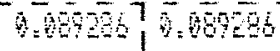 & 0.6908 & $030210,06020,3$ & \\
\hline 5.2 & 0.005206 & a & $\theta_{s}$ & 20013 & 8 & \\
\hline & & $\overline{9}, \overline{\mathrm{n}}$ & & 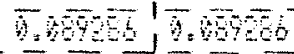 & 1.0906 & 5 \\
\hline 6. $4 \overline{\mathrm{DT}} \mathrm{E}$ & 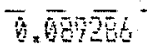 & inter & 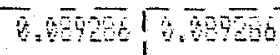 & 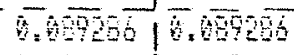 & 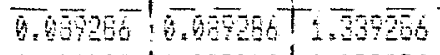 & 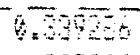 \\
\hline 8986 & 0.709206 & 6. 10706 & 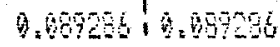 & 4.90206 9.96720 & 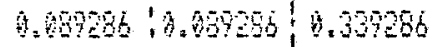 & \\
\hline
\end{tabular}




$$
\begin{aligned}
& \operatorname{Por}(3.2 .3 .1) \text {, obtēm-se } \\
& \lambda_{3}=7,444291 \text { e } \lambda_{4}=3,319062 .
\end{aligned}
$$

Assim a estimativa do coeficiente de regressão $\bar{e}$ :

$$
\bar{\gamma}=\lambda_{3}^{-1} \lambda_{4}=0,4459
$$

Como $\underset{\sim}{T}={ }_{\sim}^{0} \stackrel{T}{0}_{0}^{0}-T_{x}^{0} \hat{\gamma} \cdot \operatorname{por}(3.2 .3 . i)$, então o vetor das estimativas dos efeitos de tratamentos ajustados para blocos e regressão é:

$$
\sim_{1}^{0}=\left[\begin{array}{r}
-0,230236 \\
1,020502 \\
-1,042448 \\
0,252182 \\
0,310451 \\
0,867500 \\
-1,466683 \\
-1,281108 \\
-0,163769 \\
0,392009 \\
1,320534
\end{array}\right]=\left[\begin{array}{r}
-0,221875 \\
0,973125 \\
-0,866875 \\
0,115625 \\
0,087500 \\
0,867500 \\
-1,717502 \\
-1,197502 \\
0,310000 \\
0,202501 \\
1,242501
\end{array}\right]-\left[\begin{array}{r}
0,018750 \\
-0,106250 \\
0,393750 \\
-0,306250 \\
-0,500001 \\
0 \\
-0,562500 \\
0,187500 \\
1,062500 \\
-0,425001 \\
-0,175001
\end{array}\right] \cdot 0,4459
$$

$$
\begin{aligned}
& \text { Por (3.2.4.2.b), tem-se: } \\
& S Q R^{*}=R\left(Y^{2}\right)-\hat{\gamma} \lambda_{4} \quad \text { onde } \\
& R\left(Y^{2}\right)=\underset{\sim}{Y^{\prime}} \underset{\sim}{Y}-{\underset{\sim}{O}}_{0}^{0^{\prime}} \underline{Q}-\underline{\sim}^{\prime} K^{-1} \underset{\sim}{B}=156,355200-11,933678- \\
& -141,579670=2,841852
\end{aligned}
$$




$$
\begin{aligned}
& \bar{r}_{4}=(0,4459)(3,319062)=1,479970 \\
& S Q^{*}=2,841852-1,479970=1,361882
\end{aligned}
$$

A estimativa do coeficiente de regressão no mode10 $(3.2 .4 .3 . a) \bar{e} \tilde{\gamma}=\lambda^{-1} \lambda_{2}=(10,077)^{-1}(2,081583)$

$$
\begin{aligned}
& \tilde{r} \lambda_{2}=(0,206568) \cdot(2,081583)=0,429988 \\
& \text { Usando-se }(3.2 .4 .3 . \mathrm{m}) \text {, tem-se: } \\
& \begin{aligned}
S Q T_{a j}^{*}=R^{\prime}\left(Y^{2}\right)-S Q R^{*}- & \gamma \lambda_{2} \text { onde } \\
R^{\prime}\left(Y^{2}\right)=S Q T_{a j .}+R\left(Y^{2}\right) & =11,933678+2,841852 \\
& =14,775530
\end{aligned}
\end{aligned}
$$

Então a SQT ajj. $^{*}=14,775530-1,361882-0,429988$

$$
\mathrm{SQT}_{\mathrm{aj}}^{*}=12,983660
$$

Finalmente, o quadro da anālise de covariāncia dos dados fica 


Causa de
Variação

$\begin{array}{lllll}\text { Blocos } & 3^{\circ} & 2,982644 & -1,273083 & 1,007917\end{array}$

Trat.(aj.p/Blocos) $10 \quad 11,933678-1,237479 \quad 2,632709$

$\begin{array}{llllllll}\text { Resíduo } & 9 & 2,841852 & 3,319062 & 7,444291 & 8 & 1,361882 & 0,170235\end{array}$

$\begin{array}{lllll}\text { Total } & 22 & 17,758174 & 0,808500 & 11,084917\end{array}$

Trat. (aj.p/Blocos)

$\begin{array}{lllll}+ \text { Resîduo } & 19 & 14,775530 & 2,081583 & 10,077000\end{array}$

Regressão

$1 \quad 1,4799701,4799708,69 *$

Trat. (aj.p/BTocos

e Reg.)

Note-se que houve aumento de eficiēncia com $\quad 0$ uso da covariancia, pois o quadrado médio residual que : era 0,315761 , foi reduzido a 0,170235 .

As estimativas das mëdias de tratamentos ajustados para blocos, e para blocos e regressão, obtidas atravēs de ( $3.2 .7 . b)$, encontram-se na Tabela seguinte. 


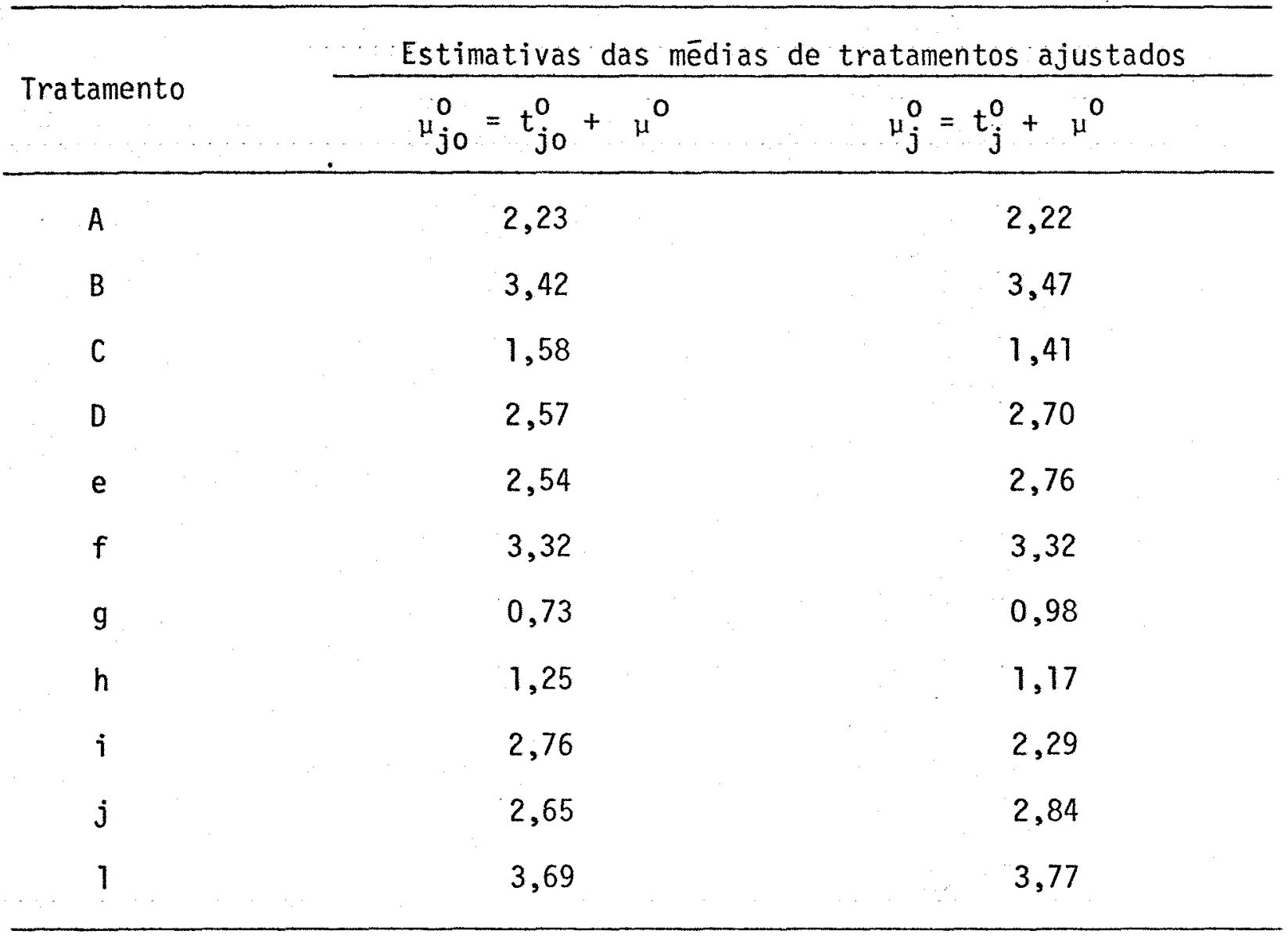

$\mu^{0}=2,45$ 
5. CONCLUSOES

Em geral, os resultados obtidos permitem as seguintes conclusões:

a) Quanto ao modelo matemático

0 modelo matemático apresentou-se adequado aos objetivos propostos.

b) Quanto à estimação dos efeitos dos tratamen tos

o método permitiu uma estimativa dos tratamentos condizentes com o objetivo.

c) Quanto à anāitise de covariāncia

0 modelo e o método permitiram obter-se os efei- 
tos de tratamentos ajustados para blocos e regressão levando o interessado a uma conclusão mais realista.

-d) Quanto aos testes de significância

A esperança matemātica das somas de quadrados mostraram a viabilidade do uso do teste $F$ na anālise de covariāncia.

e) Quanto ao exemplo numērico

o exemplo numērico mostrou a viabilidade da me todologia desenvolvida. 


\section{REFERENCIAS BIBLIOGRAFFICAS}

BACCHI, 0.0.S., 1985. Ecofisiologia da cana-de-açūcar. IAA/PLA NALSUCAR, Piracicaba. 20 p.

COCHRAN, W.G., 1957. Analysis of covariance: its nature and uses. Biometrics, Raleigh, 13: 261-281.

COX, D.R. e P. McCULLAGH, 1982. Some aspects of analys is of covariance (with discussion). Biometrics, Raleigh, 38: 541561 .

DIAS, C.T.S., 1985. Algebra matricial: um programa didático aplicado à estatistica. Seminārios de Estatistica e Experimentação Agronōmica, Piracicaba, ESALQ/USP, 20 semestre, $15 p$.

DIAS, J.F.S., 1981. Anālise de covariāncia intrablocos, com $\underline{p}$ variāveis auxiliares, para delineamentos em blocos incomple tos equilibrados. Piracicaba, ESALQ/USP, 96 p. (Tese de Doutoramento). 
FEDERER, W.T., 1956 a. Augmented (or Hoonuiaku) Designs. The Hawaitan Planter's Record, 55: 177-190.

FEDERER, W.T., 1956 b. Augmented (or Hoonuiaku) Designs : The Hawaitian Planter's Record, 55: 191-208

FEDERER, W.T., 1961. Augmented designs with one-way elimination of heterogeneity : Biometrics, Raleigh, 17: 447-473.

FEDERER, W.T. E D. RAGHAVARAO, 1975. On augmented designs. Biometrics, Raleigh, 31: 29-35.

GRAYBILL, F.A., 1961. An Introduction to Linear Statistical

Models. McGraw - Hill, Nova York. $463 \mathrm{p}$.

IEMMA, A.F., 1985. Modelos Lineares - Uma Introducão para Profissionais da Pesquisa Agropecuāria. ESALQ/USP, Piracicaba, 142 p. (10 SEAGRO).

KATTI, S.K., 1965. Multiple covariate analysis. Biometrics, Rialeigh, 21: 957-974.

KIRK, R.E., 1968. Experimental Designs: Procedures for Behavioral Sciences. Wadsworth, $577 \mathrm{p}$.

MARTINEZ GARZA, A., 1972. Diseño y Analisis de Experimentos 
con Caña de Azucar. Colegio de Postgraduados, Escuela Nacio nis de Agricultura, Chapingo, Mexico. 204 p.

NETER, J. e W. WASSERMAN, 1974. Applied Linear Statistical Models. Regression, Analysis of Variance and Experimental Designs. Irwin, $842 \mathrm{p}$.

NOGUEIRA, M.C.S., 1976. Anālise conjunta para experimentos em biocos casualizados completos aumentados. Piracicaba, ESALQ/ USP, 49 p. (Dissertação de Mestrado).

OGAWA, J., 1974: Statistical Theory of Analysis of Experimental Designs. Marcel Dekker in., Nova York. $465 \mathrm{p}$.

PAVATE, M.V., 1961. Combined analysis of balanced incomplete block designs with some common treatments. Biometrics, Raleigh, 17: 111-119.

PIMENTEL GOMES, F., 1967. The solution of normal equations of experiments in incomplete blocks. Ciência e Cultura, São Paulo, 20: 733-746.

PIMENTEL GOMES, F., 1985. Curso de Estatistica Experimental. 11 a ed., Livraria Nobel, Piracicaba. $466 \mathrm{p}$.

SEARLE, S.R., 1971. Linear Models. Willey, Nova York. 532 p. 
VIZONI, E., 1984. Anālise de experimentos em blocos casualizados completos aumentados (blocos de Federer), com parcelas subdivididas no tempo. Piraicaba, ESALQ/USP, 125 p. (Disser tação de Mes.trado).

ZELEN, M., 1957. The analysis of covariance for incomplete block designs. Biometrics, Raleigh, 13: 309-332. 